\title{
ANALYTIC EVOLUTION OF SINGULAR DISTRIBUTION AMPLITUDES IN QCD
}

by

\author{
Asli Tandogan \\ M.S. May 2008, Old Dominion University
}

A Dissertation Submitted to the Faculty of Old Dominion University in Partial Fulfillment of the Requirements for the Degree of

DOCTOR OF PHILOSOPHY

PHYSICS

OLD DOMINION UNIVERSITY

August 2014

Approved by:

Anatoly Radyushkin (Director)

Ian Balitsky (Member)

Gail Dodge (Member)

Alex Godunov (Member)

Oktay Baysal (Member) 


\title{
ABSTRACT \\ ANALYTIC EVOLUTION OF SINGULAR DISTRIBUTION AMPLITUDES IN QCD
}

\author{
Asli Tandogan \\ Old Dominion University, 2014 \\ Director: Dr. Anatoly Radyushkin
}

Distribution amplitudes (DAs) are the basic functions that contain information about the quark momentum. DAs are necessary to describe hard exclusive processes in quantum chromodynamics. We describe a method of analytic evolution of DAs that have singularities such as nonzero values at the end points of the support region, jumps at some points inside the support region and cusps. We illustrate the method by applying it to the evolution of a flat (constant) DA, antisymmetric flat DA, and then use the method for evolution of the two-photon generalized distribution amplitude. Our approach to DA evolution has advantages over the standard method of expansion in Gegenbauer polynomials [1, 2] and over a straightforward iteration of an initial distribution with evolution kernel. Expansion in Gegenbauer polynomials requires an infinite number of terms in order to accurately reproduce functions in the vicinity of singular points. Straightforward iteration of an initial distribution produces logarithmically divergent terms at each iteration. In our method the logarithmic singularities are summed from the start, which immediately produces a continuous curve. Afterwards, in order to get precise results, only one or two iterations are needed. 
Copyright, 2014, by Asli Tandogan, All Rights Reserved. 


\section{ACKNOWLEDGEMENTS}

I would like to express my great gratitude and deepest appreciation to my advisor Prof. Anatoly Radyushkin. Learning such a high level physics from him was an irreplaceable life time opportunity. His passion and dedication for physics inspired me to aim higher in life. His patience, wisdom and encouraging talks guided me through my intellectual improvement.

I warmly thank Prof. Ian Balitsky, Prof. Gail E. Dodge, Dr. Alexander L. Godunov and Prof. Oktay Baysal for valuable time they have dedicated for my education.

There are no words to describe the appreciation and gratitude I feel for my husband Mike. I would like to thank him for all support, consideration, encouragement and understanding. I am also very grateful to my family for being there for me unconditionally. I am very thankful that my son Çınar has joined our family in May 2013. He motivates me every day to improve myself personally and academically. 


\section{TABLE OF CONTENTS}

Page

LIST OF FIGURES $\ldots \ldots \ldots \ldots \ldots \ldots \ldots \ldots \ldots \ldots \ldots \ldots \ldots \ldots \ldots \ldots \ldots \ldots \ldots \ldots \ldots \ldots \ldots$

Chapter

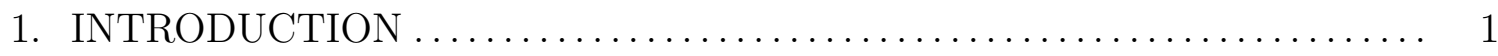

1.1 QUANTUM CHROMODYNAMICS $\ldots \ldots \ldots \ldots \ldots \ldots \ldots \ldots \ldots \ldots$

$1.2 \quad$ DEEP INELASTIC SCATTERING $\ldots \ldots \ldots \ldots \ldots \ldots \ldots \ldots \ldots \ldots$

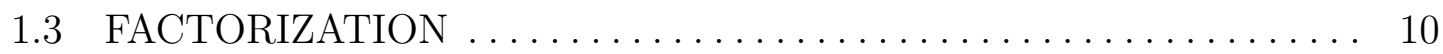

2. EVOLUTION OF SINGULAR DISTRIBUTION AMPLITUDES . . . . . . . . 12

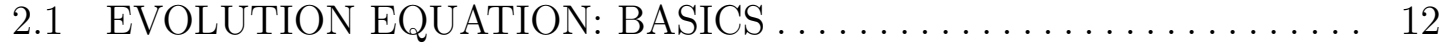

2.2 DISTRIBUTION AMPLITUDES (DA) AND THEIR EVOLUTION WITH GEGENBAUER EXPANSION: . . . . . . . . . . . . . . . . . . . 19

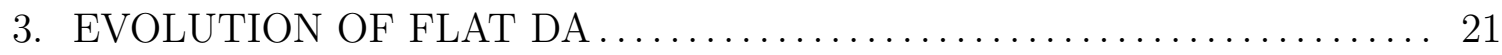

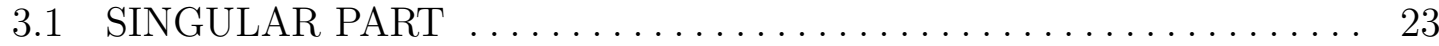

3.2 ADDING NON-SINGULAR PART $\ldots \ldots \ldots \ldots \ldots \ldots \ldots \ldots \ldots$

4. EVOLUTION OF ANTI-SYMMETRIC FLAT DA $\ldots \ldots \ldots \ldots \ldots \ldots \ldots \ldots . . \ldots$

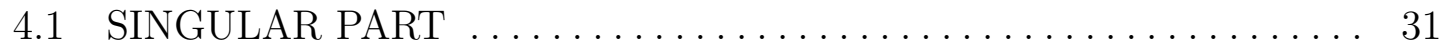

$4.2 \quad$ ADDING NON-SINGULAR PART OF THE KERNEL . . . . . . . . . . . 33

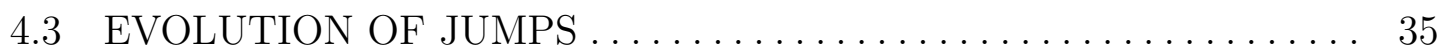

5. STRUCTURE OF PHOTON GENERALIZED DISTRIBUTION AMPLI-

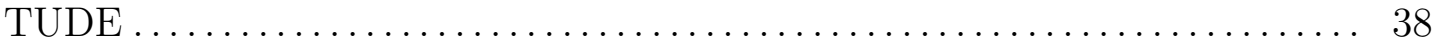

6. EVOLUTION OF THE JUMP PART OF THE TWO-PHOTON GDA .... 42

7. EVOLUTION OF THE CUSP PART OF THE TWO-PHOTON GDA . . . 48

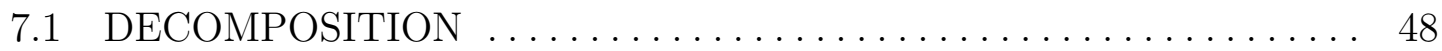

7.2 EVOLUTION OF THE LINEARIZED PART $\ldots \ldots \ldots \ldots \ldots \ldots \ldots \ldots \ldots . . \ldots . \ldots$

7.3 EVOLUTION OF CURVY PART $\ldots \ldots \ldots \ldots \ldots \ldots \ldots \ldots \ldots \ldots$

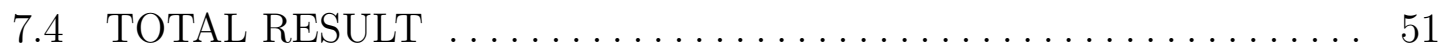

8. EVOLUTION IN DGLAP REGION $\ldots \ldots \ldots \ldots \ldots \ldots \ldots \ldots \ldots \ldots \ldots \ldots \ldots$ 8.1 EVOLUTION OF $(1-x)^{3}$ PARTON DISTRIBUTION FUNCTION • 55

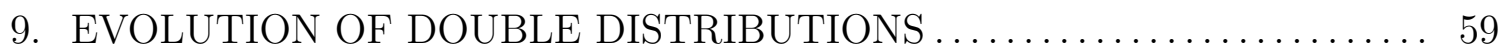

9.1 DOUBLE DISTRIBUTION BASICS $\ldots \ldots \ldots \ldots \ldots \ldots \ldots \ldots \ldots$

10. CONCLUSION AND FUTURE APPLICATIONS $\ldots \ldots \ldots \ldots \ldots \ldots \ldots \ldots \ldots 6$ 


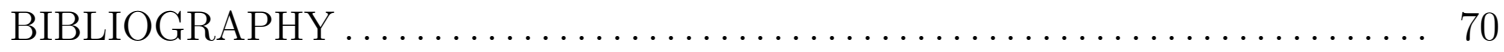

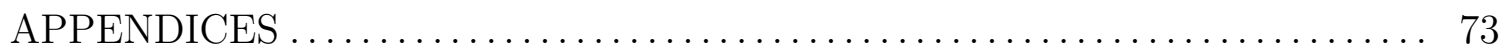

A. ANALYTIC EXPRESSIONS. . . . . . . . . . . . . . . . . . . . . . . 73

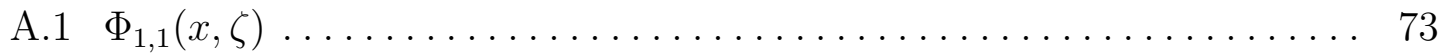

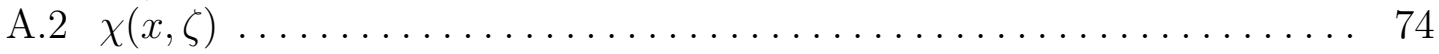

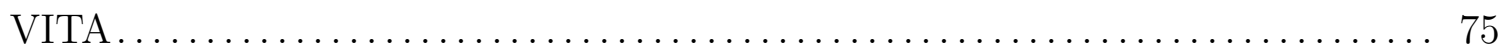




\section{LIST OF FIGURES}

Figure

1. Measurements of $\alpha_{s}$ as a function of $Q[24] \ldots \ldots \ldots \ldots \ldots \ldots \ldots \ldots$

2. Kinematic of DIS. The four-momenta of incoming and outgoing leptons are $k$ and $k^{\prime}, q=k^{\prime}-k, P$ is the four-momenta of the hadron with mass $M .7$

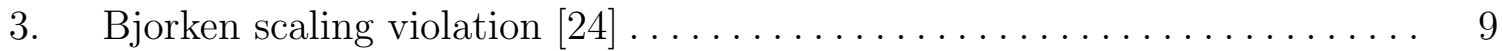

4. Illustration of how distribution amplitude is formed by infinite number of

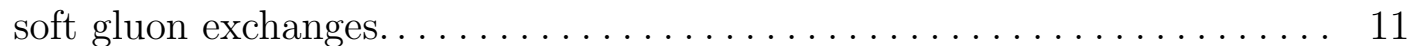

5. Triangle diagram contributing to the LO ERBL kernel . . . . . . . . . . . . . 13

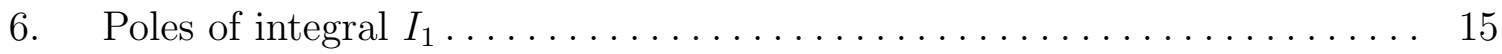

7. $\quad$ Self energy diagram contributing LO ERBL kernel . . . . . . . . . . . . . 17

8. Expansion of flat distribution amplitude in terms of Gegenbauer polyno-

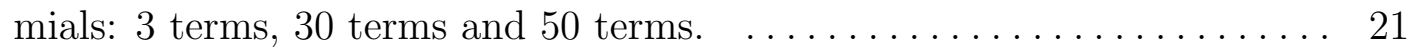

9. $\quad$ Flat function expanded in terms of Gegenbauer polynomials: 2 terms, 30

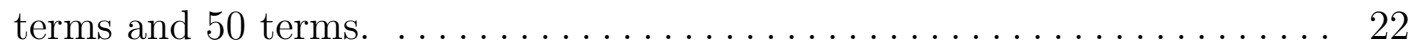

10. Expansion components $\Phi_{2}^{\text {sing }}(x)$ and $\Phi_{3}^{\text {sing }}(x) . \quad \ldots \ldots \ldots \ldots \ldots \ldots \ldots 24$

11. Normalization factor calculated for terms including only $\Phi_{0}(x)$ (shortdashed line), $\Phi_{0}(x)$ and $\Phi_{2}(x)$ (long-dashed line) and $\Phi_{0}(x), \Phi_{2}(x)$ and

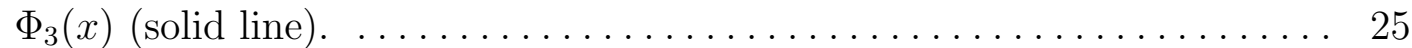

12. Evolution of the flat DA under the singular part of the evolution kernel: the curves shown correspond to $t=0$ (red), $t=0.3$ (black), $t=0.6$

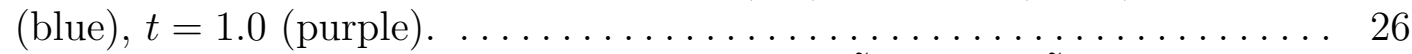

13. Expansion components in the full kernel case: $\tilde{\Phi}_{1}(x)$ and $\tilde{\Phi}_{2}(x) . \ldots \ldots$

14 . a) Normalization factor calculated for terms including only $\Phi_{0}(x)$ (dotdashed line), $\Phi_{0}(x)$ and $\Phi_{2}(x)$ (dashed line) and $\Phi_{0}(x), \Phi_{2}(x)$ and $\Phi_{3}(x)$

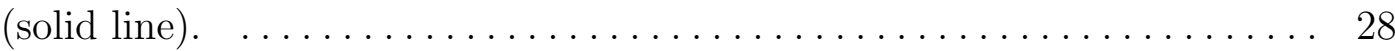

15. Evolution of flat DA for the full kernel case: the curves shown correspond to $t=0$ (red), $t=0.3$ (black), $t=0.6$ (blue), $t=1.0$ (purple). . . . . . 29

16. Flat DA at $t=0.2$; The dashed curve is the analytic calculation and the solid curve is the Gegenbauer expansion with 50 terms. . . . . . . . . . . . 29

17. Evolution of anti-symmetric DA at $t=0.2$ with the Gegenbauer expansion

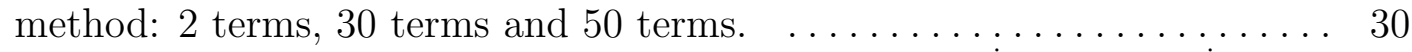

18. Expansion coefficients for the antisymmetric DA: a) $\Phi_{1}^{\text {sing }}(x)$, b) $\Phi_{2}^{\text {sing }}(x)$. $\quad 32$

19. Evolution of antisymmetric DA under the singular part of the kernel for

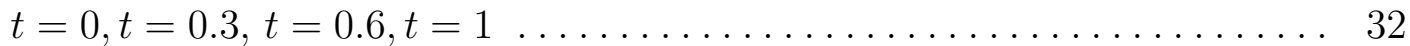

20. Expansion components $\Phi_{1}(x)$ (a) and $\Phi_{2}(x)(\mathrm{b})$ in the case of the full

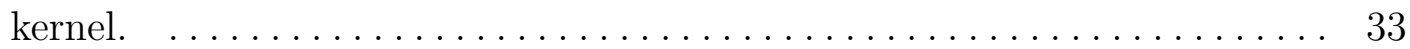

21. Evolution of the antisymmetric DA in the full kernel case. The curves for $t=0$ (red), $t=0.3$ (black), $t=0.6$ (blue), $t=1.0$ (purple) are shown. . . 34

22. Anti-symmetric DA at $t=0.2$; The dashed curve is the analytical calculation and the solid curve is the Gegenbauer expansion with 50 terms. ... 34

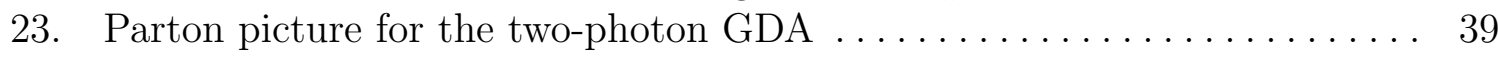

24. Two-photon GDA profile function $\Phi(x, \zeta)$ at values $\zeta=0.1 ; 0.2 ; 0.4$. . . . 40 
25. (a) The $x$-profile of GDA $\Phi_{1,0}(x, \zeta)$ at $\zeta=0.3$. (b) Initial function

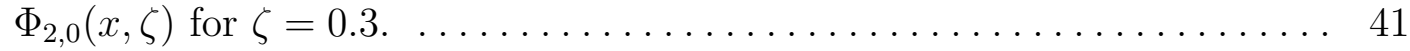

26. $\quad$ First iteration $\Phi_{1,1}(x, \zeta)$ for $\zeta=0.3 . \ldots \ldots \ldots \ldots \ldots \ldots \ldots \ldots \ldots \ldots \ldots$

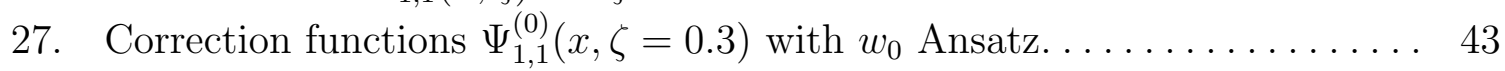

28. Correction functions $\Psi_{1,1}^{(0)}(x, \zeta=0.3)$ with modified Ansatz. . . . . . . . . . 44

29. Ansatz function $\Phi_{1}(x, \zeta=0.3, t)$ for $t=0,0.2,0.3,0.5 . \ldots \ldots \ldots \ldots$

30. Effect of inclusion of $\Psi_{1,1}(x, \zeta)$ correction for $t=0.2$ (a) and $t=0.4$ (b); $\zeta=0.3$ in both cases. Red line corresponds to $\varphi_{1}(x, \zeta, t)$ without correction and blue corresponds to $\varphi_{1}(x, \zeta, t)$ with correction. $\ldots \ldots \ldots .45$

31. (a) $\delta \Psi_{1}(x, \zeta, t),(\mathrm{b}) \delta \Psi_{1}(x, \zeta, t)(\zeta=0.2$ and $t=0.2) . \ldots \ldots \ldots \ldots \ldots \ldots$

32. (a) $\Phi(x, \zeta, t),(\mathrm{b}) \Phi(x, \zeta, t)$ and $\delta \Psi_{1}(x, \zeta, t)+\delta \Psi_{2}(x, \zeta, t) . \quad(\zeta=0.2$ and

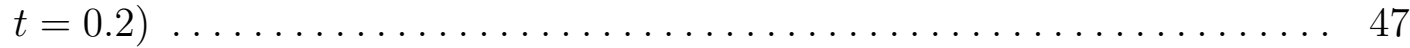

33. Initial function $\Phi_{2,0}(x, \zeta)$ for $\zeta=0.2 . \ldots \ldots \ldots \ldots \ldots \ldots \ldots \ldots \ldots \ldots \ldots$

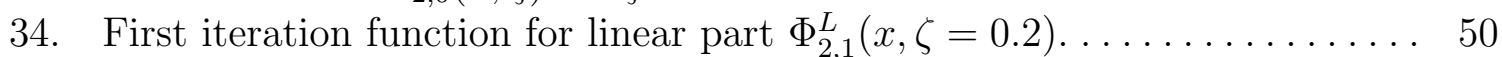

35. Left: Initilal curvy function $\Phi_{2.0}^{\mathrm{C}}(x, \zeta)$ for $\zeta=0.2$. Right: First iteration

function for curvy part $\Phi_{2,1}^{C}(x, \zeta)$ for $\zeta=0.2 . \ldots \ldots \ldots \ldots \ldots \ldots \ldots \ldots$

36. Evolution of $\phi_{2}(x, \zeta, t)$ at $t=0$ (red), $t=0.2$ (black), $t=0.3$ (blue) and

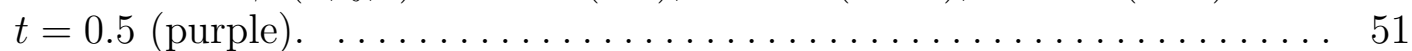

37. Evolution of total GDA $\Phi(x, \zeta, t)$ at $t=0$ (red), $t=0.1$ (black), $t=0.2$

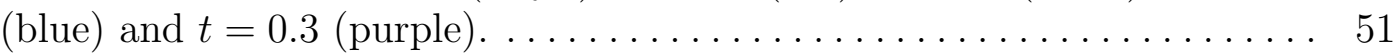

38. Comparison between analytic result presented here and the numerical result from Ref. [38] at $\zeta=0.4$ and $t=0$ (red), $t=0.1$ (black), $t=0.2$

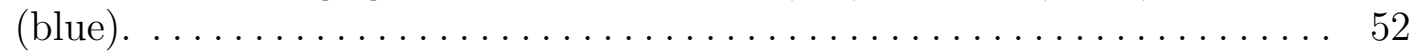

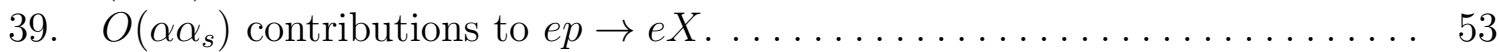

40. Expansion components $\rho_{1}^{\text {sing }}(x)$ and $\rho_{2}^{\text {sing }}(x) \ldots \ldots \ldots \ldots \ldots \ldots \ldots \ldots$

41. Blue line is the normalization including $\rho_{0}(x)$ and $\rho_{1}(x)$, green line is the normalization including $\rho_{0}(x), \rho_{1}(x)$ and $\rho_{2}(x)$ and red line is the normalization including $\rho_{0}(x), \rho_{1}(x), \rho_{2}(x)$ and $\rho_{3}(x)$ which was calculated

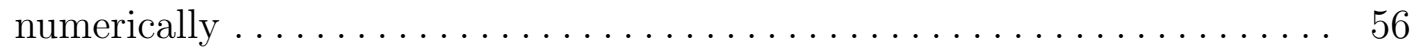

42. (a) Starting from blue line: $t=0$ (red), $t=0.2$ (black), $t=0.4$ (blue), $t=0.5$ (purple) and (b) Closer look to $0<x<0.2$ region $\ldots \ldots \ldots \ldots .57$

43. $\quad$ Starting from blue line: $t=0$ (red), $t=0.2$ (black), $t=0.4$ (blue), $t=0.5$ (purple) and (b) Closer look to $0<x<0.2$ region $\ldots \ldots \ldots \ldots \ldots \ldots 7$

44. Parton picture for double distributions $\ldots \ldots \ldots \ldots \ldots \ldots \ldots \ldots \ldots \ldots$

45. The triangle on the left is the integral domain before the change of variable $\left(z^{\prime} \rightarrow\right.$ eta $)$ and the right triangle is the integral line and the limits after the change of variables. The integral is divergent when $x=\xi$ and $y=\eta$ (point on $\zeta=1$ line) which is regularized by the "+" prescription. The line from 0 to $\zeta=1$ represents the line of integration for the second part

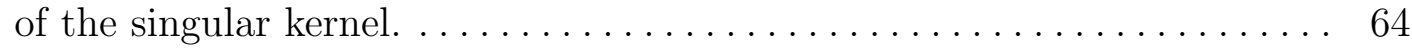

46. $\quad x$-profile at $t=0$ (red), $t=0.2$ (black) and $t=0.4$ (blue) and $y$ profiles at $x=0$ (red), $x=0.3$ (black) and $x=0.5$ (blue) for evolution of $(y(1-x-y))^{10} .66$ 


\section{CHAPTER 1}

\section{INTRODUCTION}

One of the most interesting problems in physics is understanding hadron structure. In 1973, Quantum Chromodynamics (QCD) was proposed as the theory of strong interactions [3] following the argument of Gell-Mann [4] and Zweig [5, 6] in 1964 which states that the hadrons are actually composed of more elementary constituents, called quarks and gluons, collectively known as partons. QCD was tested to high precision for high energy interactions, but, we still need more information about the structure of hadrons. The difficulties of getting information about hadron structure can be traced to a property of QCD named confinement. Due to confinement, observation of free quarks and gluons is not possible. But another property of QCD, asymptotic freedom, allows treatment of quarks and gluons as nearly free particles at short distances.

Asymptotic freedom, i.e. the smallness of the QCD coupling at high momenta, justifies the use of perturbative QCD (pQCD). At the same time, the increase of the QCD coupling in the low energy region $(\sim 1 \mathrm{GeV})$ prevents perturbative calculations for physical observables; therefore in this region parameters should be obtained from phenomenological analyses.

Reactions which allow access to the nonperturbative region attract the attention of many researchers among the hadronic physics community. Deep Inelastic Scattering (DIS) is one of the processes which has a very important role of determining the structure of hadrons. In DIS, the large virtuality of the probe enables factorization of perturbative (hard) and nonperturbative (soft) contributions. At short distances, the reaction is governed by pQCD, since the running coupling constant of QCD becomes small, providing weaker interaction between quarks and gluons. The rest of the information, which is non-pertubative, is factorized into DIS form factors and these form factors (structure functions) can be measured experimentally.

In QCD, the structure functions depend on the momentum $Q$ of the probe. The $Q^{2}$ dependence is known as the evolution of the distribution functions. In QCD, the scaling of structure functions is violated producing the evolution. This phenomena can be described in terms of scale dependent parton distribution functions, 
$f_{q / g}\left(x, \mu^{2}\right)$, where $\mu$ is usually the scale of the probe $Q$. The structure functions are the convolution of these parton distributions and coefficient functions, which are given as a power series in the strong coupling constant $\alpha_{s}(\mu)$. The evolution of the parton distributions is governed by the Dokshitzer-Gribov-Lipatov-Altarelli-Parisi (DGLAP) equations [7 9].

The photon-pion transition $\gamma \gamma^{*} \rightarrow \pi^{0}$ plays a key role among exclusive processes since it is the cleanest exclusive process to test QCD predictions. The scattering amplitude at large transferred momentum $Q^{2}$ factorizes as a convolution of the process independent distribution amplitude (DA), $\varphi(x, \mu)$, and process dependent hard scattering amplitude. The evolution of distribution amplitudes is governed by the Efremov-Radyushkin-Brodsky-Lepage (ERBL) equations [1, 2].

Another reaction which is receiving a lot of attention is Deeply Virtual Compton Scattering (DVCS). This reaction has lead to the proposal of new non-perturbative objects called Generelized Parton Distributions (GPDs) [10 15]. These functions are hybrids of form factors, parton distributions, and distribution amplitudes. Theoretical models for building GPDs have to satisfy some nontrivial requirements. One way to build GPDs satisfying these requirements is modeling with Double Distributions (DDs) [13, 14, 16. In particular, one may assume some simple Ansatz for a DD at a low scale $\mu$, and then evolve it into the moderately large momentum transfer region. To do this, one needs to incorporate efficient methods of QCD evolution.

In this thesis, we illustrate a new analytic method for evolution of distribution amplitudes and generalized distribution amplitudes. We also show how to extend our method for the DGLAP evolution equation and the evolution of double distributions.

Our approach to DA evolution has advantages over the standard method of expansion in Gegenbauer polynomials [1, 2] and over a straightforward iteration of an initial distribution with evolution kernel. Expansion in Gegenbauer polynomials requires an infinite number of terms in order to accurately reproduce functions in the vicinity of singular points. Straightforward iteration of an initial distribution produces logarithmically divergent terms at each iteration. In our method the logarithmic singularities are summed from the start, which immediately produces a continuous curve. Afterwards, in order to get precise results, only one or two iterations are needed. 
One of the motivations for studying the evolution of singular distribution amplitudes is modeling GPDs. A well known property of GPDs is that they are nonanalytic at border points $x= \pm \xi$. For one-loop diagrams, this non-analyticity may take the form of cusps, jumps, and even delta-functions. Thus, one needs to develop methods of evolution for singular initial distributions. A simple example of a singular distribution is given by flat distribution amplitudes, $\varphi(x)=$ const, which do not vanish at the $x=0, x=1$, boundaries of the DA support region. We first demonstrate the application of the method on flat DA. Our method allows to easily establish the major evolution pattern $(x \bar{x})^{t}$ with $t=\frac{2 C_{F}}{\beta_{0}} \ln [\ln (\mu / \Lambda)]$ for a flat DA, and provides an algorithm for an analytic calculation of corrections to it. The method was also applied to a DA $\varphi(x)=\operatorname{sign}(x-1 / 2)$ that has a jump at $x=1 / 2$, in the middle of the support interval, with DA being antisymmetric with respect to that point.

This method is then extended for studying the evolution of generalized distribution amplitudes (GDAs) [17]. Similarly to GPDs, these functions are non-analytic at kinematics-dependent points $x=\zeta, 1-\zeta$ inside the support interval. The evolution of GPDs is further complicated by the fact that GPD evolution kernels also depend on skewness $\zeta$ (or $\xi$ ). Unlike GPDs, GDAs evolve according to the same $\zeta$-independent ERBL kernels as the usual DAs, which allows us to concentrate on studying implications due to the non-analytic structure of the initial distribution. A particular object that we consider is the two-photon GDA [18] related to the reaction $\gamma^{*}(q) \gamma\left(q^{\prime}\right) \rightarrow \gamma\left(p_{1}\right) \gamma\left(p_{2}\right)$. In QCD lowest order, it is proportional to the $V V \rightarrow V V$ ERBL evolution kernel, but the evolution of its $\ln Q^{2}$ derivative, in the leading logarithm approximation, is governed by the $q q \rightarrow q q$ ERBL kernel.

The thesis is organized as follows: The rest of this chapter is dedicated to basic information necessary to explain some properties of our method including QCD, Deep Inelastic Scattering, and Factorization. In Chapter 2, we discuss the basic ideas of our method. In particular, we convert the evolution equation to the form in which the convolution integral has the structure of the "plus prescription" with respect to the integration variable $y$. The evolution equation is further simplified by choosing the Ansatz absorbing the extra term generating contributions, that are logarithmically singular at the end points of the support region. Applying this method for an initially flat DA in Chapter 3, we find that, for any small positive value of the evolution parameter $t$, the flat DA evolves into a function vanishing at the end points with its shape dominated by the $[x(1-x)]^{t}$ factor. Then we analytically calculate the lowest 
corrections to this approximation. In Chapter 4, we also apply this method to the antisymmetric flat DA that initially takes opposite values for $x<1 / 2$ and $x>1 / 2$. Such a DA is the simplest example of an initial distribution with a jump inside the support region (in this case in the middle of the region). For further applications, we consider a case of an antisymmetric jump $\phi\left(x=\zeta_{-}\right)=-\phi\left(x=\zeta_{+}\right)$at an arbitrary position $x=\zeta$ inside the support region. Afterwards, we derive the formulas that are used in Chapters 5-7, where we apply the approach to the evolution of the twophoton generalized distribution amplitude $\psi^{q}\left(x, \zeta, Q^{2}\right)$. Its logarithmic derivative with respect to $Q^{2}$ satisfies the ERBL evolution equation, with initial conditions given by a function $\varphi(x, \zeta)$ that has both jumps (discontinuities in the value of the function $\varphi(x, \zeta))$ and cusps (discontinuities in the value of the derivative $\partial \varphi(x, \zeta) / \partial x$ ) at the "border" points $x=\zeta, x=1-\zeta$. The structure of $\varphi(x, \zeta)$ is discussed in Chapter 5 , where it is proposed to split it into a part that has antisymmetric jumps at the border points, and a continuous remainder that has cusps there. Evolution of the "jump" part of the two-photon GDA is considered in Chapter 6, while evolution of the "cusp" part is considered in Chapter 7. In Chapter 8, we demonstrate the application of our method to DGLAP evolution equation, with a nonsingular parton distribution function. In the last chapter, we demonstrate the application of a modified version of our method to evolution of double distributions.

\subsection{QUANTUM CHROMODYNAMICS}

The fundamental theory of strong interactions is Quantum Chromodynamics (QCD). QCD is a non-Abelian gauge theory with gauge group $\mathrm{SU}(3)$. The theory is based on renormalizability of gauge field theories discovered by 't Hooft and Veltman [19 21]. QCD involves local symmetries and the force carriers for QCD are colored gluons, which have zero mass and spin 1. QCD is based on the invariance under non-Abelian SU(3) group of local phase transformations among three colors, with the Lagrangian

$$
L_{Q C D}=-\frac{1}{4} F_{\mu \nu}^{(a)} F^{(a) \mu \nu}+i \sum_{q} \bar{\psi}_{q}^{i}\left[\gamma \mu\left(D_{\mu}\right)_{i j}+i m\right] \psi_{q}^{j},
$$

where $F_{\mu \nu}$ is the non-abelian field strength tensor

$$
F_{\mu \nu}^{(a)}=\partial_{\mu} A_{\nu}^{a}-\partial_{\nu} A_{\mu}^{a}-g_{s} f_{a b c} A_{\mu}^{b} A_{\nu}^{c}
$$


and $\left(D_{\mu}\right)_{i j}$ is the covariant derivative

$$
\left(D_{\mu}\right)_{i j}=\delta_{i j} \partial_{\mu}+i g_{s} \sum_{a} \frac{\lambda_{i j}^{a}}{2} A_{\mu}^{a} .
$$

$g_{s}$ is the QCD coupling constant and $f_{a b c}$ are the structure constants of SU(3) algebra. The term $g_{s} f_{a b c} A_{\mu}^{b} A_{\nu}^{c}$ is very essential. It corresponds to the interaction of gluon fields with each other through three or four gluon vertices. The only parameters of the theory are the coupling constant $g_{s}$ and the masses of quarks. For intermediate momenta $\sim 1-10 \mathrm{GeV}$, the masses of light quarks might be set to zero while the masses of heavy quarks might be set to be infinitely large. Thus, the only parameter in this situation is the coupling constant $g_{s}$.

The coupling "constant" between quarks is not really a constant. On the contrary, it depends on the distance between quarks. One may assume that a dimensionless QCD observable $R$ should be independent of $Q^{2}$ for $Q \gg m_{q}$. The only energy scales in the QCD Lagrangian are the quark masses. Since the relevant ones are very light, it would be expected that the scaling property would be set at low $Q^{2}$. However, this is not true in a renormalizable field theory. A scale is introduced when calculating the observable $R$ with perturbation theory because of logarithmically diverging integrals. In order to renormalize the theory, a scale should be introduced. As a result of this, the dimensionless observable $R$ does not scale anymore. It has logarithmic scaling violations, so the coupling constant $\alpha_{s}=g_{s}^{2} / 4 \pi$ of QCD becomes a running coupling constant. Its running is controlled by the beta function. The renormalization group equation may be solved exactly at the one loop level. One can then derive $\alpha_{s}$ at some scale $Q$, as a function of its value at the renormalization scale $\mu$,

$$
\alpha_{s}\left(Q^{2}\right)=\frac{\alpha_{s}\left(\mu^{2}\right)}{1+\left(\beta_{0} \alpha_{s}\left(\mu^{2}\right) / 4 \pi\right) \ln \left(Q^{2} / \mu^{2}\right)} \quad \text { with } \beta_{0}=\frac{11}{3} n_{c}-\frac{2}{3} n_{f},
$$

where $n_{c}$ is the number of quark colors and $n_{f}$ is number of "active" (those not treated as infinitely heavy) flavors in the theory. One can rewrite $\alpha_{s}\left(Q^{2}\right)$ as

$$
\alpha_{s}\left(Q^{2}\right)=\frac{4 \pi}{\beta_{0} \ln \left(Q^{2} / \Lambda^{2}\right)} .
$$

The parameter $\Lambda$ is the QCD scale. Its numerical value for three active quark flavor is about $200 \mathrm{MeV}$. From this relation, it can be seen that $\alpha_{s} \rightarrow 0$ as $Q \rightarrow \infty$ (asymptotic freedom) [22, 23] and, on the other hand, QCD becomes strongly coupled at $Q \sim \Lambda$. 


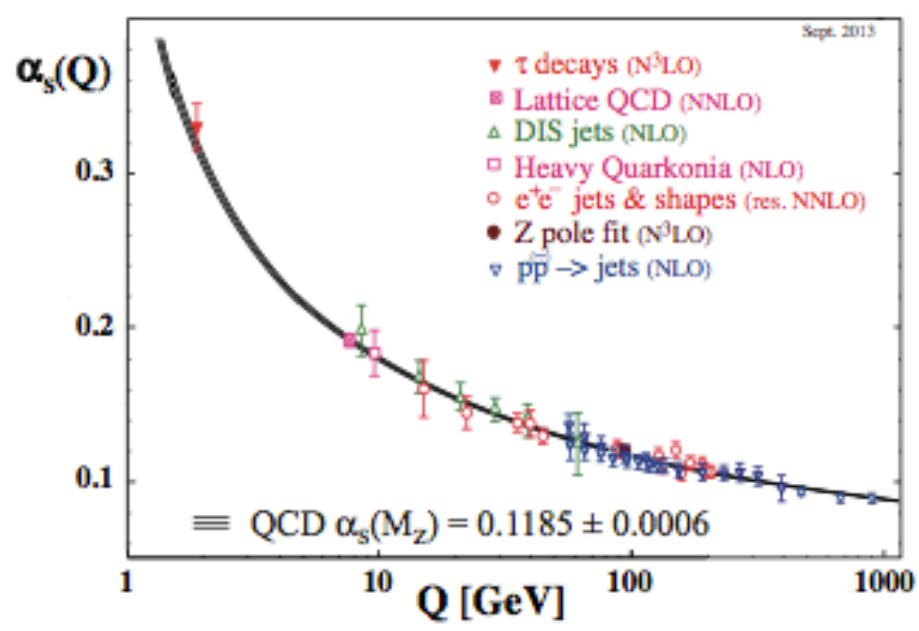

FIG. 1: Measurements of $\alpha_{s}$ as a function of $Q$ [24].

In other words, the momentum scale $\Lambda$ is the scale at which $\alpha_{s}$ becomes strong as $Q^{2}$ decreases. In Fig. 1, a summary of measurements of $\alpha_{s}\left(Q^{2}\right)$ is presented as a function of energy scale $Q$. The data points are based on the QCD calculations for combined world average value of $\alpha_{s}\left(M_{Z}\right)$. 


\subsection{DEEP INELASTIC SCATTERING}

One of the ways to test perturbative QCD is deep inelastic scattering (DIS). "Deep" means that wavelength of the photon is much smaller than the size of the target hadron. Thus, the photon can probe very small distances compared to the hadron. "Inelastic" corresponds to high energy leptons destructing the target hadron and causing the formation of new hadrons; i.e. the target hadron is converted into a large number of hadrons in the process.

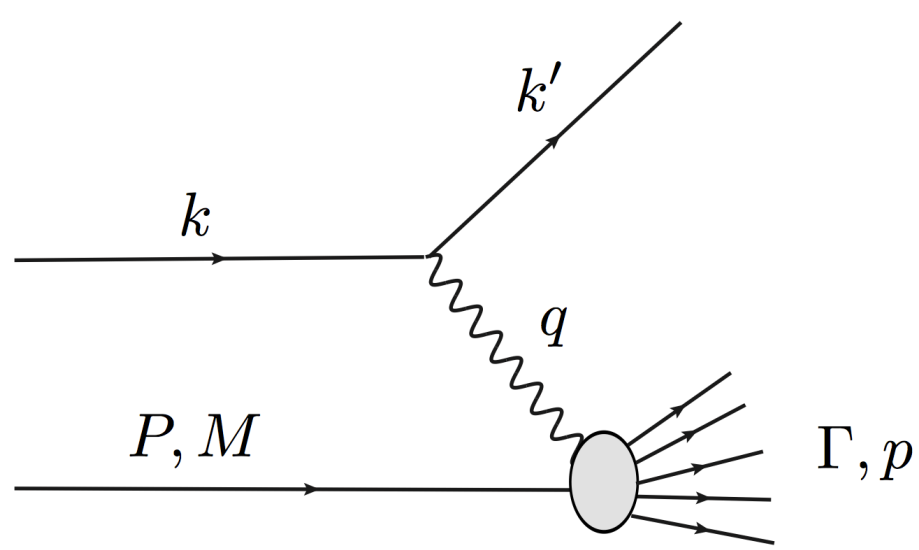

FIG. 2: Kinematic of DIS. The four-momenta of incoming and outgoing leptons are $k$ and $k^{\prime}, q=k^{\prime}-k, P$ is the four-momenta of the hadron with mass $M$.

The general form of the scattering is $l+h \rightarrow l^{\prime}+X$, where $l$ and $l^{\prime}$ are leptons, $h$ is the hadron target and $X$ is the sum of all possible hadron states. The incoming momenta of the hadron and lepton are $P$ and $k$, respectively. Outgoing momentum of the lepton is $k^{\prime}$ and the transferred momentum $q$ is $q=k-k^{\prime}$. The DIS process is characterized by the following invariant quantities:

$$
\begin{aligned}
y & =\frac{q \cdot P}{k \cdot P}=\frac{\nu}{E}(\text { the fraction of lepton's energy }) \\
W^{2} & \left.=(P+q)^{2}=M^{2}+2 M \nu-Q^{2} \text { (mass squared of the final system }\right), \\
s & =(k+P)^{2}=\frac{Q^{2}}{x y}+M^{2}(\mathrm{CM} \text { energy squared })
\end{aligned}
$$


The virtual photon sub-process is characterized by the following quantities

$$
\begin{aligned}
Q^{2} & =-q^{2}=2\left(E E^{\prime}-\vec{k} \cdot \overrightarrow{k^{\prime}}\right) \\
& \approx 4 E E^{\prime} \sin ^{2}(\theta / 2) \text { (neglecting the lepton masses), } \\
\nu & =\frac{q \cdot P}{M}=E-E^{\prime} \text { (the lepton's energy loss in the hadron rest frame), } \\
x_{B j} & =\frac{Q^{2}}{2 M \nu} \text { (the fraction of the hadron's momentum carried by struck quark), }
\end{aligned}
$$

which are called Bjorken variables. The deep inelastic limit (Bjorken limit) is specified by

$$
Q^{2}, M \nu \gg \Lambda^{2}, \quad x \rightarrow \text { fixed }
$$

If the incoming and outgoing leptons are electrons or muons, the hadron is probed by electromagnetic interactions. In pure electromagnetic case, the virtual vector particle is a photon. Neglecting the contribution of weak currents, the relevant Lagrangian is given by the following expression,

$$
L_{\text {int }, e m}=e J_{e m}^{\mu} A_{\mu}
$$

where

$$
J_{e m}^{\mu}=\sum_{q} Q_{q} \bar{q} \gamma^{\mu} q
$$

is the corresponding electromagnetic (EM) current, with $Q_{q}$ being the charge of the quark $q$.

The scaling phenomenon was proposed in 1969 by Bjorken [25] before the parton model. Scaling means that as $Q^{2} \rightarrow \infty$ the structure functions become independent of $Q^{2}$,

$$
\left.F_{i}\left(x, Q^{2}\right)\right|_{Q^{2} \rightarrow \infty} \Rightarrow F(x)
$$

This relation can be explained by assuming that the transverse momentum of partons in the infinite-momentum frame of the proton is small. In other words, if quarks were non-interacting, then no further structure would be resolved no matter how much $Q^{2}$ increases. According to QCD predictions, however, with increasing $Q^{2}$ quarks start to emit hard gluons. This emission causes logarithmic scaling violation. While the probability of finding a quark at small $x$ increases with $Q^{2}$, the probability of 
finding it at high $x$ decreases, since high momentum quarks emit gluons while losing momentum. Emission of hard gluons from quarks leads to the evolution of structure functions. As $Q^{2} \rightarrow \infty$, the number of gluons emitted by quarks increases at high $x$. These emitted gluons may split into $q \bar{q}$ pairs or two gluons. This process causes both the softening of the initial quark momentum distributions and to the growth of gluon density and $q \bar{q}$ sea as $x$ decreases.

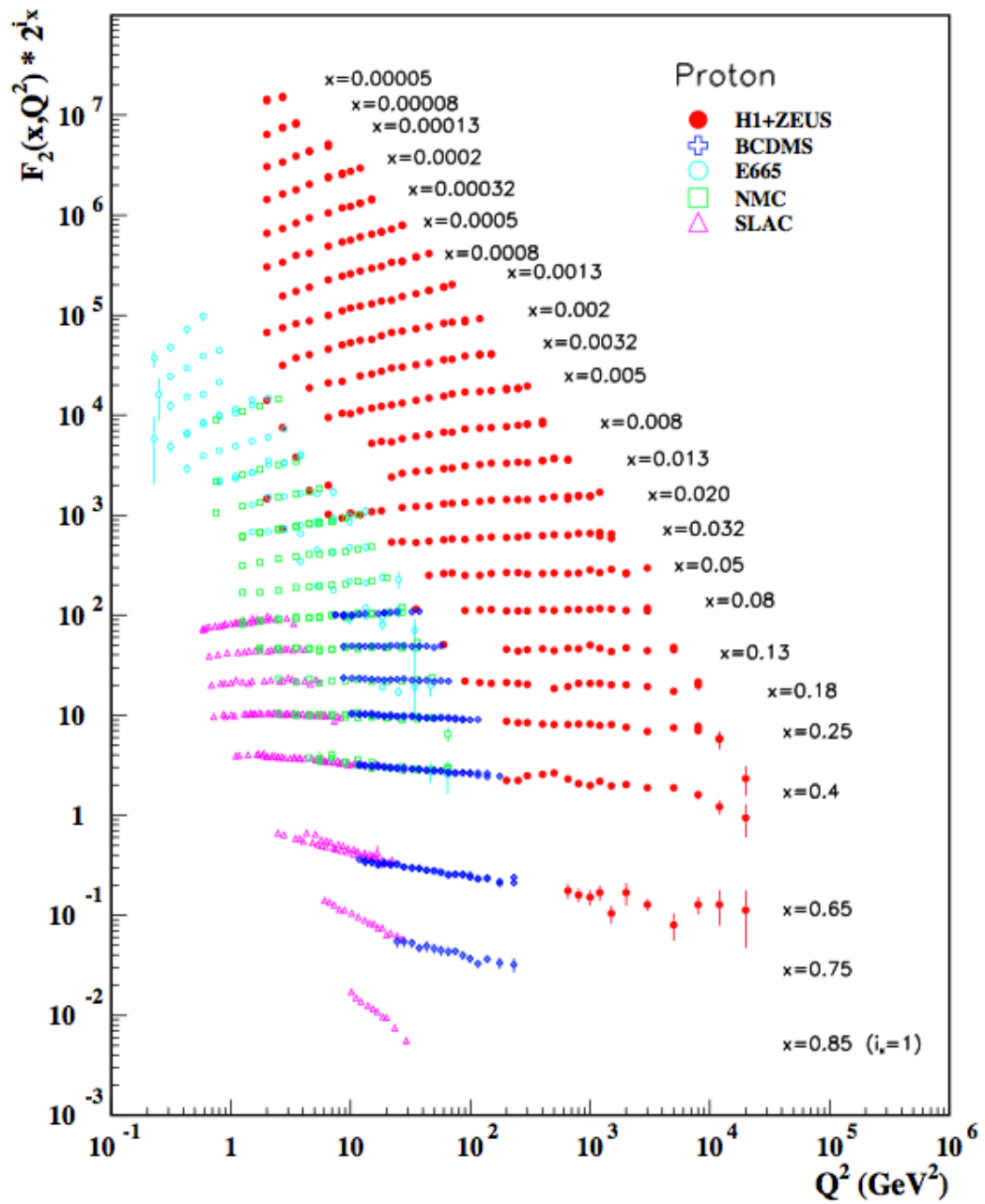

FIG. 3: Bjorken scaling violation [24] 


\subsection{FACTORIZATION}

The scope of perturbative QCD (pQCD) would be rather limited if its predictions were only limited to infrared safe observables. Thus, probing the partonic structure of hadrons or making predictions of high energy cross section processes with the existing information of hadron structure would be impossible. Factorization [26, 27] is one of the important properties of $\mathrm{pQCD}$ which provides the foundation of the parton model. Partonic cross sections which are not infrared safe by themselves are factorizable into short-distance (hard) and long-distance (soft) parts. The shortdistance part is infrared safe while the long-distance part has infrared singularities. This separation (factorization) provides the connection of partonic cross sections, which are calculable, to hadronic cross sections which are experimentally measurable.

Factorization pertains to the infrared singularities associated with long-distance interactions. These singularities are absorbed into non-perturbative but experimentally measurable objects. These objects are parton distribution functions (PDF) in the inclusive processes such as DIS and DA in exclusive processes such as meson production in photon-photon collisions or DVCS. In some sense, factorization is similar to the absorption of ultraviolet divergences into measurable physical constants in renormalization theory.

The factorization may be illustrated by the example of the pion form factor $F_{\pi}\left(Q^{2}\right)$. For large $Q^{2}$, it factorizes as

$$
F_{\pi}\left(Q^{2}\right)=\int_{0}^{1} d x \int_{0}^{1} d y \varphi^{\dagger}\left(y, Q^{2}\right) T\left(x, y, Q^{2}\right) \varphi\left(x, Q^{2}\right) .
$$

A perturbation expansion of amplitude $T\left(x, y, Q^{2}\right)$ is given by

$$
T\left(x, y, Q^{2}\right)=\alpha_{s}\left(Q^{2}\right) T_{B}\left(x, y, Q^{2}\right)+\alpha_{s}^{2}\left(Q^{2}\right) T_{2}\left(x, y, Q^{2}\right)+\ldots
$$

$T_{B}$ is the Born term of the hard scattering amplitude and is equal to

$$
T_{B}\left(x, y, Q^{2}\right)=\frac{4}{3} \frac{16 \pi}{Q^{2}(1-x)(1-y)},
$$

and $\varphi\left(x, Q^{2}\right)$ is the pion distribution amplitude. It is related to the pion wave function and describes how the longitudinal momentum of a fast-moving pion is shared among constituents belonging to a particular Fock component. All the soft gluon contributions, i.e. infrared singularities of the form $\left[\alpha_{s} \log \left(Q^{2} / m^{2}\right)\right]^{n}$ are absorbed into $\varphi\left(x, Q^{2}\right)$, where $m$ is the mass used to regularize the divergence. 
Fig. 4 depicts how the distribution amplitude $\varphi\left(x, Q^{2}\right)$ is generated from infinite number of soft gluon exchanges.

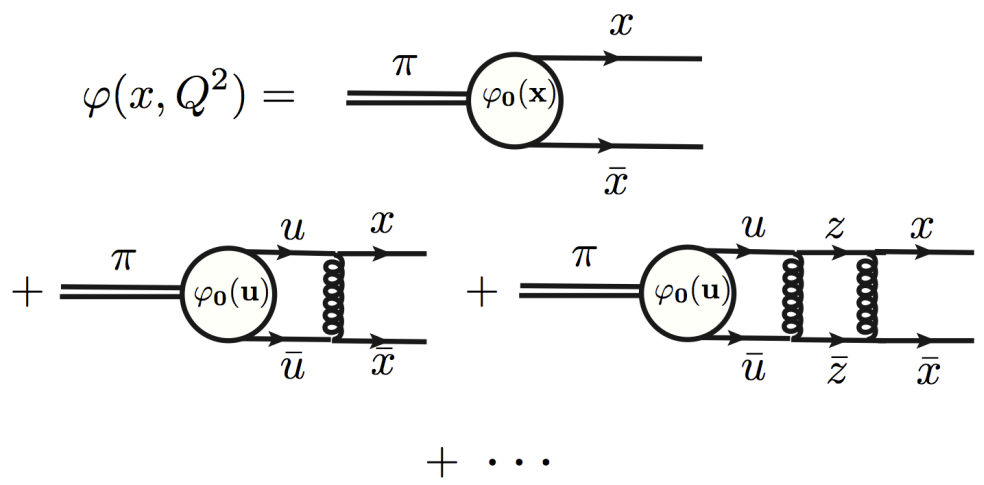

FIG. 4: Illustration of how distribution amplitude is formed by infinite number of soft gluon exchanges.

In this chapter, we explained elementary information necessary for understanding the basics of the analytic method for the evolution of singular distribution amplitude. Next chapter we demonstrate the basics of the ERBL evolution and the evolution kernel, We also summarize the standard way of calculating the ERBL evolution equation. 


\title{
CHAPTER 2
}

\section{EVOLUTION OF SINGULAR DISTRIBUTION}

\author{
AMPLITUDES
}

\subsection{EVOLUTION EQUATION: BASICS}

Evolution equations predict the parton distributions for any scale $\mu^{\prime}$ using the measured parton distribution at a scale $\mu$. In order to describe this dependence of the parton distributions on the renormalization scale, $\mu$ and $\mu^{\prime}$ should be large enough so that running coupling constants $\alpha_{s}(\mu)$ and $\alpha_{s}\left(\mu^{\prime}\right)$ are small. Evolution kernels are the key objects of evolution equations. In the following section, derivation of the leading-order (LO) evolution kernel for the DA in hard exclusive reactions (ERBL kernel) is illustrated.

\subsubsection{DERIVATION OF LO ERBL KERNEL}

The leading order (LO) ERBL kernel represents the amplitude for a quark with fractional momentum $y$ and antiquark with $(1-y)$ to became a quark with fractional momentum $x$ and antiquark with $(1-x)$ by exchange of a gluon. There are two diagrams contributing to the LO kernel, the triangle diagram and the self energy diagram. We will begin with calculation of triangle diagram.

The triangle diagram in Fig. $\quad 5$ a contributes to the matrix element $\left\langle 0 \mid \bar{\psi}(-z / 2) \gamma^{\nu} \psi(z / 2) P\right\rangle$. We can expand this expression in a Taylor series

$$
\left\langle 0\left|\bar{\psi}(-z / 2) \gamma^{\nu} \psi(z / 2)\right| P\right\rangle=\sum_{N=0}^{\infty} \frac{z_{\mu_{1}} \ldots z_{\mu_{N}}}{N !}\left\langle 0\left|\bar{\psi}(0) \gamma^{\nu} \frac{\stackrel{\leftrightarrow}{\partial}_{\mu_{1}}}{2} \frac{\stackrel{\leftrightarrow}{\partial}_{\mu_{2}}}{2} \ldots \frac{\stackrel{\leftrightarrow}{\partial}_{\mu_{N}}}{2} \psi(0)\right| P\right\rangle
$$

After the Taylor expansion, non-local operators become local operators (Fig. 5b). Matrix elements of twist-2 local operators here may be parametrized by

$$
i^{N+1}\left\langle 0\left|\psi(0)\left\{\gamma^{\nu} \frac{\stackrel{\leftrightarrow}{\partial}_{\mu_{1}}}{2} \frac{\stackrel{\leftrightarrow}{\partial}_{\mu_{2}}}{2} \ldots \frac{\stackrel{\leftrightarrow}{\partial}_{\mu_{N}}}{2}\right\} \psi(0)\right| P\right\rangle=\left\{P^{\nu} P^{\mu_{1}} P^{\mu_{2}} \ldots P^{\mu_{N}}\right\} f_{N}
$$




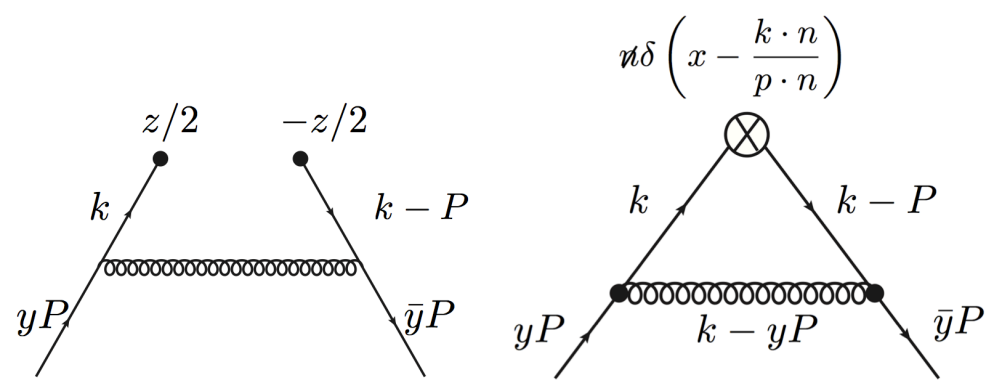

a

b

FIG. 5: Triangle diagram contributing to the LO ERBL kernel

where $\{\ldots\}$ corresponds to the symmetric traceless combination of the momentum vectors. One way to get symmetric traceless combinations is to multiply the tensor by $n_{\nu} n_{\mu_{1}} n_{\mu_{2}} \ldots n_{\mu_{N}}$ with $n$ having the property $n^{2}=0$.

The loop momentum integral for the reduced matrix elements $f_{N}$ can be written then as

$$
f_{N}=\int d^{4} k \not h(k n)^{N} /(P n)^{N+1} \ldots .
$$

The evolution of the DA may be written in matrix form as introduced in Ref. [1]

$$
\mu \frac{d}{d \mu} f_{N}(\mu)=\sum_{k=0}^{N} Z_{N k} f_{k}(\mu) .
$$

Another way to write the evolution equation of $\varphi(x)$ is the kernel form [2]. To this end, treating $f_{N}$ 's as the moments of some function $\varphi(x)$

$$
f_{N}=\int_{0}^{1} x^{N} \varphi(x) d x,
$$

we introduce the parton distribution amplitude $\varphi(x)$. The integral over $k$ for $\varphi(x)$ may be written as

$$
\varphi(x)=\int d^{4-2 \epsilon} k \not h \delta\left(x-\frac{k n}{P n}\right) \ldots
$$

Defining the kernel

$$
\int_{0}^{1} V(x, y) x^{N} d x=\sum_{k=0}^{N} Z_{N k} y^{N},
$$


we convert the matrix form of evolution equation into the kernel form as

$$
\mu \frac{d}{d \mu} \varphi(x, \mu)=\int_{0}^{1} V(x, y) \varphi(x, \mu) d y .
$$

In our example of the calculation of the non-forward ERBL kernel $V(x, y)$, according to 18 , the vertex can be replaced by $\not h \delta\left(x-\frac{k n}{P n}\right)$. The one loop diagram depicted in Fig. 5 in light-cone gauge is governed by

$$
\begin{aligned}
V_{1}(x, y) & =\int d^{4-2 \epsilon} k \frac{\operatorname{Tr}\left\{\not P \gamma^{\mu}(\not k-\not P) \not h k \gamma^{\nu}\right\}}{\left(k^{2}+i \epsilon\right)\left((k-P)^{2}+i \epsilon\right)\left((k-y P)^{2}+i \epsilon\right)} \\
& \times\left[g_{\mu \nu}-\frac{(k-y P)_{\mu} n_{\nu}+(k-y P)_{\nu} n_{\mu}}{(k-y P) \cdot n+i \epsilon}\right] \delta\left(x-\frac{k n}{P n}\right) .
\end{aligned}
$$

To handle the UV divergences, we here used dimensional regularization [28]. The momentum $k$ in light-cone components may be written using Sudakov parametrization [29] as

$$
\begin{aligned}
k & =x P+\alpha n+k_{\perp}, \\
d^{4-2 \epsilon} k & =d^{2-2 \epsilon} k_{\perp} d \alpha d x \\
k^{2} & =k_{\perp}^{2}+\alpha x \\
P^{2} & =n \cdot k_{\perp}=n \cdot p=0,2 P \cdot n=1 .
\end{aligned}
$$

The relation of $\gamma$ matrices in $4-2 \epsilon$ dimension $\gamma^{\mu} \gamma^{\nu} \gamma^{\rho} \gamma^{\sigma} \gamma_{\mu}=-2 \gamma^{\sigma} \gamma^{\rho} \gamma^{\nu}+2 \epsilon \gamma^{\nu} \gamma^{\rho} \gamma^{\sigma}$ provides simplification of trace,

$$
\gamma^{\mu}(\not k-\not P) h \not k \gamma^{\nu}=-2 \not k h(\not k-\not P)+2 \epsilon(\not k-\not P) h \not k .
$$

Analysis of integral $I$ can be divided in two parts: the part woth metric tensor $g_{\mu \nu}$ and the rest of the terms. The integral with metric tensor, $I_{1}$, is given by

$$
\begin{aligned}
I_{1} & =\int d^{4-2 \epsilon} k \frac{\operatorname{Tr}\{\not P \not k n k k\}}{\left(k^{2}+i \epsilon\right)\left((k-P)^{2}+i \epsilon\right)\left((k-y P)^{2}+i \epsilon\right)} \delta\left(x-\frac{k n}{P n}\right) \\
& =4(-2(2-2 \epsilon)) \int d^{4-2 \epsilon} k \frac{2(P \cdot k)(k \cdot n)-(P \cdot n) k^{2}}{\left(k^{2}+i \epsilon\right)\left((k-P)^{2}+i \epsilon\right)\left((k-y P)^{2}+i \epsilon\right)} \\
& \times \delta\left(x-\frac{k n}{P n}\right) \\
& =4((2-2 \epsilon)) \int d^{2-2 \epsilon} k_{\perp} d \alpha \\
& \times \frac{k_{\perp}^{2}}{\left(\alpha x+k_{\perp}^{2}+i \epsilon\right)\left(\alpha(x-1)+k_{\perp}+i \epsilon\right)\left(\alpha(x-y)+k_{\perp}^{2}+i \epsilon\right)} .
\end{aligned}
$$


Integral $I_{1}$ has three poles in the intervals $x>1, x<0,0<x<y$ and $y<x<1$. Fig. 6 shows the calculation of poles in these regions. In the intervals $x>1$ and $x<0$, the contour could be closed from top and from bottom, respectively producing zero result. For the intervals $0<x<y$ and $y<x<1$, the contour could be closed in such a way that only one pole is included inside the contour. Results for the intervals

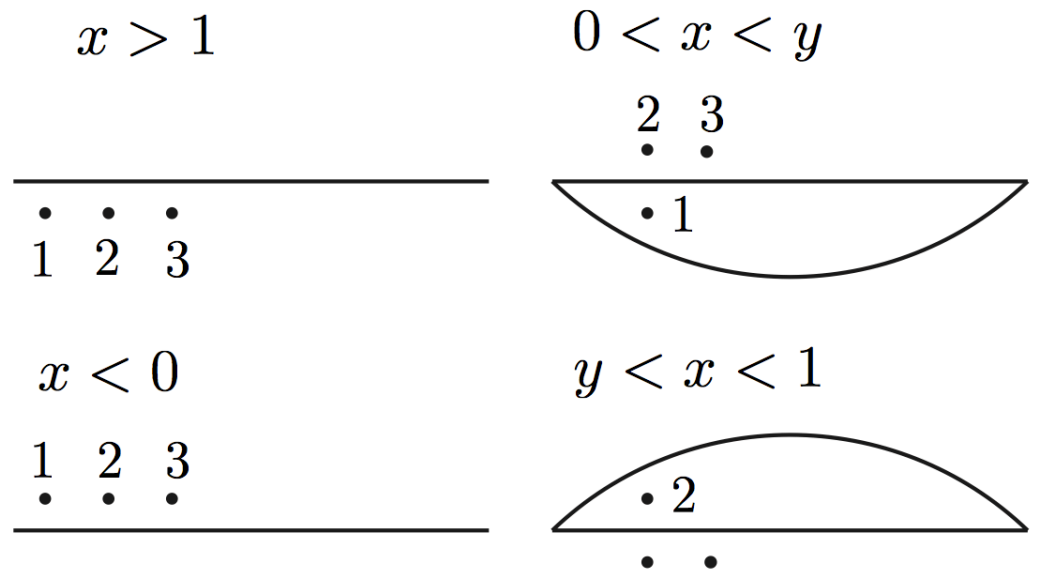

FIG. 6: Poles of integral $I_{1}$

$0<x<y$ and $y<x<1$ are

$$
\begin{aligned}
& \text { when } 0<x<y \Rightarrow 4(2-2 \epsilon) \int \frac{d^{2-2 \epsilon} k_{\perp}}{k_{\perp}^{2}} \frac{x}{y} \\
& \text { when } y<x<1 \Rightarrow-4(2-2 \epsilon) \int \frac{d^{2-2 \epsilon} k_{\perp}}{k_{\perp}^{2}} \frac{\bar{x}}{\bar{y}} .
\end{aligned}
$$

Integral $I_{1}$ becomes

$$
I_{1}=4(2-2 \epsilon) \int \frac{d^{2-2 \epsilon} k_{\perp}}{k_{\perp}^{2}}\left[\frac{x}{y} \theta(x<y)-\frac{\bar{x}}{\bar{y}} \theta(\bar{x}<\bar{y})\right] .
$$

The second part of the integration, $I_{2}$ is

$$
\begin{aligned}
I_{2}= & \int d^{4-2 \epsilon} k \\
& \frac{\operatorname{Tr}\{\not P(\not k-y \not P)(\not k-\not P) h \not k h\}+\operatorname{Tr}\{\not P \not h(\not k-\not P) \not h k(\not k-y \not P)\}}{\left(k^{2}+i \epsilon\right)\left((k-P)^{2}+i \epsilon\right)\left((k-y P)^{2}+i \epsilon\right)(((k-y P) \cdot n)+i \epsilon)} .
\end{aligned}
$$

where traces can be simplified as

$$
\begin{aligned}
& \operatorname{Tr}\{\not P(k-y \not P)(\not k-\not P) \not \not k \not h\}=8(k \cdot n)(P \cdot n)\left[k^{2}-2 k \cdot P\right] \\
& \operatorname{Tr}\{\not P \not h(\not k-\not P) \not k k(\not k-y \not P)\}=8 k^{2}(P \cdot n)[k \cdot n-P \cdot n],
\end{aligned}
$$


The integration becomes

$$
\begin{aligned}
& I_{2}=\int d^{2-2 \epsilon} k_{\perp} d \alpha d \beta \\
& \quad \frac{4 \alpha \beta(\beta-1)+k_{\perp}^{2}(4 \beta-2) \delta(x-\beta)}{\left(\alpha \beta+k_{\perp}^{2}+i \epsilon\right)\left(\alpha(\beta-1)+k_{\perp}+i \epsilon\right)\left(\alpha(\beta-y)+k_{\perp}^{2}+i \epsilon\right)(\beta-y) / 2} .
\end{aligned}
$$

In the interval $0<x<y$, integration $I_{2}$ is

$$
\begin{aligned}
I_{2} & =4(2-2 \epsilon) \int d^{2-2 \epsilon} k_{\perp} \frac{-4(x-1) k_{\perp}^{2}+k_{\perp}^{2}(4 x-2)}{x\left(-\frac{k_{\perp}^{2}}{x}(x-1)+k_{\perp}^{2}\right)\left(-\frac{k_{\perp}^{2}}{x}(x-y)+k_{\perp}^{2}\right)(x-y)} \\
& =\int \frac{d^{2-2 \epsilon} k_{\perp}}{k_{\perp}^{2}} \frac{x}{y(x-y)} .
\end{aligned}
$$

In the interval $y<x<1, I_{2}$ is

$$
\begin{aligned}
4(2-2 \epsilon & \int d^{2-2 \epsilon} k_{\perp} \frac{-4 x k_{\perp}^{2}+k_{\perp}^{2}(4 x-2)}{(x-1)\left(-\frac{k_{\perp}^{2}}{x-1} x+k_{\perp}^{2}\right)\left(-\frac{k_{\perp}^{2}}{x-1}(x-y)+k_{\perp}^{2}\right)(x-y)} \\
& =-\int \frac{d^{2-2 \epsilon} k_{\perp}}{k_{\perp}^{2}} \frac{\bar{x}}{\bar{y}(x-y)} .
\end{aligned}
$$

By adding all the components of integral $I$, one gets the following expression,

$$
I=4(2-2 \epsilon) \int \frac{d^{2-2 \epsilon} k_{\perp}}{k_{\perp}^{2}}\left[\left(\frac{x}{y}+\frac{x}{y(x-y)}\right) \theta(x<y)-\left(\frac{\bar{x}}{\bar{y}}+\frac{\bar{x}}{\bar{y}(x-y)}\right) \theta(y<x)\right]
$$

It is important to notice that integral $I$ is divergent when $x=y$.

The self energy diagrams are the other contributing diagrams to the LO ERBL kernel. We are not representing all the steps for the self energy diagram since all the necessary procedure is explained during the calculation of the one loop integral. The self energy contribution, $V_{2}(x, y)$ is equal to

$$
V_{2}(x, y)=-\delta(y-x) \int_{0}^{1} d z V_{1}(z, y)
$$

This term provides canceling $x=y$ divergencies in $V_{1}(x, y)$. Combination

$$
V_{1}(x, y)-\delta(y-x) \int_{0}^{1} d z V_{1}(z, y)
$$

type of relations are called "plus-prescription", see e.g. [8], where it was used for DGLAP equations. It satisfies

$$
\int_{0}^{1}[V(x, y)]_{+} d x=0
$$




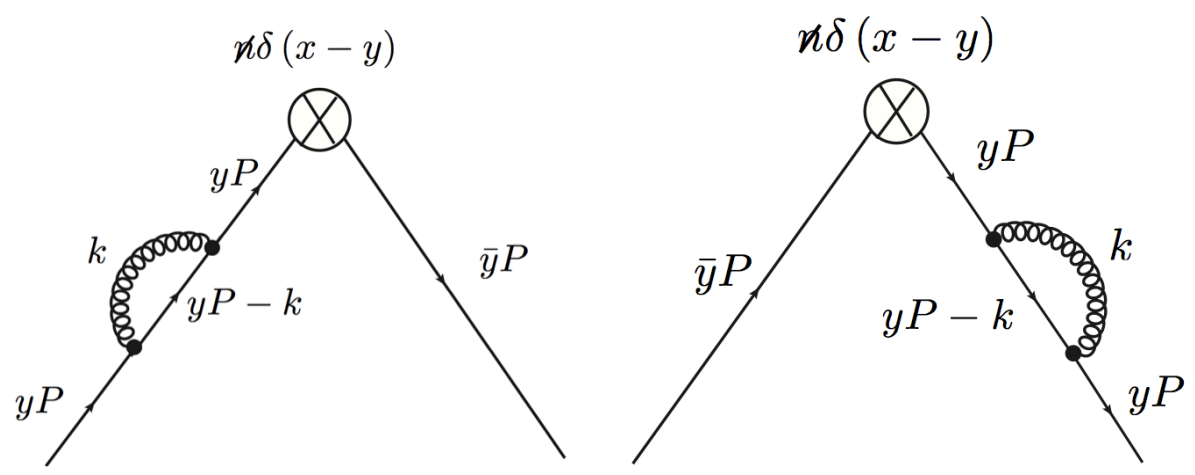

FIG. 7: Self energy diagram contributing LO ERBL kernel

This relation for $V(x, y)$ reflects the fact that the axial current is conserved for massless quarks, i.e. it is not renormalized.

In the leading logarithm approximation, the relevant ERBL evolution equation [1, 2] reads

$$
\frac{\partial \varphi(x, t)}{\partial t}=\int_{0}^{1}[V(x, y)]_{+} \varphi(y, t) d y,
$$

where $t=2 C_{F} \ln \ln (\mu / \Lambda) / b_{0}$ is the leading logarithm QCD evolution parameter,

$$
\begin{aligned}
V(x, y)= & {\left[\frac{x}{y}\left(1+\frac{1}{y-x}\right)\right] \theta(x<y) } \\
& +\left[\frac{\bar{x}}{\bar{y}}\left(1+\frac{1}{x-y}\right)\right] \theta(y<x)
\end{aligned}
$$

is the evolution kernel (we use $\bar{x}=1-x$ and $\bar{y}=1-y$ ), which has a singularity for $x=y$ regulated by the plus prescription

$$
[V(x, y)]_{+}=V(x, y)-\delta(y-x) \int_{0}^{1} V(z, y) d z,
$$

with respect to the first argument $x$.

In general, DAs $\varphi_{\pi}(x, \mu)$ depend on the normalization scale $\mu$, tending to the "asymptotic" shape,

$$
\varphi_{\pi}^{\mathrm{as}}(x)=6 f_{\pi} x(1-x),
$$


in the $\mu \rightarrow \infty$ limit, for any intial pion DA. A standard way [1, 2] to this result is to expand the initial pion DA over the eigenfunctions

$$
\varphi(x, \mu)=x(1-x) \sum_{n=0}^{\infty} C_{n}^{3 / 2}(2 x-1) k_{n}(\mu)
$$

of the evolution kernel. Each Gegenbauer projection then changes as $\left[\ln \ln \left(\mu_{0} / \Lambda\right) / \ln \ln (\mu / \Lambda)\right]^{\gamma_{n} / \beta_{0}}$ when $\mu$ increases. All anomalous dimensions $\lambda_{n}$ are positive, except for $\lambda_{0}$ which is zero, hence only the $\sim x(1-x)$ part survives for $t \rightarrow \infty$. For a pion DA given by a sum of a few Gegenbauer polynomials, this method gives a convenient analytic expression for the DA evolution. However, if the initial DA does not vanish at the end points, or has jumps inside the support region, one should formally take an infinite number of Gegenbauer polynomials. In practice, this means that one should sum over a large number of terms to get a reasonably precise (point by point) result for the evolved DA.

Another way to handle evolution of singular DA is the method we are going to describe in this thesis. To begin with, we need to do some rearrangements to the evolution equation. In explicit form the evolution equation is

$$
\frac{\partial \varphi(x, t)}{\partial t}=\int_{0}^{1}[V(x, y) \varphi(y, t)-V(y, x) \varphi(x, t)] d y
$$

It is clear that the singularities of $V(x, y)$ and $V(y, x)$ at $x=y$ cancel each other in the integrand above, even though the subtraction there does not look like a "+"-prescription with respect to the integration variable. Adding and subtracting $V(x, y) \varphi(x)$ in the integrand, we obtain the equation

$$
\frac{\partial \varphi(x, t)}{\partial t}=\int_{0}^{1} V(x, y)[\varphi(y, t)-\varphi(x, t)] d y-\varphi(x, t) \int_{0}^{1}[V(y, x)-V(x, y)] d y
$$

in which the first term has the structure of the "+"-prescription with respect to the integration variable, so that $1 /(x-y)$ singularity of $V(x, y)$ is canceled by zero of $\varphi(y, t)-\varphi(x, t)$ at $x=y$. The integral in the second term in Eq. 42

$$
v(x)=-\int_{0}^{1}[V(y, x)-V(x, y)] d y
$$

is also finite. Taking the Ansatz

$$
\varphi(x, t)=e^{t v(x)} \Phi(x, t)
$$


we obtain for $\Phi(x, t)$ the equation

$$
\frac{\partial \Phi(x, t)}{\partial t}=\int_{0}^{1} V(x, y)\left[e^{t[v(y)-v(x)]} \Phi(y, t)-\Phi(x, t)\right] d y
$$

which does not have the second term. The solution for $\Phi(x, t)$ may be written as a series in $t$

$$
\Phi(x, t)=\sum_{n=0}^{\infty} \frac{t^{n}}{n !} \Phi_{n}(x)
$$

with the functions $\Phi_{n}(x)$ satisfying the recurrence relation

$$
\Phi_{n+1}(x)=\int_{0}^{1} V(x, y)\left[\sum_{l=0}^{n} \frac{n !}{(n-l) ! l !} \Phi_{l}(y)[v(y)-v(x)]^{n-l}-\Phi_{n}(x)\right] d y .
$$

\subsection{DISTRIBUTION AMPLITUDES (DA) AND THEIR EVOLUTION WITH GEGENBAUER EXPANSION:}

One of the most important applications of pQCD is to predict the asymptotic behavior of hadronic form factors. The distribution amplitude $\varphi\left(x_{1}, x_{2}, \ldots, x_{n}, Q^{2}\right)$ is a function related to the hadron's wave function and describes how the longitudinal momentum of a fast-moving hadron is shared among constituents belonging to a particular Fock component. The reason for the $Q^{2}$ dependence is that all the soft gluon contributions of the form $\left(\alpha_{s} \ln \left(Q^{2} / m\right)^{2}\right)^{n}$ are factorized into the DA. This dependence of DA on $Q^{2}$ is governed by the ERBL evolution equation (36).

The standard way of calculating evolution of the DA is expanding the initial DA $\varphi\left(x, Q_{0}\right)$ over the eigenfunctions of the evolution kernel $V(x, y)$

$$
\int_{0}^{1} C_{n}^{3 / 2}(2 x-1) V(x, y) d x=-\gamma_{n} C_{n}^{3 / 2}(y)
$$

where $\gamma_{n}$ is non-singlet anomalous dimension given by

$$
\gamma_{n}=C_{F}\left[1-\frac{2}{(n+1)(n+2)}+4 \sum_{j=2}^{n+1} \frac{1}{j}\right]
$$

with $C_{F}$ being $\left(n_{c}^{2}-1\right) / 2 n_{c}=4 / 3$ and $n_{c}$ being number of colors. The general solution for the evolution equation in terms of Gegenbauer polynomials is

$$
\varphi\left(x_{i}, Q\right)=x_{1} x_{2} \sum_{n=0}^{\infty} k_{n}\left(Q^{2}\right) C_{n}^{3 / 2}\left(x_{1}-x_{2}\right)
$$


with the $k_{n}\left(Q^{2}\right)$ dependence on $Q^{2}$ given by

$$
k_{n}\left(Q^{2}\right)=e^{\gamma_{n} t} k_{n}\left(Q_{0}^{2}\right)=k_{n}\left(Q_{0}^{2}\right)\left(\frac{\ln \left(Q_{0}^{2} / \Lambda^{2}\right)}{\ln \left(Q^{2} / \Lambda^{2}\right)}\right)^{\gamma_{n} / \beta_{0}} .
$$

The orthogonality relation of Gegenbauer polynomials $C_{n}^{3 / 2}$ is given by,

$$
\int_{-1}^{1} d \xi\left(1-\xi^{2}\right) C_{n}^{3 / 2}(\xi) C_{m}^{3 / 2}(\xi)=\delta_{n, m} \frac{(n+2)(n+1)}{2(2 n+3)}
$$

Hence, the Gegenbauer polynomials $C_{n}^{3 / 2}(2 x-1)$ form an orthogonal and complete basis with the weight $x(1-x)$ and Eq. (52) can be written with the integral limits form 0 to 1 with the change of variable $\xi=2 x-1$,

$$
\int_{0}^{1} x \bar{x} C_{n}^{3 / 2}(2 x-1) C_{m}^{3 / 2}(2 x-1) d x=\delta_{n, m} \frac{(n+2)(n+1)}{4(2 n+3)} .
$$

This orthogonality relation can be used to extract the coefficients $k_{n}$,

$$
k_{n}\left(Q^{2}\right)=\frac{4(2 n+3)}{(n+1)(n+2)} \int_{0}^{1} d x C_{n}^{3 / 2}(2 x-1) \varphi\left(x, Q^{2}\right) .
$$

The general solution becomes

$$
\begin{aligned}
\varphi\left(x, Q^{2}\right) & =x \bar{x} \sum_{n=0}^{\infty} e^{-t \gamma_{n}} C_{n}^{3 / 2}(2 x-1) k_{n}\left(Q_{0}^{2}\right) \\
& =x \bar{x} \sum_{n=0}^{\infty}\left[\alpha_{s}\left(Q^{2}\right) / \alpha_{s}\left(Q_{0}^{2}\right)\right]^{\gamma_{n} / \beta_{0}} C_{n}^{3 / 2}(2 x-1) k_{n}\left(Q_{0}^{2}\right) .
\end{aligned}
$$

The meaning of this equation is that if the anomalous dimension is greater than zero, then the terms die off as powers of $\alpha_{s}\left(Q^{2}\right) / \alpha_{s}\left(Q_{0}^{2}\right)=\ln \left(Q_{0}^{2} / \Lambda^{2}\right) / \ln \left(Q^{2} / \Lambda^{2}\right)$. This means that for large $Q^{2}$, the DA approaches the first term of the expansion, i.e., its asymptotic shape $x(1-x)$. The expansion in Eq. (55) converges if $\varphi\left(x, Q^{2}\right)$ vanishes at the endpoints of interval $[0,1]$. In the next chapter, application of the Gegenbauer expansion method is illustrated on flat and anti-symmetric distribution amplitudes. The comparison between the output of Gegenbauer polynomial expansion and our analytic method is also demonstrated. 


\section{CHAPTER 3}

\section{EVOLUTION OF FLAT DA}

One of the motivations to study a flat DA $\varphi_{0}^{F}(x)=1$ is that it is the simplest example of a function that does not vanish at the end points $x=0$ and $x=1$. Another motivation for choosing a flat DA is BaBar data [30] on the $\gamma^{*} \gamma \rightarrow \pi^{0}$ transition form factor correspond to approximately logarithmic $\ln Q^{2}$ raise of the combination $Q^{2} F_{\gamma^{*} \gamma \pi^{0}}\left(Q^{2}\right)$ in the region of very high momentum transfers 10 to 40 $\mathrm{GeV}^{2}$, where the perturbative QCD approach [2] predicts nearly constant behavior for this combination. It was proposed [31, 32] to explain the BaBar "puzzle" by assuming that the pion distribution amplitude is "flat": $\varphi_{\pi}(x)=f_{\pi}$.

First, we study the evolution of a flat DA with the Gegenbauer expansion. One may check the expansion of a flat function in terms of Gegenbauer polynomials before turning the evolution on to illustrate the efficiency of this method on a function that does not vanish at the end points,

$$
1=x(1-x) \sum_{n=0, \text { even }}^{\infty} \frac{4(2 n+3)}{(n+1)(n+2)} C_{n}^{3 / 2}(2 x-1)
$$
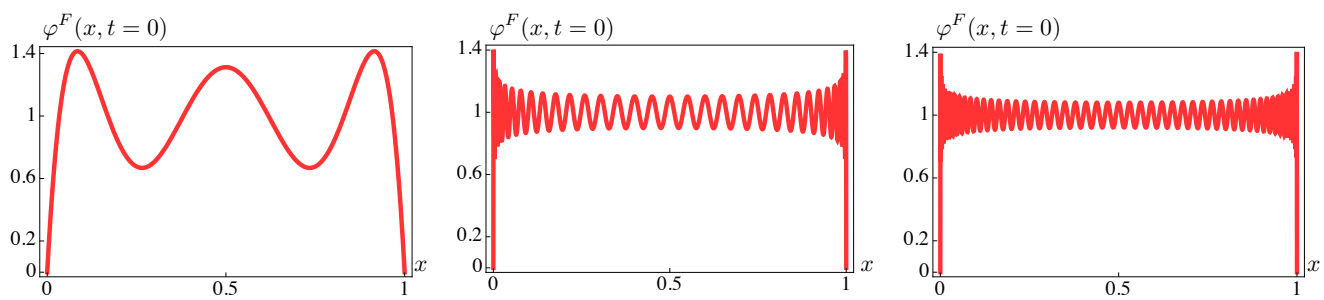

FIG. 8: Expansion of flat distribution amplitude in terms of Gegenbauer polynomials: 3 terms, 30 terms and 50 terms.

Fig. 8 shows that Gegenbauer polynomial expansion needs many terms to work for a function which does not vanish at end points $x=0$ and $x=1$ even before the evolution is turned on. Despite the fact that the expansion was not an efficient method for expanding a flat distribution, one may check the method on the evolution 
of a flat DA. All anomalous dimensions are positive, $\gamma_{n}>0$ except the first one which is $\gamma_{0}=0$. The first few anomalous dimensions for $\varphi_{0}^{F}(x)=1$ are

$$
\gamma_{0}=0, \gamma_{2}=\frac{50}{9}, \gamma_{4}=\frac{364}{45} \cdots
$$

This means that for large $Q^{2}$, a flat DA is going to evolve into the first term in the expansion which is $x \bar{x}$. The coefficients $k_{n}(t=0)$ are calculated with Eq. (54). Only terms with $n=$ even survive. The first few terms are equal to

$$
\begin{aligned}
& k_{0}(t=0)=6 \int_{0}^{1} d x C_{0}^{3 / 2}(2 x-1)=6, \\
& k_{2}(t=0)=\frac{7}{3} \int_{0}^{1} d x C_{2}^{3 / 2}(2 x-1)=\frac{7}{3}, \\
& k_{4}(t=0)=\frac{22}{15} \int_{0}^{1} d x C_{4}^{3 / 2}(2 x-1)=\frac{22}{15}, \cdots
\end{aligned}
$$

After calculating $k_{n}\left(Q_{0}^{2}\right)$, one can evaluate the DA to a different $Q^{2}$ by using Eq. (55), i.e. the evolution of DA.
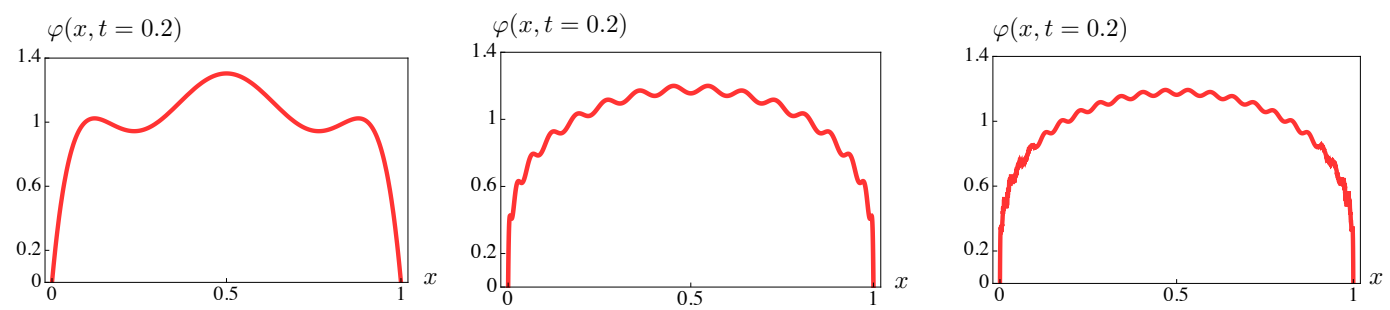

FIG. 9: Flat function expanded in terms of Gegenbauer polynomials: 2 terms, 30 terms and 50 terms.

As seen in Fig. 9, the Gegenbauer expansion produces functions which are oscillating. However, there is no physical reason for these structures. They only appear because of the nature of Gegenbauer polynomials, which was also illustrated for the flat DA before the evolution was turned on. In order to get the expected smooth function, one needs to sum a large amount of terms. The necessity for summation of a large amount of terms, even for the simplest singular DA, suggests that one needs a more efficient analytic method for singular functions. In the next section, we present an analytic method which provides the desired results with one or two iterations. 


\subsection{SINGULAR PART}

It is instructive to consider first an auxiliary situation when the evolution kernel is given by the singular part only

$$
V^{\text {sing }}(x, y)=\left(\frac{x}{y(y-x)}\right) \theta(x<y)+(x \rightarrow \bar{x}, y \rightarrow \bar{y})
$$

of the QCD kernel. In this case

$$
v^{\operatorname{sing}}(x) \equiv-\int_{0}^{1}\left[V^{\operatorname{sing}}(y, x)-V^{\operatorname{sing}}(x, y)\right] d y=2+\ln (x \bar{x})
$$

and the recurrence relation is given by

$$
\Phi_{n+1}(x)=\int_{0}^{1} V(x, y)\left[\sum_{l=0}^{n} \frac{n !}{(n-l) ! l !} \Phi_{l}(y)\left(\ln \frac{y \bar{y}}{x \bar{x}}\right)^{n-l}-\Phi_{n}(x)\right] d y,
$$

or, explicitly for the first terms,

$$
\begin{aligned}
\Phi_{1}(x) & =\int_{0}^{1} V(x, y)\left[\Phi_{0}(y)-\Phi_{0}(x)\right] d y \\
\Phi_{2}(x) & =\int_{0}^{1} V(x, y)\left[\Phi_{0}(y) \ln \left(\frac{y \bar{y}}{x \bar{x}}\right)+\Phi_{1}(y)-\Phi_{1}(x)\right] d y \\
\Phi_{3}(x) & =\int_{0}^{1} V(x, y)\left[\Phi_{0}(y) \ln ^{2}\left(\frac{y \bar{y}}{x \bar{x}}\right)+2 \Phi_{1}(y) \ln \left(\frac{y \bar{y}}{x \bar{x}}\right)+\Phi_{2}(y)-\Phi_{2}(x)\right] d y
\end{aligned}
$$

In this approximation, we can write

$$
\varphi^{\operatorname{sing}}(x, t)=(x \bar{x})^{t} e^{2 t}\left(\Phi_{0}(x)+t \Phi_{1}(x)+\frac{t^{2}}{2 !} \Phi_{2}(x)+\frac{t^{3}}{3 !} \Phi_{3}(x)+\ldots\right) .
$$

If we take the flat DA for $t=0$, i.e., $\Phi(x, 0)=1$, this gives

$$
\begin{aligned}
\Phi_{0}(x)= & 1 \\
\Phi_{1}(x)= & 0 \\
\Phi_{2}(x)= & -2 \ln x \ln \bar{x} \\
\Phi_{3}(x)= & 3 \ln (x \bar{x}) \ln x \ln \bar{x}+2\left[\ln x \operatorname{Li}_{2}(x)+\ln \bar{x} \operatorname{Li}_{2}(\bar{x})\right] \\
& -4\left[\operatorname{Li}_{3}(x)+\operatorname{Li}_{3}(\bar{x})\right]+8 \zeta(3)
\end{aligned}
$$

The graphical results for the expansion components are given in Fig. 10 .

As far as

$$
\int_{0}^{1}[V(x, y)]_{+} d x=0
$$



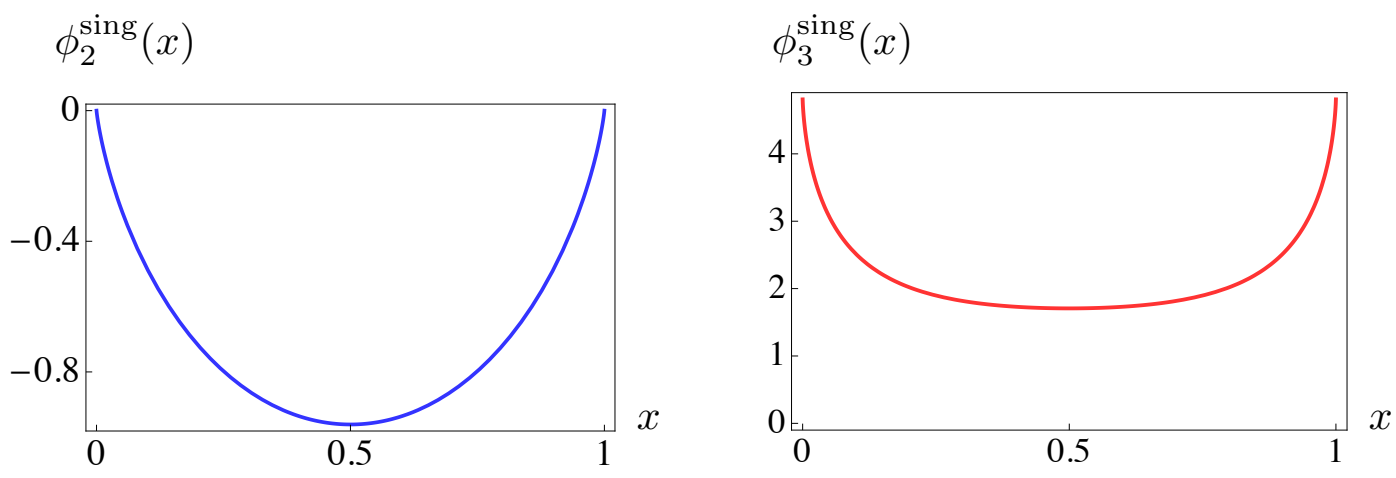

FIG. 10: Expansion components $\Phi_{2}^{\text {sing }}(x)$ and $\Phi_{3}^{\text {sing }}(x)$.

evolution does not change the normalization integral for $\varphi(x, t)$. In particular, if we expand $\varphi(x, t)$ in $t$

$$
\varphi(x, t)=\sum_{n=0}^{\infty} \varphi_{n}(x) \frac{t^{n}}{n !},
$$

we should have

$$
\int_{0}^{1} \varphi_{n}(x) d x=\delta_{n 0}
$$

However, when we take the singular kernel case Ansatz

$$
\varphi^{\operatorname{sing}}(x, t)=e^{t(2+\ln (x \bar{x}))} \Phi(x, t),
$$

the $(t \ln x \bar{x})^{N}$ terms are summed to all orders, while the series over $\varphi_{n}(x)$ is restricted to some finite order $N$. As a result, the approximates $\varphi_{(N)}(x, t)$ are not normalized to 1 . In particular, if we keep the terms up to $\Phi_{2}(x)$, the normalization integral is given by

$$
I_{2}(t) \equiv \int_{0}^{1} \varphi_{(2)}^{\text {sing }}(x, t) d x=e^{2 t} \frac{\left.\Gamma^{2}(1+t)\right)}{\Gamma(2+2 t)}\left\{1-t^{2}\left[\left(H_{t}-H_{1+2 t}\right)^{2}-\psi_{1}(2+2 t)\right]\right\}
$$

with $H_{n}$ being harmonic numbers and $\psi_{k}$ the polygamma function. One can check that $I_{2}(t)=1+\mathcal{O}\left(t^{3}\right)$. For the next approximation, i.e., for $\varphi_{(3)}^{\text {sing }}(x, t)$, the normalization integral is $I_{3}(t)=1+\mathcal{O}\left(t^{4}\right)$, etc. For $\varphi_{(N \rightarrow \infty)}(x, t)$, the normalization integral $I_{N}(t)$ will tend to 1 for all $t$. 
The normalization integrals versus $t$ are shown in Fig. 11. For approximations involving $\Phi_{0}(x)$ and $\Phi_{2}(x)$, the calculations were done analytically, while the curve corresponding to inclusion of $\Phi_{3}^{\text {sing }}(x, t)$ was calculated numerically. As seen from Fig. 11, adding more terms brings the normalization closer to 1.

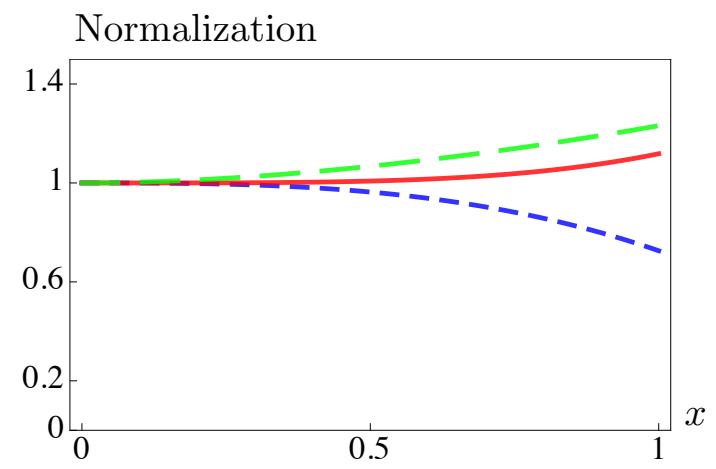

FIG. 11: Normalization factor calculated for terms including only $\Phi_{0}(x)$ (shortdashed line), $\Phi_{0}(x)$ and $\Phi_{2}(x)$ (long-dashed line) and $\Phi_{0}(x), \Phi_{2}(x)$ and $\Phi_{3}(x)$ (solid line).

In this situation, it makes sense to introduce the "normalized Ansatz", in which $\varphi(x, t)$ is approximated by the ratio

$$
\nu_{N}(x, t) \equiv \varphi_{(N)}(x, t) / I_{N}(t)
$$

so that the correct normalization of the $N$ th approximant is guaranteed for all $t$. In particular, this gives

$$
\nu_{2}(x, t)=(x \bar{x})^{t} \frac{\Gamma(2+2 t)}{\Gamma^{2}(1+t)} \frac{1-t^{2} \ln x \ln \bar{x}}{1-t^{2}\left[\left(H_{t}-H_{1+2 t}\right)^{2}-\psi_{1}(2+2 t)\right]} .
$$

As seen from this formula (and also from Fig, 12 , the initial flat function immediately (for whatever small positive $t$ ) evolves into a function vanishing at the end points with its shape dominated by the $(x \bar{x})^{t}$ factor. 


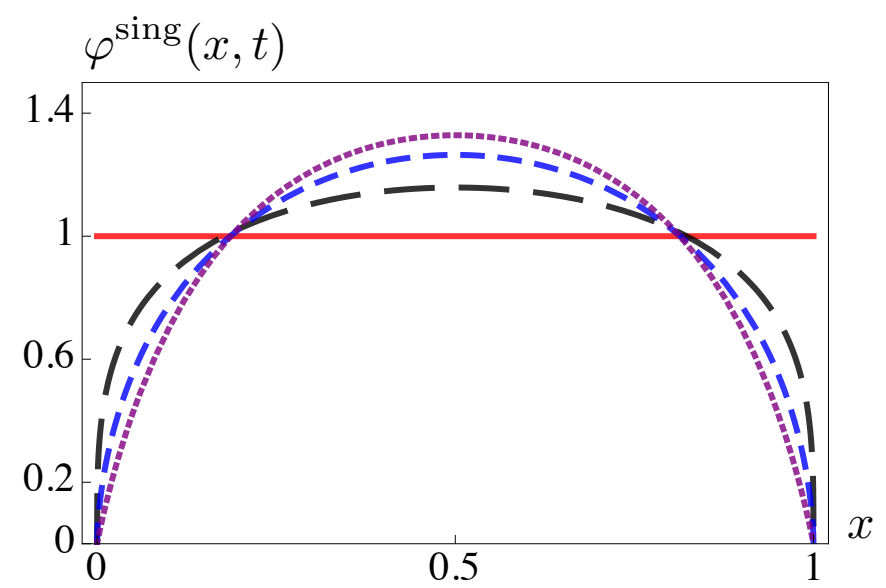

FIG. 12: Evolution of the flat DA under the singular part of the evolution kernel: the curves shown correspond to $t=0$ (red), $t=0.3$ (black), $t=0.6$ (blue), $t=1.0$ (purple).

\subsection{ADDING NON-SINGULAR PART}

When the whole QCD evolution kernel is taken into account, we have

$$
\begin{aligned}
v(x) \equiv-\int_{0}^{1}[V(y, x)-V(x, y)] d y & =3 / 2+x \ln \bar{x}+\bar{x} \ln x \\
& =v^{\operatorname{sing}}(x)-1 / 2-x \ln x-\bar{x} \ln \bar{x}
\end{aligned}
$$

Following the same steps as in the previous section, we calculate the expansion terms for the initial flat distribution amplitude:

$$
\begin{aligned}
\Phi_{0}(x)= & 1 \\
\Phi_{1}(x)= & 0 \\
\Phi_{2}(x)= & x \ln x[1+(x-1 / 2) \ln x]+\bar{x} \ln \bar{x}[1+(x+1 / 2) \ln \bar{x}]-\ln x \ln \bar{x} \\
& +x \operatorname{Li}_{2}\left(-\frac{\bar{x}}{x}\right)+\bar{x} \operatorname{Li}_{2}\left(-\frac{x}{\bar{x}}\right) .
\end{aligned}
$$

With these terms taken into account we have

$$
\varphi_{(2)}(x, t)=e^{3 t / 2}\left(x^{\bar{x}} \bar{x}^{x}\right)^{t}\left(1+\frac{t^{2}}{2 !} \Phi_{2}(x)\right) .
$$


Unfortunately, for this form it is impossible to analytically calculate the normalization integral even for the lowest term. Compared to the Ansatz used for the singular part of the evolution kernel, the Ansatz

$$
\varphi(x, t)=e^{t v(x)} \Phi(x, t)=e^{3 t / 2}\left(x^{\bar{x}} \bar{x}^{x}\right)^{t} \Phi(x, t)
$$

has an extra overall factor $\left[e^{-1 / 2} x^{-x} \bar{x}^{-\bar{x}}\right]^{t}$. Note that the function $x^{-x} \bar{x}^{-\bar{x}}$ is finite at the end points $x=0,1$, where it takes its minimal value for the interval $[0,1]$ (equal to 1 ), and has a maximum for $x=1 / 2$, where it equals 2 . Thus, the factor $e^{-1 / 2} x^{-x} \bar{x}^{-\bar{x}}$ enhances the $x \bar{x}$ profile in the middle (by a factor of $2 / \sqrt{e} \approx 1.2$ ) and suppresses it at the end points (by $1 / \sqrt{e} \approx 0.6$ ). This is a rather mild modification, and what is most important, it does not change the $\sim x^{t}$ (or $\sim \bar{x}^{t}$ ) behavior at the end points. So, it makes sense to use the expansion

$$
\left[x^{-x} \bar{x}^{-\bar{x}}\right]^{t}=\sum_{n=0}^{\infty}(-1)^{n}(x \ln x+\bar{x} \ln \bar{x})^{n} \frac{t^{n}}{n !},
$$

in powers of $t$ and combine it with the expansion for $\Phi(x, t)$. This corresponds to Ansatz

$$
\varphi(x, t)=(x \bar{x})^{t} e^{3 t / 2}\left(\tilde{\Phi}_{0}(x)+t \tilde{\Phi}_{1}(x)+\frac{t^{2}}{2 !} \tilde{\Phi}_{2}(x)+\ldots\right)
$$

whose expansion coefficients $\tilde{\Phi}_{n}(x)$ can be straightforwardly obtained from $\Phi_{n}(x)$ 's. In particular, $\tilde{\Phi}_{1}(x)=-(x \ln x+\bar{x} \ln \bar{x})$, and $\tilde{\Phi}_{2}(x)=\Phi_{2}(x)+(x \ln x+\bar{x} \ln \bar{x})^{2}$. The graphical results are shown in Fig. 13.
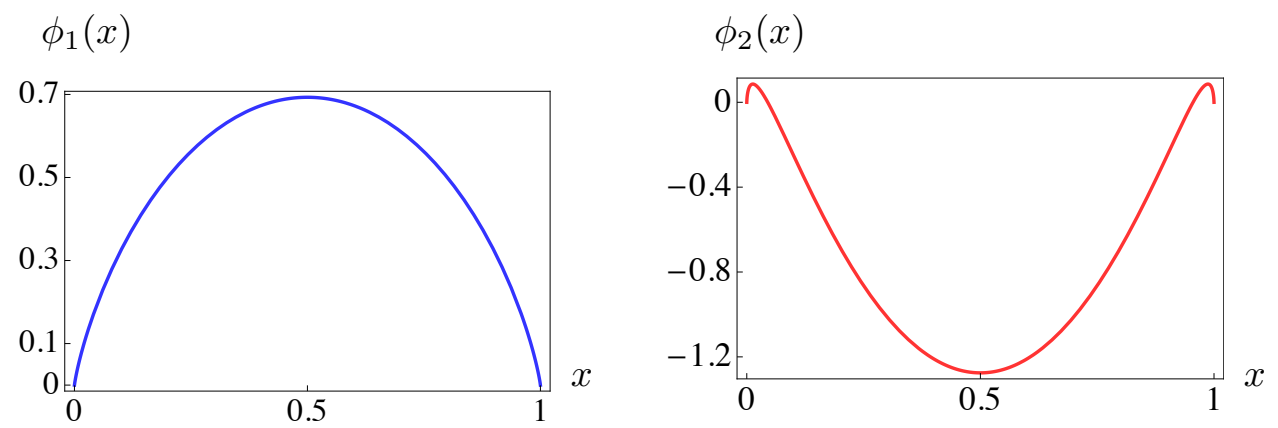

FIG. 13: Expansion components in the full kernel case: $\tilde{\Phi}_{1}(x)$ and $\tilde{\Phi}_{2}(x)$. 
Now, the normalization integral for the lowest terms can be calculated analytically:

$$
I_{1}(t) \equiv \int_{0}^{1} \varphi_{(1)}(x, t) d x=e^{3 t / 2} \frac{\Gamma^{2}(1+t)}{\Gamma(2+2 t)}\left(1-\frac{t}{2(t+1)}+\frac{t}{2}\left(-H_{t}+H_{1+2 t}\right)\right),
$$

where $H_{n}$ are harmonic numbers. Fig 14 a shows the normalization versus $t$.

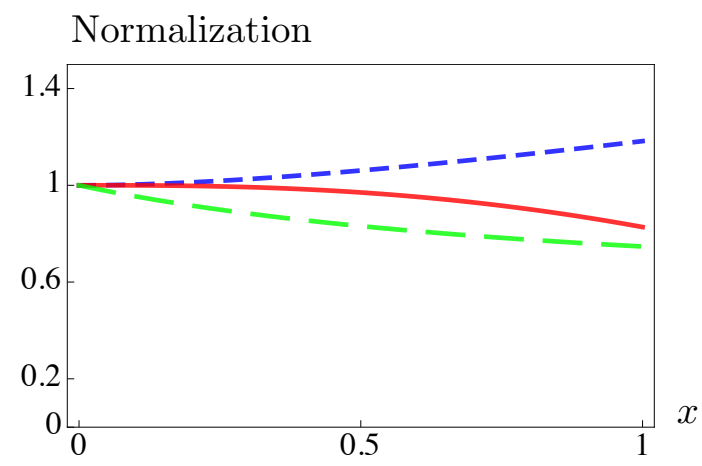

FIG. 14: a) Normalization factor calculated for terms including only $\Phi_{0}(x)$ (dotdashed line), $\Phi_{0}(x)$ and $\Phi_{2}(x)$ (dashed line) and $\Phi_{0}(x), \Phi_{2}(x)$ and $\Phi_{3}(x)$ (solid line).

Again, we may switch to the normalized Ansatz formed by the ratio $\varphi_{(N)}(x, t) / I_{N}(t)$. For a flat initial distribution, this gives

$$
\nu_{1}(x, t)=(x \bar{x})^{t} \frac{\Gamma(2+2 t)}{\Gamma^{2}(1+t)} \frac{1-t(x \ln x+\bar{x} \ln \bar{x})}{1-t / 2(t+1)+t\left(-H_{t}+H_{1+2 t}\right) / 2} .
$$

The evolution of the flat distribution amplitude with full kernel is shown in Fig. 15. 


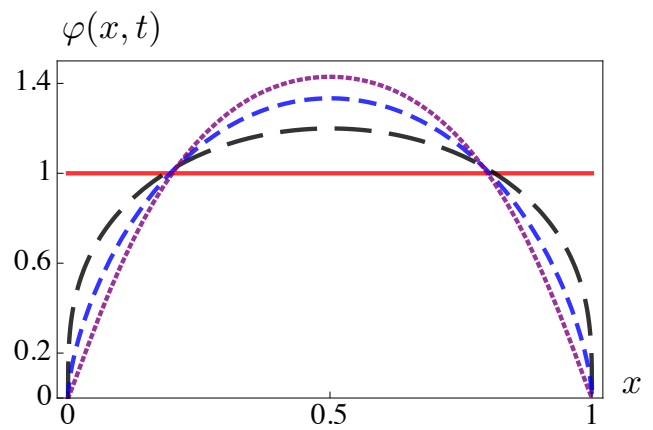

FIG. 15: Evolution of flat DA for the full kernel case: the curves shown correspond to $t=0$ (red), $t=0.3$ (black), $t=0.6$ (blue), $t=1.0$ (purple).

The comparison of this analytic method and the standard way of calculating the evolution, i.e., Gegenbauer expansion, is illustrated in Fig. 16. While with our method, one can achieve the results with only two iterations, the Gegenbauer expansion method still need more terms in addition to the fifty terms calculated.

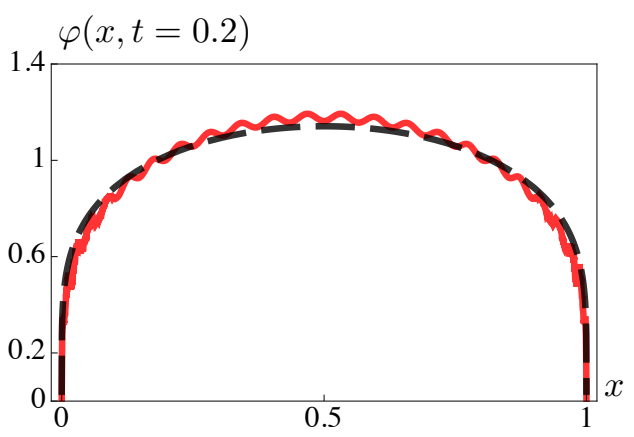

FIG. 16: Flat DA at $t=0.2$; The dashed curve is the analytic calculation and the solid curve is the Gegenbauer expansion with 50 terms. 


\section{CHAPTER 4}

\section{EVOLUTION OF ANTI-SYMMETRIC FLAT DA}

Evolution equations may be applied also in situations when the distribution amplitude is antisymmetric with respect to the change $x \rightarrow 1-x$. An interesting example is the $D$-term function $D(x)$ that appears in generalized parton distributions. Thus, let us consider the evolution of the DA that initially has the form

$$
\varphi_{0}(x)=\left\{\begin{array}{rl}
1 & 0<x \leqslant 1 / 2 \\
-1 & 1 / 2<x<1
\end{array}\right.
$$

Before applying our analytic method to this anti-symmetric DA, the application of the Gegenbauer expansion method should be studied. The first few coefficients in Eq. (54) gives the following few coefficients,

$$
\begin{aligned}
& k_{1}(t=0)=\frac{10}{3} \int_{0}^{1} d x C_{1}^{3 / 2}(2 x-1)=-5 \\
& k_{3}(t=0)=\frac{9}{5} \int_{0}^{1} d x C_{3}^{3 / 2}(2 x-1)=-\frac{9}{8} \\
& k_{5}(t=0)=\frac{26}{21} \int_{0}^{1} d x C_{5}^{3 / 2}(2 x-1)=-\frac{13}{8}, \cdots
\end{aligned}
$$

Once the coefficients $k_{n}(t=0)$ are known, one can calculate the DA at different $t$ by using Eq. (55), analogous to the flat DA case.
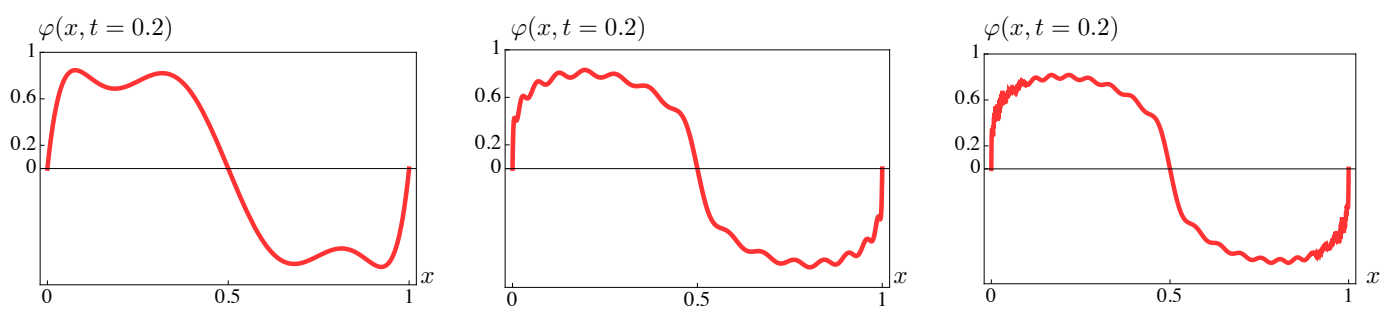

FIG. 17: Evolution of anti-symmetric DA at $t=0.2$ with the Gegenbauer expansion method: 2 terms, 30 terms and 50 terms.

Once again more terms in the summation lead to a smoother curve, but one needs a large amount of terms in the summation to get that smooth curve. Similar to the 
flat DA case, a smooth curve is the expected structure since there is no physical reason for the wiggles produced by Gegenbauer polynomials.

\subsection{SINGULAR PART}

Since we have demonstrated the reason why Gegenbauer expansion is not an effective method for the anti-symmetric DA, our analytic method can be applied to this case. The $v(x)$ function is the same because it depends only on kernel $V(x, y)$. Thus we can use the Ansatz (44) and expansion (46). Since $\varphi_{0}(x)$ is not just a constant, the first expansion coefficient $\Phi_{1}(x)$ is nonzero. Let us start with the singular part of the kernel. Then we get

$$
\Phi_{1}(x)=\left\{\begin{array}{cl}
-2 \ln \left[\frac{\bar{x}}{1-2 x}\right] & 0<x \leqslant 1 / 2, \\
2 \ln \left[\frac{x}{-1+2 x}\right] & 1 / 2<x \leqslant 1 .
\end{array}\right.
$$

We see that there are logarithmic terms $\ln |1-2 x|$ singular for $x=1 / 2$. These terms are natural, since each half of the antisymmetric DA on its interval is expected to evolve similarly to a flat DA on the $0 \leq x \leq 1$ interval. This observation suggests the Ansatz

$$
\varphi(x, t)=e^{2 t}(x \bar{x})^{t}|1-2 x|^{2 t} \Phi(x, t) .
$$

With this definition of $\Phi(x, t)$, the $\ln |1-2 x|$ terms are eliminated from $\Phi_{1}(x)$ :

$$
\Phi_{1}(x)=-2 \ln \bar{x} \theta(0<x \leqslant 1 / 2)-\{x \rightarrow \bar{x}\} .
$$

For the expansion component $\Phi_{2}(x)$, we have

$$
\begin{aligned}
\Phi_{2}(x)=\theta(0<x \leqslant 1 / 2) & \left\{-\frac{2 \pi^{2}}{3}+5 \ln ^{2} \bar{x}-2 \ln \bar{x} \ln x\right. \\
& +\ln (1-2 x)[4 \ln 2+4 \ln x-2 \ln \bar{x}+\ln (1-2 x)] \\
& -4 \operatorname{Li}_{2}(x)+4 \operatorname{Li}_{2}(2 x)+2 \operatorname{Li}_{2}\left[\frac{x}{\bar{x}}\right] \\
& \left.+2 \operatorname{Li}_{2}\left[\frac{x}{2 x-1}\right]+4 \operatorname{Li}_{2}\left[\frac{1-2 x}{\bar{x}}\right]\right\}-\{x \rightarrow \bar{x}\} .
\end{aligned}
$$

The graphical results for the expansion components are shown in Fig 18 .

The evolution of $\varphi_{\text {sing }}(x, t)$ to this accuracy can be obtained from

$$
\varphi(x, t)=e^{2 t}(x \bar{x})^{t}|1-2 x|^{2 t}\left(\varphi_{0}(x)+t \Phi_{1}(x)+\frac{t^{2}}{2 !} \Phi_{2}(x)\right) .
$$



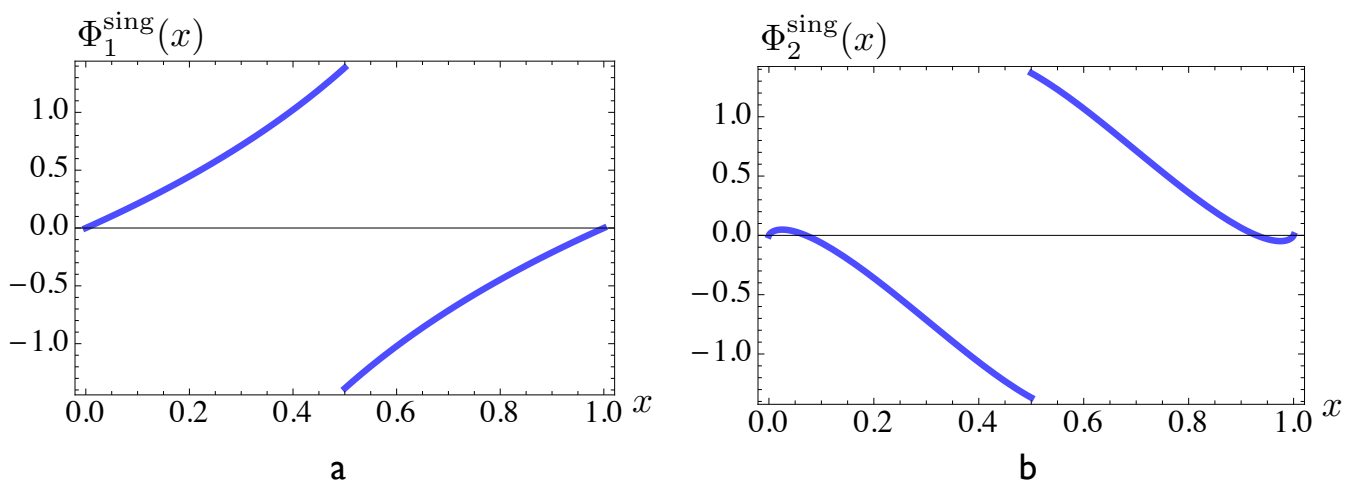

FIG. 18: Expansion coefficients for the antisymmetric DA: a) $\Phi_{1}^{\text {sing }}(x)$, b) $\Phi_{2}^{\text {sing }}(x)$.

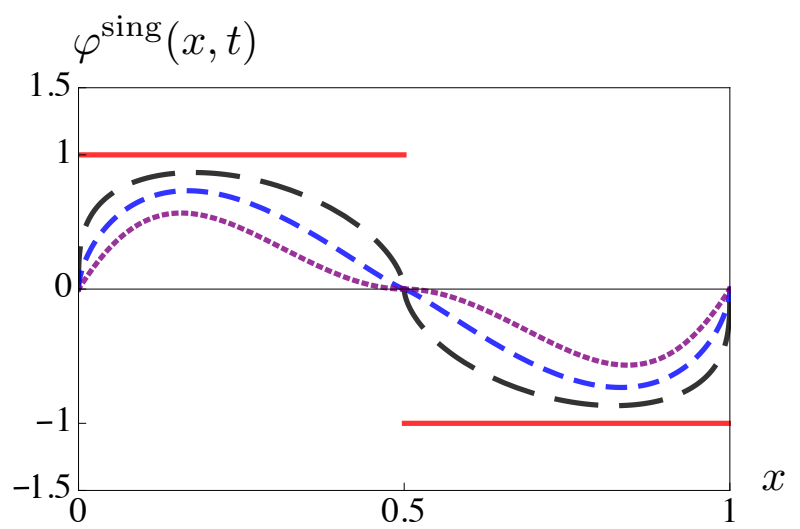

FIG. 19: Evolution of antisymmetric DA under the singular part of the kernel for $t=0, t=0.3, t=0.6, t=1$

As shown in Fig 19, the initial step function evolves into a function which is zero at the end points and in the middle point. 


\subsection{ADDING NON-SINGULAR PART OF THE KERNEL}

Since the nonsingular part does not add $\ln x$ and $\ln \bar{x}$ terms to $v(x)$, we may proceed with the same Ansatz

$$
\varphi(x, t)=e^{3 t / 2}(x \bar{x})^{t}|1-2 x|^{2 t} \Phi(x, t),
$$

but the expansion components change (see Fig. 20).
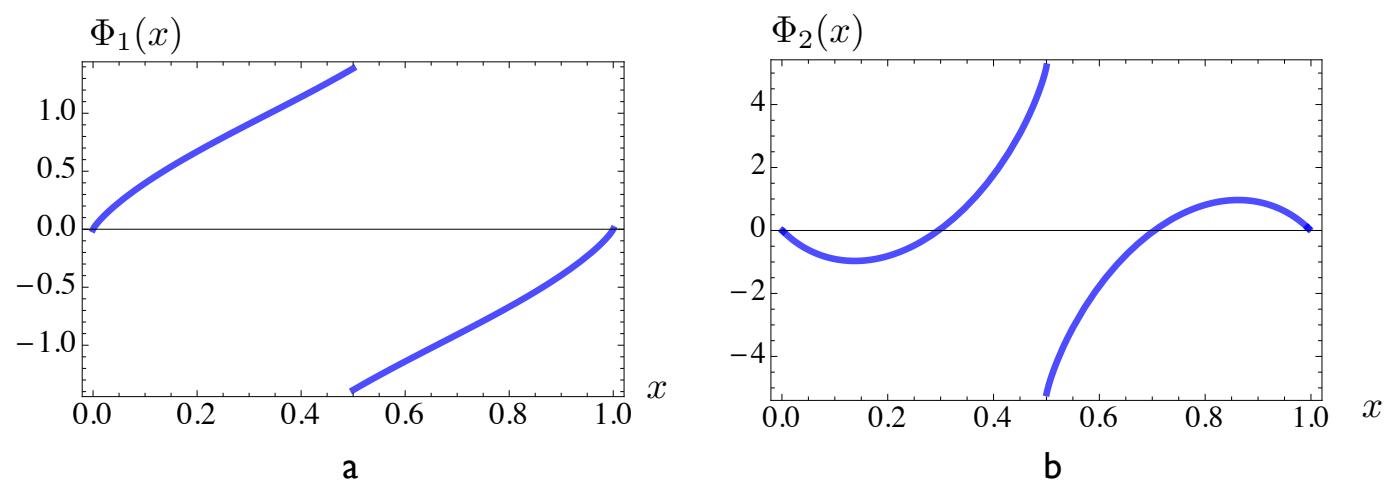

FIG. 20: Expansion components $\Phi_{1}(x)$ (a) and $\Phi_{2}(x)$ (b) in the case of the full kernel.

The distribution amplitude is now built using

$$
\varphi(x, t)=e^{3 / 2 t}(x \bar{x})^{t}|1-2 x|^{2 t}\left(\varphi_{0}(x)+t \Phi_{1}(x)+\frac{t^{2}}{2 !} \Phi_{2}(x)\right),
$$

For the first coefficient we have

$$
\Phi_{1}(x)=\theta(0<x \leqslant 1 / 2)\{-2 x \ln 2-2 \ln \bar{x}-(\bar{x} \ln \bar{x}+x \ln x)\}-\{x \rightarrow \bar{x}\},
$$

and for the second,

$$
\begin{aligned}
\Phi_{2}(x)=\theta(0<x \leqslant 1 / 2)\{ & -\frac{\pi^{2}}{2} \bar{x}+2 \ln ^{2}+2 x \ln 2+\ln \bar{x}[2+8 \ln 2+17 \ln \bar{x}] \\
& -(x-2 \ln (1-2 x)[\ln \bar{x}-\ln x]+2(5 x-3) \ln 2 \ln (1-2 x) \\
& -\ln ^{2}(1-2 x)-6 x \ln ^{2} \bar{x}+\ln \bar{x} \ln x \\
& +x \ln x(4 \ln 2+\ln \bar{x}+\ln x+2 \ln (1-2 x)+2 \ln \bar{x}) \\
& +2 \bar{x}\left[\operatorname{Li}_{2}\left(\frac{1-2 x}{2 \bar{x}}\right)+\operatorname{Li}_{2}\left(\frac{1-2 x}{\bar{x}}\right)+\operatorname{Li}_{2}(x)-\operatorname{Li}_{2}(2 x)\right] \\
& \left.-(1+2 x) \operatorname{Li}_{2}\left(-\frac{x}{\bar{x}}\right)+\operatorname{Li}_{2}\left(\frac{x^{2}}{\bar{x}^{2}}\right)-2 \operatorname{Li}_{2}\left(\frac{x}{2 x-1}\right)\right\} \\
& -\{x \rightarrow \bar{x}\} .
\end{aligned}
$$




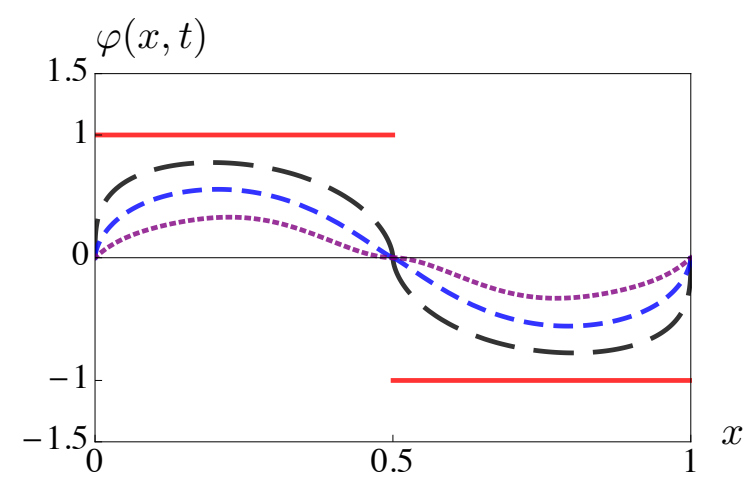

FIG. 21: Evolution of the antisymmetric DA in the full kernel case. The curves for $t=0$ (red), $t=0.3$ (black), $t=0.6$ (blue), $t=1.0$ (purple) are shown.

As may be seen from Fig.19, the resulting curves are rather close to those obtained when only the singular part of the kernel was taken into account. Thus, we observe that the $t^{n}$ series converges rather rapidly as far as $t \lesssim 1$. When $t \gtrsim 1$, the DAs is close to the asymptotic form, and one can switch to the solution in the form of the Gegenbauer expansion. The same comparison of this analytical method and Gegenbauer expansion done in for the flat DA is also done for the anti-symmetric case. The comparison is illustrated in Fig. 22

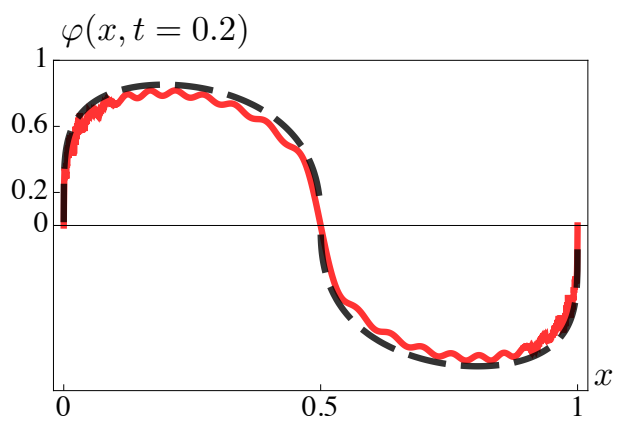

FIG. 22: Anti-symmetric DA at $t=0.2$; The dashed curve is the analytical calculation and the solid curve is the Gegenbauer expansion with 50 terms. 


\subsection{EVOLUTION OF JUMPS}

Another example of a singularity is given by DAs with a jump, the simplest case being

$$
\Phi_{0}^{J}(x, \zeta ; a, b)=\left\{\begin{array}{cc}
a & 0<x \leqslant \zeta \\
b & \zeta<x<1
\end{array}\right.
$$

The part of the first iteration $\Phi_{1}(x)$ generated by the singular part of the kernel

$$
\Phi_{1}^{J \operatorname{sing}}(x, \zeta ; a, b)=\left\{\begin{aligned}
(a-b) \ln \left[\frac{\zeta-x}{(1-x) \zeta}\right] & 0<x \leqslant \zeta \\
-(a-b) \ln \left[\frac{x-\zeta}{(1-\zeta) x}\right] & \zeta<x \leqslant 1
\end{aligned}\right.
$$

contains logarithmic terms $\ln |\zeta-x|$ singular for $x=\zeta$. Their structure may be understood in the following way. The original function $\varphi_{0}^{J}(x, \zeta ; a, b)$ may be represented as a sum of a constant $\frac{1}{2}(a+b)$ and a function $\frac{1}{2}(a-b) \operatorname{sign}(\zeta-x)$ that jumps by $b-a$ at the point $x=\zeta$. The constant part has no singularities at $x=\zeta$, so one can apply the original $\frac{1}{2}(a+b)(x \bar{x})^{t} \Phi$ Ansatz to it, while for the jumping part one may use the Ansatz

$$
\begin{aligned}
& \Phi^{J}(x, \zeta ; a, b ; t)=\frac{a-b}{2} e^{2 t}(x \bar{x})^{t}\left[\left(\frac{1-x / \zeta}{1-x}\right)^{2 t} \theta(x<\zeta)\right. \\
& \left.+\left(\frac{1-\bar{x} / \bar{\zeta}}{1-\bar{x}}\right)^{2 t} \theta(x>\zeta)\right] \Phi(x, \zeta)+\Psi(x, \zeta, t)
\end{aligned}
$$

The part containing square brackets is intended to take care of the evolution of the jump at $x=\zeta$. However, this part by construction vanishes at $x=\zeta$, while one would expect that evolution tends to convert $\varphi^{J}(x, \zeta ; a, b)$ into a universal $\zeta$-independent function proportional to $x \bar{x}$ or $x \bar{x}(1-2 x)$ (depending on the symmetry of the function). Thus, there should be also a part regular at the jump point. The function $\Psi(x, \zeta, t)$ is introduced to satisfy this requirement. It vanishes for $t=0$, but eventually becomes the dominant part.

Let us discuss a more general case, when a function has antisymmetric jumps at some locations $x=\zeta_{i}$. "Antisymmetric" means that the function approaches from opposite values on the sides of a jump, so that "on average" it is zero at the jump points. Then one can try the Ansatz

$$
\varphi(x, t)=\Phi(x, t)+\Psi(x, t)
$$


where

$$
\Phi(x, t)=e^{t[v(x)+w(x)]} \Phi_{0}(x),
$$

with the function $w(x) \sim \sum_{i} \ln \left|x-\zeta_{i}\right|$ intended to absorb major features of the evolution of the starting distribution $\varphi(x, t=0) \equiv \Phi_{0}(x)$ in the vicinity of the jump points, while the remainder $\Psi(x, t)$ is expected to be a regular function vanishing for $t=0$. As a result, we get the following equation:

$$
\begin{gathered}
\frac{\partial \Psi(x, t)}{\partial t}=\int_{0}^{1} V(x, y)[\Psi(y, t)-\Psi(x, t)] d y+v(x) \Psi(x, t) \\
+\int_{0}^{1} V(x, y)[\Phi(y, t)-\Phi(x, t)] d y-w(x) \Phi(x, t) .
\end{gathered}
$$

This is an inhomogeneous evolution equation for $\Psi(x, t)$, with starting condition $\Psi(x, t=0)=0$. For its derivative at $t=0$ we have

$$
\left.\frac{\partial \Psi(x, t)}{\partial t}\right|_{t=0}=\int_{0}^{1} V(x, y)\left[\Phi_{0}(y)-\Phi_{0}(x)\right] d y-w(x) \Phi_{0}(x) \equiv \chi(x) .
$$

To avoid singularities at the jump points, we should adjust $w(x)$ in such a way as to make $\chi(x)$ a continuous function of $x$. Then $\Psi(x, t) \approx t \chi(x)$ for small $t$. The corrections to this approximation can be found by iterations. Namely, we represent $\Psi(x, t)=\sum_{n=1} \Psi_{n}(x, t)$ and start with

$$
\Psi_{1}(x, t) \equiv \int_{0}^{t} d \tau\left\{\int_{0}^{1} V(x, y)[\Phi(y, \tau)-\Phi(x, \tau)] d y-w(x) \Phi(x, \tau)\right\}
$$

generating further terms using

$$
\Psi_{n+1}(x, t) \equiv \int_{0}^{t} d \tau\left\{\int_{0}^{1} V(x, y)\left[\Psi_{n}(y, \tau)-\Psi_{n}(x, \tau)\right] d y+v(x) \Psi_{n}(x, \tau)\right\}
$$

Since the derivative of $\Psi_{1}(x, t)$ for $t=0$ is given by $\chi(x)$, we can write

$$
\begin{aligned}
\Psi_{1}(x, t)= & t \chi(x)+\int_{0}^{t} d \tau \int_{0}^{1} V(x, y)[\delta \Phi(y, \tau)-\delta \Phi(x, \tau)] d y-w(x) \delta \Phi(x, \tau) \\
& \equiv t \chi(x)+\delta \Psi_{1}(x, t)
\end{aligned}
$$

Here, $\delta \Phi(x, \tau) \equiv \Phi(x, \tau)-\Phi_{0}(x)$ is the deviation of the Ansatz function $\Phi(x, \tau)$ from its $\tau=0$ shape. For small $\tau$, the function $\delta \Phi(x, \tau)$ has a rather sharp behavior at the jump points $x=\zeta_{i}$, and this results in a rather sharp behavior of $\delta \Psi_{1}(x, t)$ at these points. Since each iteration $\Psi_{n+1}(x, t)$ is generated linearly from a previous $\Psi_{n}(x, t)$ 
one (see Eq. (100) ), it makes sense to split $\Psi(x, t)$ into a "smooth" part $\Psi_{\chi}(x, t)$ generated by iterations of $t \chi(x)$ and the remainder $\delta \Psi(x, t)$ generated by iterations of $\delta \Psi_{1}(x, t)$. Thus, we have

$$
\varphi(x, t)=\Phi(x, t)+\delta \Psi(x, t)+\Psi_{\chi}(x, t)
$$

where the first two terms, $\Phi(x, t)$ and $\delta \Psi(x, t)$ have a rather sharp behavior at the jump points for small $t$, while $\Psi_{\chi}(x, t)$ has a smooth behavior.

In this chapter, we demonstrated our analytic method for general cases of singular DAs. Next chapter, we will illustrate the application of the method to the evolution of two-photon generalized distribution amplitude. 


\section{CHAPTER 5}

\section{STRUCTURE OF PHOTON GENERALIZED}

\section{DISTRIBUTION AMPLITUDE}

Using photons is a very effective tool for testing Quantum Chromodynamics (QCD). The kinematic possibilities provided by the two-photon state are plenty, so the twophoton state attracts attention not only in experimental studies but also in theoretical studies.

Due to these advantages of photon studies, the partonic structure of the photon has been discussed in lots of paper beginning with Ref. [33]. In Ref. [34], in order to describe the factorization of the non diagonal kinematics of Deeply Virtual Compton Scattering (DVCS), $\left(\gamma^{*} \gamma \rightarrow \gamma \gamma\right)$, Generalized Parton Distributions (GPDs) are used. GPDs are developed to explain one of the important problems of QCD, that is understanding the internal structure of hadrons. Generalized distribution amplitudes (GDAs) [16 18, 35, 36] which are related to GPDs by crossing, are non-perturbative objects which describe the transition from a quark-antiquark or a gluon-gluon pair into a hadron pair [17]. Two-meson GDAs are crossed-channel analogs of the meson Generalized Parton Distributions (GPDs). GDAs parametrize the matrix elements of light-cone operators between the vacuum and a system of hadrons, while GPDs parametrize the matrix element of the same operators between two different hadron states. The definition of the quark-antiquark GDA, for the most studied system $\left(\pi^{+} \pi^{-}\right)$[18], is

$$
\Phi_{q}^{\pi^{+} \pi^{-}}(z, \zeta)=\int \frac{d x^{-}}{2 \pi} e^{-i z P^{+} x^{-}}\left\langle\pi\left(p^{\prime}\right) \pi(p)\left|\bar{\psi}_{q}\left(x^{-}\right) \gamma^{+} \psi^{-} q(0)\right| 0\right\rangle
$$

Similar to two-meson GDAs, two-photon GDAs describe the transition of a quarkantiquark or a gluon-gluon pair into a photon pair. Analogous to the relation between two meson GDAs and the meson GPDs, two photon GDAs have the same crossing relation with photon GPDs.

Prediction of a GPD or GDA for any scale $\mu$ by using the measured ones at a scale $\mu^{\prime}$ is defined as evolution of GPDs or GDAs, where $\mu$ and $\mu^{\prime}$ are large enough 
so that $\alpha_{s}(\mu)$ and $\alpha_{s}\left(\mu^{\prime}\right)$ are small. This part of the study's focus is the evolution of the non-singlet vector two-photon GDA [18], which was calculated for the reaction $\gamma^{*}(q) \gamma\left(q^{\prime}\right) \rightarrow \gamma\left(p_{1}\right) \gamma\left(p_{2}\right)$. The evolution of the nonsinglet vector part of the diphoton GDA is governed by the ERBL evolution equation. The usual way, i.e. Gegenbauer expansion, was used in Ref. [37]. The result was calculated with a finite number of terms which have numerical instabilities around the points where initial GDA is discontinuous. In order to get a reliable result for the evolution of a non-singlet di-photon GDA, an infinite sum of Gegenbauer polynomials is needed.

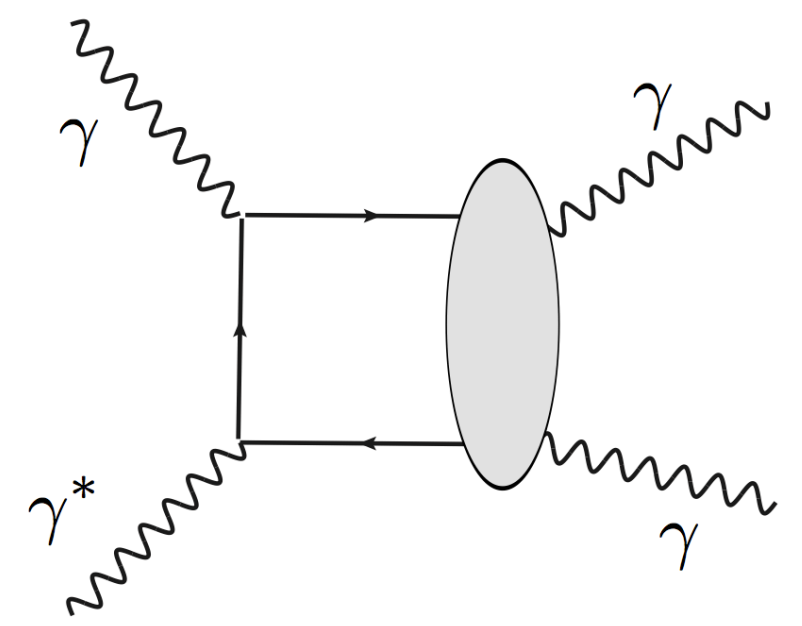

FIG. 23: Parton picture for the two-photon GDA

In the lowest QCD order, the non-singlet two-photon GDA is given by [38]

$$
\psi^{q}\left(x, \zeta, Q^{2}\right)=\frac{N_{C} e_{q}^{2}}{2 \pi^{2}} \log \frac{Q^{2}}{m^{2}} \varphi(x, \zeta),
$$

where the function $\varphi(x, \zeta)$ is proportional to the $V V \rightarrow V V$ component,

$$
\begin{aligned}
\varphi(x, \zeta) & =\frac{\bar{x}(2 x-\zeta)}{\bar{\zeta}} \theta(x-\zeta)+\frac{\bar{x}(2 x-\bar{\zeta})}{\zeta} \theta(x-\bar{\zeta}) \\
& -\frac{x(2 \bar{x}-\bar{\zeta})}{\zeta} \theta(\zeta-x)-\frac{x(2 \bar{x}-\zeta)}{\bar{\zeta}} \theta(\bar{\zeta}-x),
\end{aligned}
$$

of the ERBL evolution kernel matrix. QCD corrections induce further evolution of the photon GDA.

Namely, its derivative with respect to $\ln Q^{2}$ obeys the ERBL evolution equation with the $q q \rightarrow q q$ kernel considered above. In what follows, we study ERBL evolution 
of the function $\varphi(x, \zeta, t)$ which for the starting evolution point $t=0$ coincides with $\varphi(x, \zeta)$.

The function $\varphi(x, \zeta)$ is antisymmetric with respect to $x \leftrightarrow \bar{x}$ interchange and symmetric with respect to $\zeta \leftrightarrow \bar{\zeta}$ interchange. Thus, without loss of generality, we may choose $\zeta \leq 1 / 2$. Then $0 \leq \zeta \leq \bar{\zeta} \leq 1$, and it makes sense to explicitly write the function in each of the three regions:

$$
\begin{aligned}
\varphi(x, \zeta)= & \left\{\frac{x}{\zeta \bar{\zeta}}\left[\bar{\zeta}^{2}+\zeta^{2}-2 \bar{x}\right] \theta(0 \leq x \leq \zeta)-\frac{\zeta(1-2 x)}{1-\zeta} \theta(\zeta \leq x \leq \bar{\zeta})\right. \\
& \left.-\frac{\bar{x}}{\zeta \bar{\zeta}}\left[\bar{\zeta}^{2}+\zeta^{2}-2 x\right] \theta(\bar{\zeta} \leq x \leq 1)\right\} .
\end{aligned}
$$

The function is discontinuous at $x=\zeta$ and $x=\bar{\zeta}$.

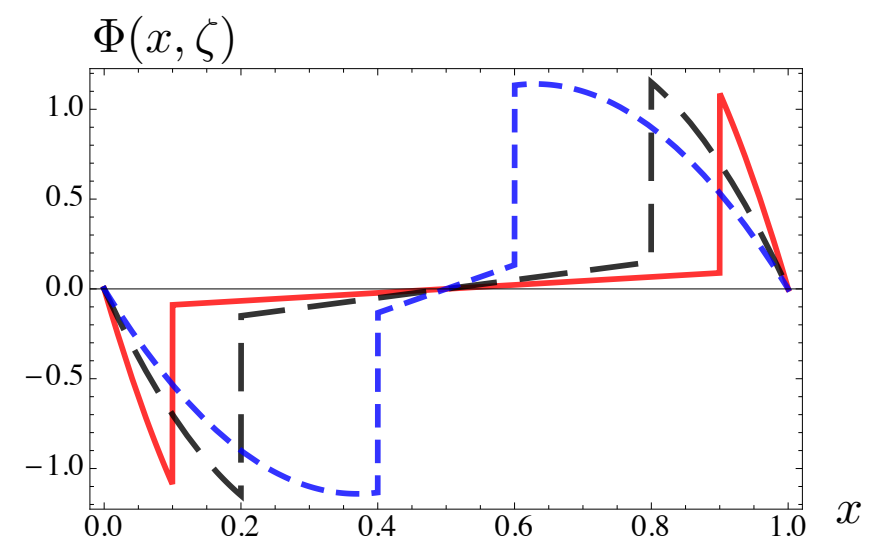

FIG. 24: Two-photon GDA profile function $\Phi(x, \zeta)$ at values $\zeta=0.1 ; 0.2 ; 0.4$.

Fig 24 shows the $x$-profile of the two-photon GDA at different $\zeta$ values. As $x$ approaches $\zeta$, the limiting value of the function from the left is $\varphi\left(\zeta_{-}, \zeta\right)=-1-\zeta \frac{1-2 \zeta}{1-\zeta}$, while from the right we have $\varphi\left(\zeta_{+}, \zeta\right)=-\zeta \frac{1-2 \zeta}{1-\zeta}$, so that the jump $\varphi\left(\zeta_{+}, \zeta\right)-$ $\varphi\left(\zeta_{-}, \zeta\right)=1$ is equal to 1 . According to our discussion in the preceding section, to treat the evolution of a jump, we should represent the initial function as a sum of a function $\varphi_{2}(x, \zeta)$ continuous in the vicinity of each jump, and a function $\varphi_{1}(x, \zeta)$ that has an antisymmetric jump of necessary size. The function $\varphi_{1}(x, \zeta)$ will also specify the initial form of the $\Phi$-part of the evolution Ansatz (95) for this function, so we will denote it as $\Phi_{1,0}(x, \zeta)$. For simplicity, we will choose it to be given by 
linear functions of $x$ in each of the three regions. As a result,

$$
\Phi_{1,0}(x, \zeta)=-\frac{x}{2 \zeta} \theta(0<x<\zeta)+\frac{\bar{x}}{2 \zeta} \theta(\bar{\zeta}<x<1)+\frac{1-2 x}{2(1-2 \zeta)} \theta(\zeta<x<\bar{\zeta}) .
$$

The function $\Phi_{1,0}(x, \zeta)$ is discontinuous at $x=\zeta$ and $x=\bar{\zeta}$, see Fig 25 a, where it is shown for $\zeta=0.2$. The function $\Phi_{2,0}(x, \zeta)$ specifying initial shape of the continuous part (see Fig $25 \mathrm{~b}$ ), is obtained as the difference between $\varphi(x, \zeta)$ and $\Phi_{1,0}(x, \zeta)$.
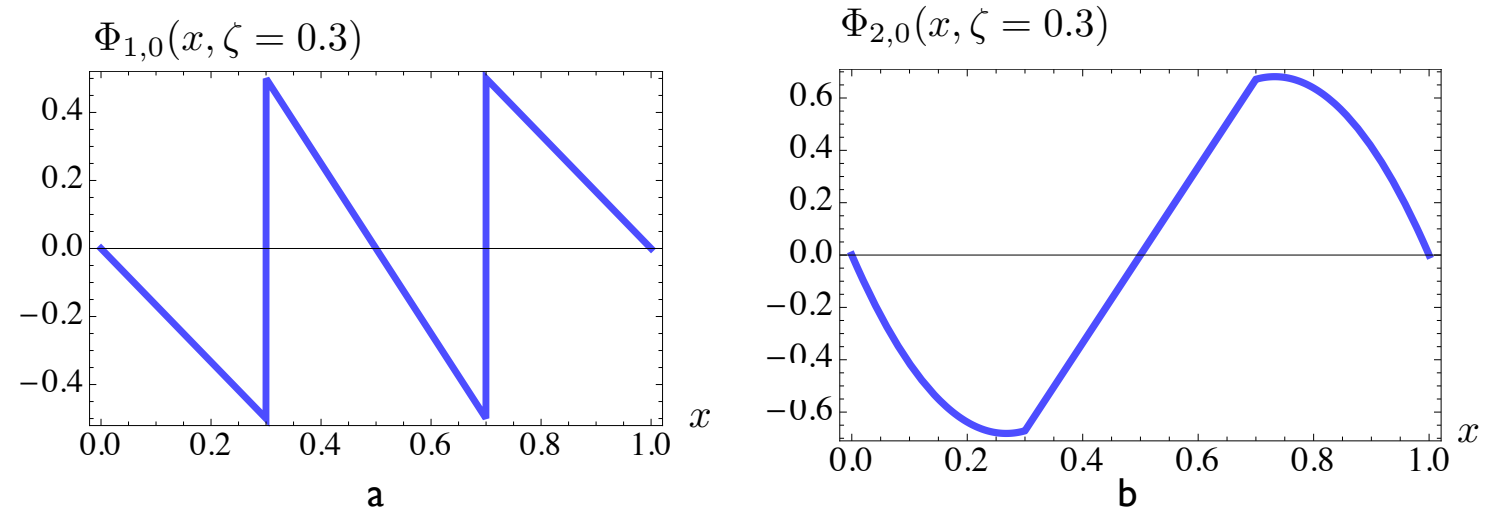

FIG. 25: (a) The $x$-profile of GDA $\Phi_{1,0}(x, \zeta)$ at $\zeta=0.3$. (b) Initial function $\Phi_{2,0}(x, \zeta)$ for $\zeta=0.3$.

In this chapter, we illustrated the two-photon generalized distribution amplitudes. We also introduced a separation to the two-photon GDA as jump part and cusp part. Next chapter, we demonstrate the evolution of the jump part. 


\section{CHAPTER 6}

\section{EVOLUTION OF THE JUMP PART OF THE TWO-PHOTON GDA}

In this chapter, we present the evolution of $\Phi_{1,0}(x, \zeta)$, i.e. jump part of two-photon GDA. As presented in Fig. 25a, $\Phi_{1,0}(x, \zeta)$ has jumps at $x=\zeta$ and $x=\bar{\zeta}$. Iteration of the initial function $\Phi_{1,0}(x, \zeta)$ with evolution kernel gives

$$
\begin{aligned}
\Phi_{1,1}(x, \zeta) \equiv & \int_{0}^{1} V(x, y)\left[\Phi_{1,0}(y, \zeta)-\Phi_{1,0}(x, \zeta)\right] d y \\
& =\theta(0<x<\zeta<1 / 2)\left\{(1-4 \zeta) \frac{x}{2 \zeta}+\frac{1}{2 \zeta}[x \bar{x} \ln x+(1-x \bar{x}) \ln \bar{x}]\right. \\
& -\ln (\zeta-x)-\ln (\bar{\zeta}-x)+\frac{\ln \zeta+\ln \bar{\zeta}(1-3 \zeta) / \zeta}{2(1-2 \zeta)} \bar{x} \\
& \left.-\frac{1-4 \zeta}{\zeta(1-2 \zeta)}[(x-\zeta) \ln (\zeta-x)+(\bar{\zeta}-x) \ln (\bar{\zeta}-x)]\right\}-\{x \rightarrow \bar{x}\} \\
& +\theta(\zeta<x<\bar{\zeta})\left\{\frac{1-2 x}{2(1-2 \zeta)}[(1-4 \zeta)+\ln \bar{\zeta}(1-3 \zeta) / \zeta]\right. \\
& +\ln (x-\zeta)-\ln (\bar{\zeta}-x) \\
& +\frac{1-4 \zeta}{2 \zeta(1-2 \zeta)}[(x-\zeta) \ln (x-\zeta)-(\bar{\zeta}-x) \ln (\bar{\zeta}-x)+\bar{x} \ln \bar{x}-x \ln x] \\
& \left.+\frac{x^{2}+\bar{x}^{2}}{2(1-2 \zeta)} \ln \left(\frac{\bar{x}}{x}\right)\right\} .
\end{aligned}
$$

As expected, $\Phi_{1,1}(x, \zeta)$ has logarithmic singularities

$$
-\ln (\zeta-x) \theta(x<\zeta)+\ln (x-\zeta) \theta(\zeta<x<1 / 2)-\{x \rightarrow \bar{x}\}
$$

for $x=\zeta$ and $x=\bar{\zeta}$ (see Fig. 26).

The sum of these terms may be written as $2(\ln |x-\zeta|+\ln |x-\bar{\zeta}|) \Phi_{1}(x, \zeta)$ plus regular terms, which suggests to take the Ansatz (95) with $w(x)$ containing $2(\ln \mid x-$ $\zeta|+\ln | x-\bar{\zeta} \mid)$. Namely, let us try the function $w_{0}(x, \zeta)$ given by

$$
w_{0}(x, \zeta)=4+2 \ln |x-\zeta|+2 \ln |x-\bar{\zeta}|
$$




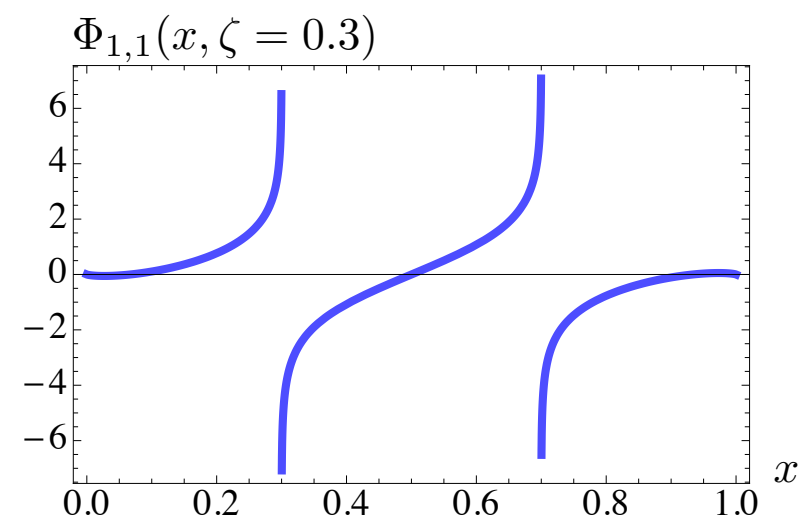

FIG. 26: First iteration $\Phi_{1,1}(x, \zeta)$ for $\zeta=0.3$.

The constant part " 4 " was chosen to make the integral of $w_{0}(x)$ closer to zero (it vanishes both for $\zeta=0$ and $\zeta=1$ ), i.e. to keep the overall normalization of the Ansatz factor closer to 1 . The resulting function (which gives the first term of the $\Psi$-part of the Ansatz (Eq. 95) ) is given by

$$
\Psi_{1,1}^{(0)}(x, \zeta)=\Phi_{1,1}(x, \zeta)-w_{0}(x, \zeta) \Phi_{1}(x, \zeta)
$$

and shown in Fig. 27.

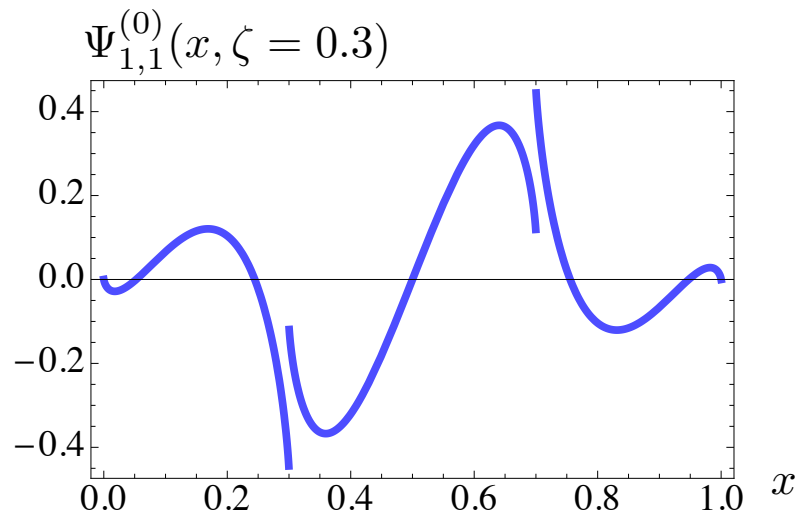

FIG. 27: Correction functions $\Psi_{1,1}^{(0)}(x, \zeta=0.3)$ with $w_{0}$ Ansatz.

One can see that, after the subtraction of singularities, we still have finite jumps 
for $x=\zeta$ and $x=\bar{\zeta}$. Explicit calculation gives

$$
\begin{aligned}
\Psi_{1,1}^{(0)}\left(\zeta_{+}, \zeta\right) & -\Psi_{1,1}^{(0)}\left(\zeta_{-}, \zeta\right)=4+2 \ln (|1-2 \zeta|) \\
& +(2-\zeta) \ln \zeta+(2-\bar{\zeta}) \ln \bar{\zeta} \equiv-w_{1}(\zeta)
\end{aligned}
$$

Adding $w_{1}(\zeta) \Phi_{1,0}(x, \zeta)$ to $\Psi_{1,1}^{(0)}(x, \zeta)$, we obtain the correction function $\Psi_{1,1}(x, \zeta) \equiv$ $\chi(x, \zeta)$ that is continuous at the border points $x=\zeta$ and $x=\bar{\zeta}$ (see Fig. 28).

$$
\begin{aligned}
& \left.\Psi_{1,1}(x, \zeta)=\chi(x, \zeta) \equiv \int_{0}^{1} V(x, y)\left[\Phi_{1,0}(y, \zeta)-\Phi_{1,0}(x, \zeta)\right] d y-w(x, \zeta) \Phi_{0}(x, \zeta)\right] \\
& =\theta(0<x<\zeta<1 / 2)\left\{(1-4 \zeta) \frac{x}{2 \zeta}+\frac{1}{2 \zeta}[x \bar{x} \ln x+(1-x \bar{x}) \ln \bar{x}]\right. \\
& +\frac{2(x-\zeta)}{1-2 \zeta} \ln (\zeta-x)+\frac{\zeta-2 x \bar{\zeta}}{2 \zeta(1-2 \zeta)} \ln \zeta-\frac{x}{\zeta} \ln (1-2 \zeta) \\
& \left.+\frac{1-3 \zeta-2 x+2 x \zeta(2+\zeta)}{2 \zeta(1-2 \zeta)} \ln \bar{\zeta}-\frac{1-2 \zeta(x+3 \bar{\zeta})}{\zeta(1-2 \zeta)} \ln (\bar{\zeta}-x)\right\} \\
& -\{x \rightarrow \bar{x}\}+\theta(\zeta<x<\bar{\zeta})\left\{\frac { 1 - 2 x } { 2 ( 1 - 2 \zeta ) } \left[(1-4 \zeta)+\ln \bar{\zeta} \frac{\bar{\zeta}^{2}-2 \zeta}{\zeta}\right.\right. \\
& +(2-\zeta) \ln \zeta+2 \ln (1-2 \zeta)]+\frac{x-\zeta}{2 \zeta(1-2 \zeta)} \ln (x-\zeta) \\
& \left.-\frac{2-4 \zeta}{2 \zeta(1-2 \zeta)}(\bar{\zeta}-x)\right) \ln (\bar{\zeta}-x)+\frac{1-4 \zeta}{2 \zeta(1-2 \zeta)}[\bar{x} \ln \bar{x}-x \ln x] \\
& \left.+\frac{x^{2}+\bar{x}^{2}}{2(1-2 \zeta)} \ln \left(\frac{\bar{x}}{x}\right)\right\} \text {. }
\end{aligned}
$$

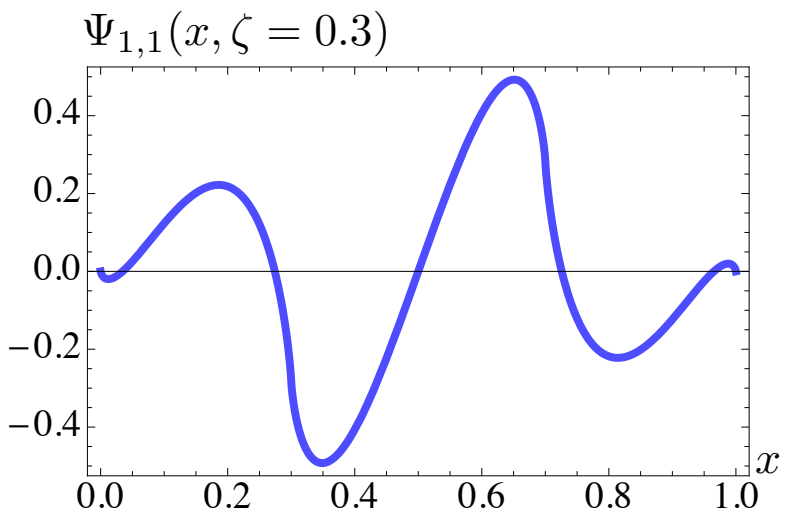

FIG. 28: Correction functions $\Psi_{1,1}^{(0)}(x, \zeta=0.3)$ with modified Ansatz.

This corresponds to the following $\Phi$-part of the Ansatz (Eq. 95)

$$
\Phi_{1}(x, \zeta, t)=e^{t v(x)}\left(\frac{|1-x / \zeta||1-x / \bar{\zeta}|}{|1-2 \zeta|}\right)^{2 t} \zeta^{t \zeta} \bar{\zeta}^{t \bar{\zeta}} \Phi_{1,0}(x, \zeta),
$$


for the function $\varphi_{1}(x, \zeta, t)$. The function $\Phi_{1}(x, \zeta, t)$ is illustrated in Fig. 29.

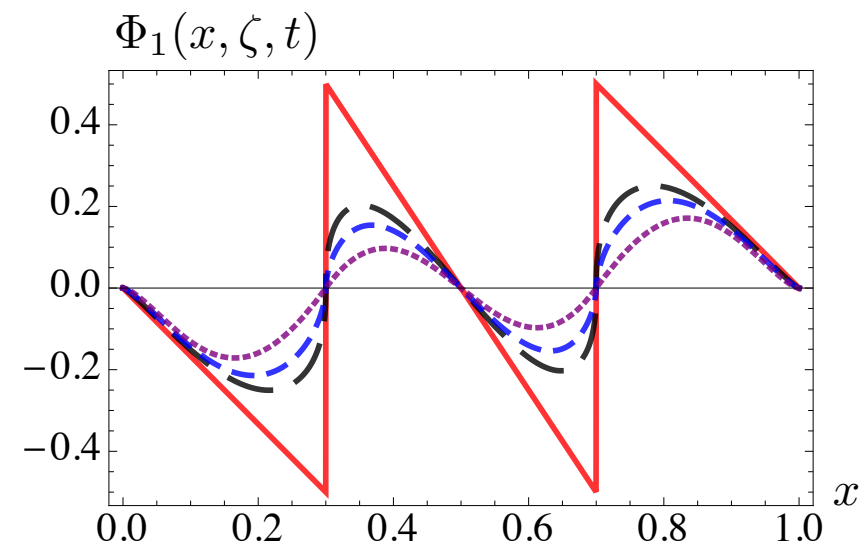

FIG. 29: Ansatz function $\Phi_{1}(x, \zeta=0.3, t)$ for $t=0,0.2,0.3,0.5$.

According to the Ansatz given in Eq. (95), after fixing the function $w(x)$ from the requirement of continuity of $\chi(x, \zeta)$, one should deal with the evolution equation Eq. (97) for the $\Psi$-part of the Ansatz. This equation specifies that $\partial \Psi_{1}(x, \zeta, t) / \partial t$ for $t=0$ is given by $\chi(x, \zeta)$. Thus, for small $t$, we can approximate $\Psi_{1}(x, \zeta, t)$ by $t \chi(x, \zeta)$. As one can see from Fig. 30, left, the correction due to the $\Psi$ term is rather small for $t=0.2$. It just reduces somewhat the amplitude of oscillations.
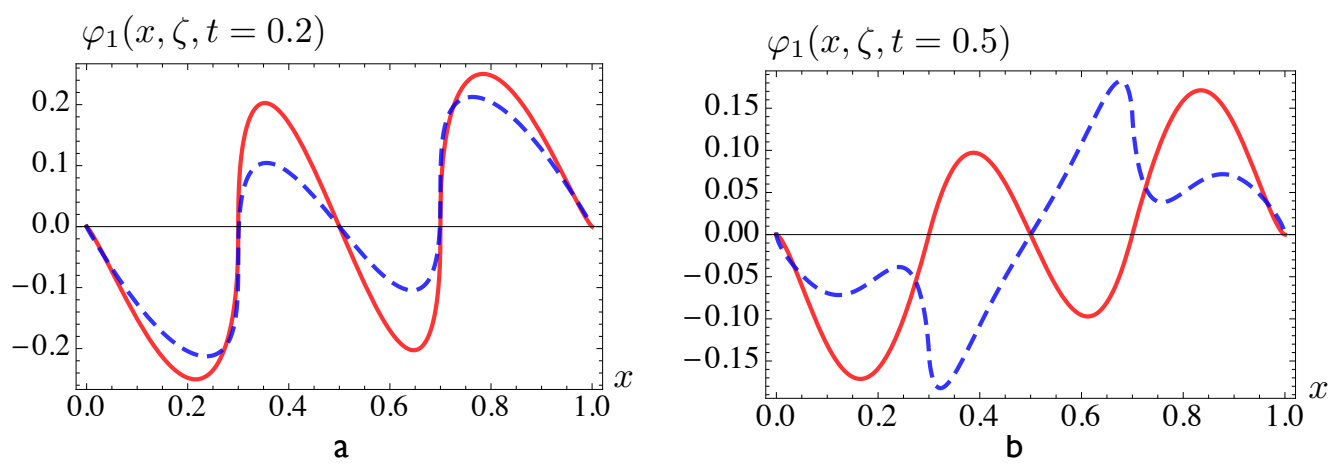

FIG. 30: Effect of inclusion of $\Psi_{1,1}(x, \zeta)$ correction for $t=0.2$ (a) and $t=0.4$ (b); $\zeta=0.3$ in both cases. Red line corresponds to $\varphi_{1}(x, \zeta, t)$ without correction and blue corresponds to $\varphi_{1}(x, \zeta, t)$ with correction.

However, the correction becomes more and more visible with growing $t$, see Fig. $30 \mathrm{~b}$, where the evolved function is shown for $t=0.4$ with and without the first $\Psi$-type correction term included. The total function is now clearly nonzero at the "border" points $x=\zeta$ and $x=1-\zeta$. This is because $\chi(x, \zeta)$ is nonzero at these 
points. As we discussed, the $\Psi$-part becomes dominant for large $t$ and brings the shape of $\varphi_{1}(x, \zeta, t)$ to the asymptotic form $x \bar{x}(2 x-1)$ of the antisymmetric DAs. We can see that, for $t=0.4$ already, the total function resembles the asymptotic shape $x \bar{x}(2 x-1)$. However, for such large $t$ values the simplest linear- $t$ approximation for $\Psi(x, \zeta, t)$ is too crude, and one should go beyond the first iteration.

As argued in the discussion after Eq. (95), it makes sense to split $\Psi(x, \zeta, t)$ into a part generated by iterations of $t \chi(x, \zeta)$, and the remainder $\delta \Psi(x, \zeta, t)$ given by iterations of the terms reflecting the deviation

$$
\delta \Phi(x, \zeta ; t) \equiv \Phi(x, \zeta ; t)-\Phi_{0}(x, \zeta)
$$

of the Ansatz function $\Phi(x, \zeta ; t)$ from its $t=0$ form $\Phi_{0}(x, \zeta)$. The starting term $\delta \Psi_{1}(x, \zeta, t)$ has a sharp behavior at the jump points of $\Phi_{0}(x, \zeta)$, acquiring an infinite slope there as $t \rightarrow 0$ (see Fig. 31a). The next iteration $\delta \Psi_{2}(x, \zeta, t)$ is shown in Fig. 31 b.

The decomposition explained for

$$
\Psi(x, \zeta, t) \approx t \chi(x, \zeta)+\delta \Psi(x, t)
$$

is informative in this case because of the infinite derivative parts of $\Psi(x, \zeta, t)$. As illustrated in Fig. 31, even though $\delta \Psi_{1}(x, \zeta=0.2, t=0.2)$ has almost infinite derivative parts at the borders $x=\zeta$ and $x=\bar{\zeta}$, it is still continuous. Up to this point of the calculation, all iterations are calculated analytically, however $\delta \Psi(x \zeta, t)$ is calculated numerically. As indicated in Fig. 31 and Fig 32 , contribution from $\delta \Psi_{1}$ and $\delta \Psi_{2}$ is very small and can be neglected.
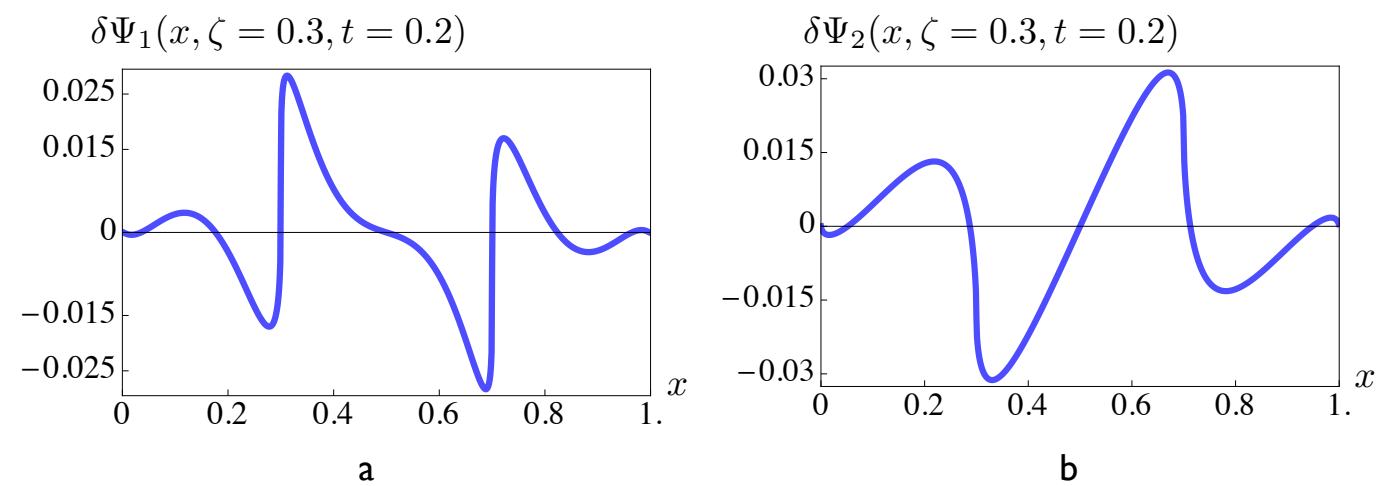

FIG. 31: (a) $\delta \Psi_{1}(x, \zeta, t)$, (b) $\delta \Psi_{1}(x, \zeta, t)(\zeta=0.2$ and $t=0.2)$. 
The amplitudes of both $\delta \Psi_{1}$ and $\delta \Psi_{2}$ are very small compared to the amplitude of $\Phi(x, \zeta, t)$. As suggested in the previous section $\Psi(x, \zeta, t) \approx t \chi(x, \zeta)$ is a good approximation. In Fig. 32, $\Phi(x, \zeta, t)$ with $\delta \Psi_{1}(x, \zeta, t)$ and $\delta \Psi_{2}(x, \zeta)$ is depicted. Contribution from $\delta \Psi(x, \zeta, t)$ is negligible.
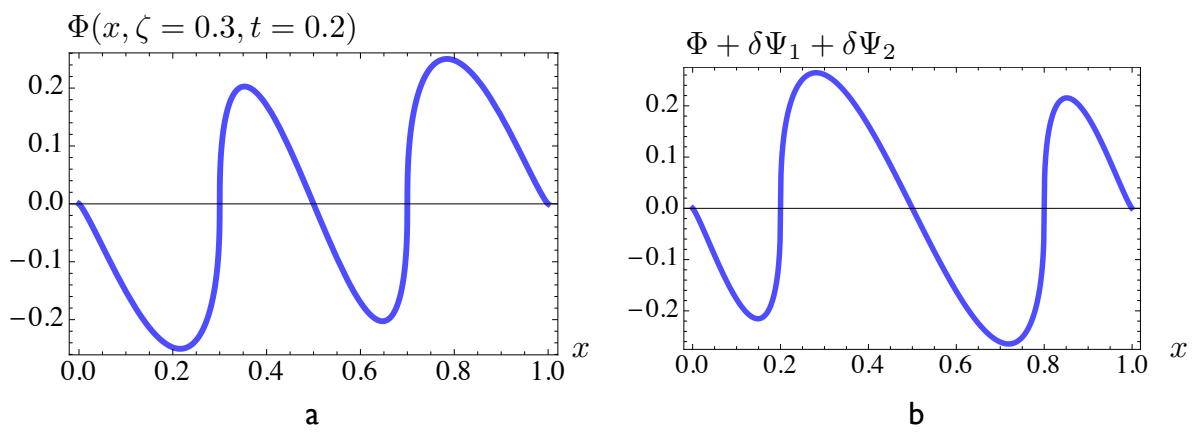

FIG. 32: (a) $\Phi(x, \zeta, t),(\mathrm{b}) \Phi(x, \zeta, t)$ and $\delta \Psi_{1}(x, \zeta, t)+\delta \Psi_{2}(x, \zeta, t) . \quad(\zeta=0.2$ and $t=0.2)$

In this chapter, we represented the application of our method to the jump part of the two-photon generalized amplitude. Next chapter, we demonstrate the evolution of the cusp part of the two-photon GDA. 


\section{CHAPTER 7}

\section{EVOLUTION OF THE CUSP PART OF THE TWO-PHOTON GDA}

\subsection{DECOMPOSITION}

In this section, we study the evolution of the second function, namely $\varphi_{2}(x, \zeta)$. Its initial form $\Phi_{2,0}(x, \zeta)$ is continuous for $x=\zeta$ and $x=\bar{\zeta}$ and is given by

$$
\begin{aligned}
\Phi_{2,0}(x, \zeta)= & -\left(\frac{x}{\zeta}\right) \frac{1+\zeta-4 \zeta^{2}+4(\zeta-x)}{2(1-\zeta)} \theta(x<\zeta)-\{x \rightarrow 1-x\} \\
& -\left(\frac{1-2 x}{1-2 \zeta}\right) \frac{1+\zeta-4 \zeta^{2}}{2(1-\zeta)} \theta(\zeta<x<\bar{\zeta}),
\end{aligned}
$$

as shown in Fig. 33 .

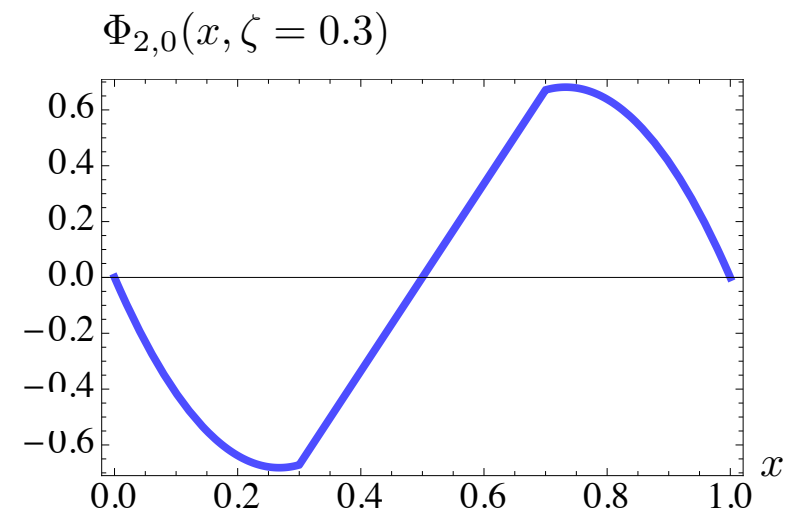

FIG. 33: Initial function $\Phi_{2,0}(x, \zeta)$ for $\zeta=0.2$.

We can separate this function

$$
\Phi_{2,0}(x, \zeta)=-\frac{1+\zeta-4 \zeta^{2}}{2(1-\zeta)} \Phi_{2,0}^{\mathrm{L}}(x, \zeta)+\Phi_{2,0}^{\mathrm{C}}(x, \zeta)
$$

into a term proportional to a linearized function

$$
\Phi_{2,0}^{\mathrm{L}}(x, \zeta)=\frac{x}{\zeta} \theta(x<\zeta)-\{x \rightarrow 1-x\}-\left(\frac{1-2 x}{1-2 \zeta}\right) \theta(\zeta<x<\bar{\zeta})
$$


and the remaining curvy part

$$
\Phi_{2,0}^{\mathrm{C}}(x, \zeta)=\frac{2 x(x-\zeta)}{\zeta \bar{\zeta}} \theta(x<\zeta)-\{x \rightarrow 1-x\}
$$

\subsection{EVOLUTION OF THE LINEARIZED PART}

Since $\Phi_{2,0}(x, \zeta)$ is a continuous function vanishing at the end points, the easiest way to get its evolution is to use a straightforward $t^{n}$ expansion with coefficients given by successive iterations of the evolution kernel with $\Phi_{2,0}(x, \zeta)$. The first iteration of $\Phi_{2,0}^{\mathrm{L}}(x, \zeta)$ gives

$$
\begin{aligned}
\Phi_{2,1}^{\mathrm{L}}(x, \zeta)= & -\frac{\theta(0<x<\zeta)}{\zeta(1-2 \zeta)}\{+\bar{x}[\bar{\zeta} \ln \bar{\zeta}-\zeta \ln \zeta] \\
& +(1-2 \zeta)[x+x \bar{x} \ln x+(1-x \bar{x}) \ln \bar{x}] \\
& +(\zeta-x) \ln (\zeta-x)-(\bar{\zeta}-x) \ln (\bar{\zeta}-x)\}-\{x \rightarrow \bar{x}\} \\
& -\frac{\theta(\zeta<x<\bar{\zeta})}{1-2 \zeta}\left\{(1-2 x)\left[1+\frac{\bar{\zeta}}{\ln } \bar{\zeta}\right]\right. \\
& \left.+2 x \bar{x} \ln \frac{\bar{x}}{x}+\left(\frac{x}{\zeta}-1\right) \ln \left(1-\frac{\zeta}{x}\right)-\left(\frac{\bar{x}}{\zeta}-1\right) \ln \left(1-\frac{\zeta}{\bar{x}}\right)\right\} \\
& +v(x) \Phi_{2,0}^{\mathrm{L}}(x, \zeta) .
\end{aligned}
$$

Here, as usual, $v(x)$ is $3 / 2+x \ln \bar{x}+\bar{x} \ln x$. The structure of the result is very similar to that of $\Phi_{2,1}$. However, the potentially singular logarithmic terms $\ln |x-\zeta|$ and $\ln |x-\bar{\zeta}|$ are accompanied in this case by $(x-\zeta)$ or $(x-\bar{\zeta})$ factors, respectively, and vanish at these points, though having singular derivatives there. Thus, the function $\Phi_{2,1}(x, \zeta=0.2)$, shown in Fig. 34 , is continuous at points $x=\zeta$ and $x=\bar{\zeta}$.

\subsection{EVOLUTION OF CURVY PART}

Initially, the support region for the curvy part $\Phi_{2,0}^{\mathrm{C}}(x, \zeta)$ is restricted by two segments $0<x<\zeta$ and $\bar{\zeta}<x<1$, as shown in Fig. 33 . 


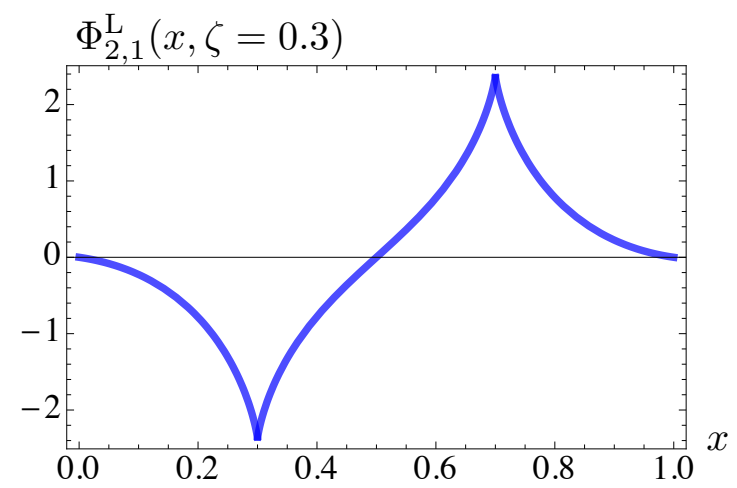

FIG. 34: First iteration function for linear part $\Phi_{2,1}^{L}(x, \zeta=0.2)$.

Its first iteration is given by

$$
\begin{aligned}
\Phi_{2,1}^{\mathrm{C}}= & -\frac{\theta(0<x<\zeta)}{\zeta \bar{\zeta}}\left\{x\left[3 x-4 \zeta+2 \zeta^{2}\right]+2[x \bar{x}(3+x-\zeta)+\bar{\zeta}] \ln \bar{x}\right. \\
& -2 \bar{x} \bar{\zeta} \ln \bar{\zeta}+2 x \bar{x}(x-\zeta) \ln x \\
& +2 x(\zeta-x) \ln (\zeta-x)-2 \bar{x}(\bar{\zeta}-x) \ln (\bar{\zeta}-x)\}-\{x \rightarrow \bar{x}\} \\
& -\frac{\theta(\zeta<x<\bar{\zeta})}{\zeta \bar{\zeta}}\left\{-(1-2 x) \zeta^{2}-2 x(x-\zeta) \ln x\right. \\
& +2 \bar{x}(\bar{\zeta}-x) \ln \bar{x}+2(1-2 x) \bar{\zeta} \ln \bar{\zeta} \\
& +2 x(x-\zeta) \ln (x-\zeta)-2 \bar{x}(\bar{\zeta}-x) \ln (\bar{\zeta}-x)\}+v(x) \Phi_{2,0}^{\mathrm{L}}(x, \zeta) .
\end{aligned}
$$
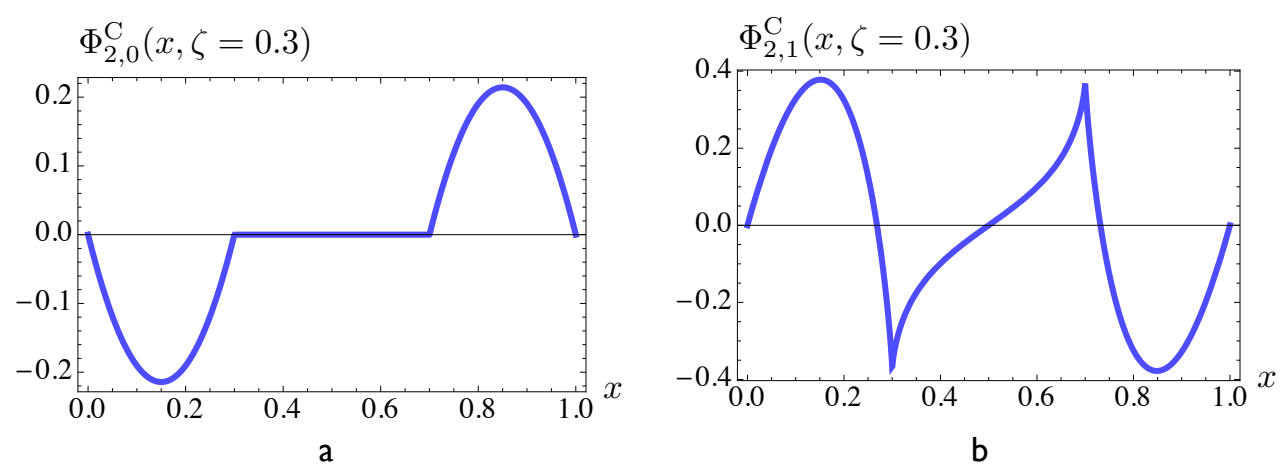

FIG. 35: Left: Initilal curvy function $\Phi_{2,0}^{\mathrm{C}}(x, \zeta)$ for $\zeta=0.2$. Right: First iteration function for curvy part $\Phi_{2,1}^{\mathrm{C}}(x, \zeta)$ for $\zeta=0.2$. 
One can see from Fig. 35 that evolution spreads the function into the $\zeta<x<\bar{\zeta}$ interval. Combining the results for the linearized and curvy parts, we arrive at the evolution pattern generated for $\varphi_{2}(x, \zeta ; t)$ by the first iteration (see Fig. 36).

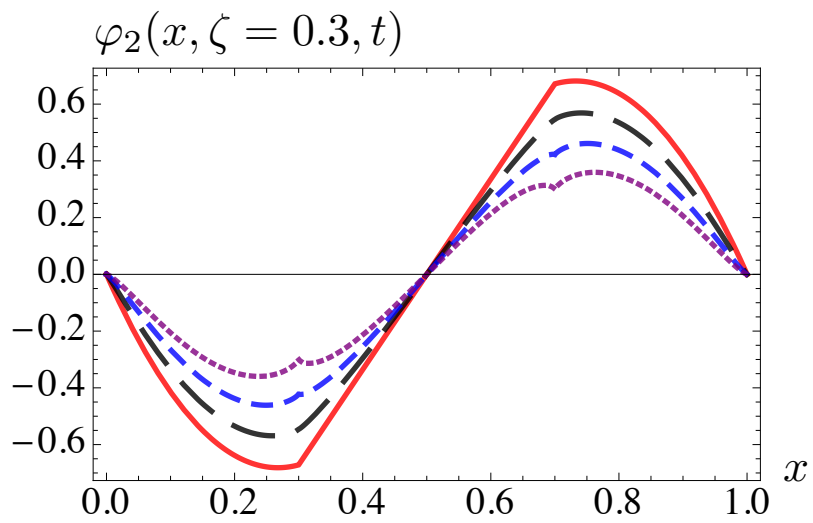

FIG. 36: Evolution of $\phi_{2}(x, \zeta, t)$ at $t=0$ (red), $t=0.2$ (black), $t=0.3$ (blue) and $t=0.5$ (purple).

\subsection{TOTAL RESULT}

Adding the result for $\Phi_{1}(x, \zeta, t)$ obtained in previous sections, we end up with the evolution of the total function $\Phi(x, \zeta, t)$ illustrated in Fig. 38.

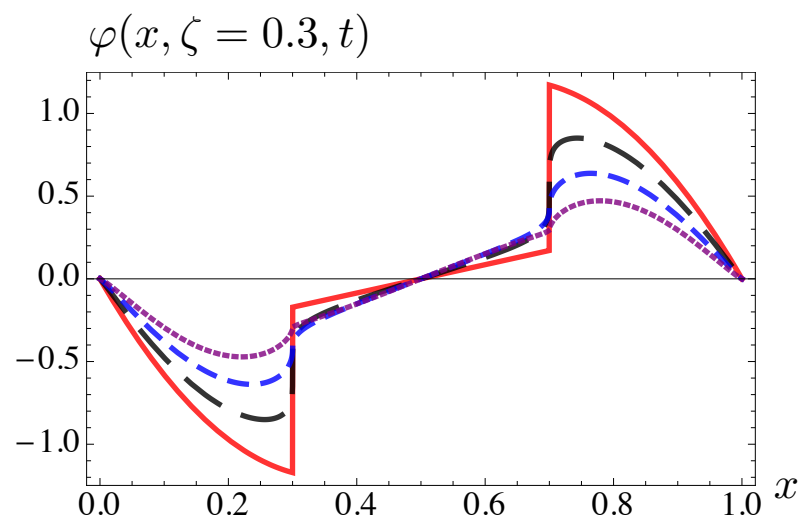

FIG. 37: Evolution of total GDA $\Phi(x, \zeta, t)$ at $t=0$ (red), $t=0.1$ (black), $t=0.2$ (blue) and $t=0.3$ (purple).

We can now compare our results with the results from Beiyad et.al. [38]. They 
have calculated the evolution of the two-photon GDA with Gegenbauer expansion, including 41 terms in Gegenbauer expansion in the intervals $0<x<\zeta$ and $\bar{\zeta}<x<1$. However, 201 terms were included in the region $\zeta<x<\bar{\zeta}$. They have reported that there is instability in the vicinity of $x=\zeta$ and $x=\bar{\zeta}$ because of the nature of the Gegenbauer expansions. We have emphasized the necessity of a new method other than the Gegenbauer expansion for evolution of singular DAs in the previous chapters. As one can see, our approach results in a smooter curve with only one or two iterations.

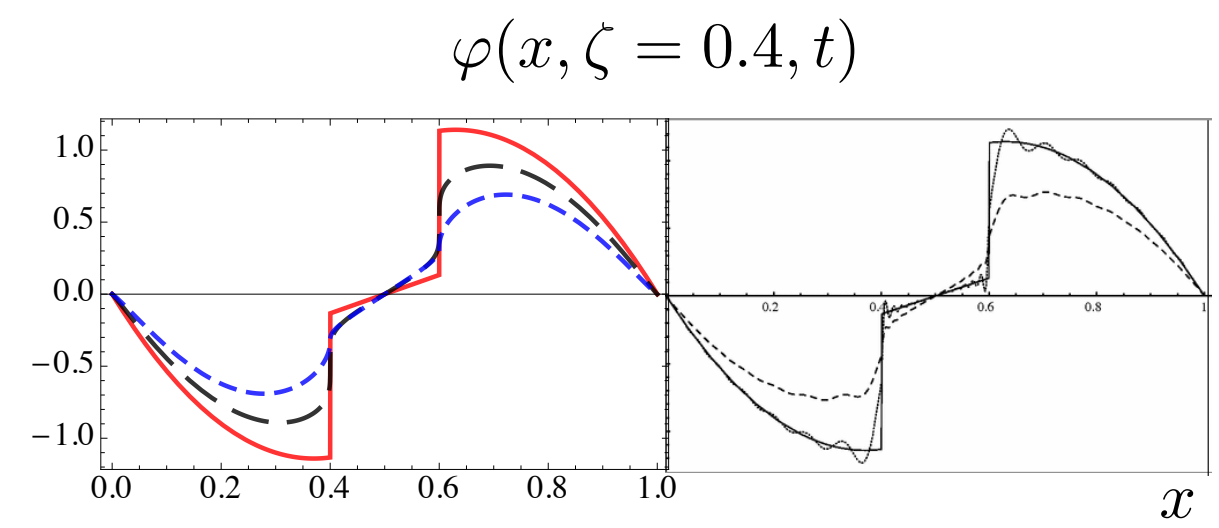

FIG. 38: Comparison between analytic result presented here and the numerical result from Ref. 38] at $\zeta=0.4$ and $t=0$ (red), $t=0.1$ (black), $t=0.2$ (blue). 


\section{CHAPTER 8}

\section{EVOLUTION IN DGLAP REGION}

The parton model completely ignores the dynamical role of gluons by neglecting the gluons radiated by quarks. As shown in Fig. 39, a gluon can be emitted by the quark before or after the virtual photon interacts with it $(a$ and $b)$. Another possibility in the order of $\alpha \alpha_{s}$ is that a gluon constituent in the hadron can contribute to DIS via pair production, i.e. $\gamma^{\star} g \rightarrow q \bar{q}(c$ and $d)$.

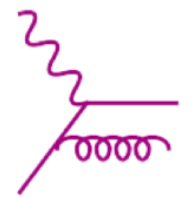

a

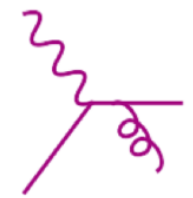

b

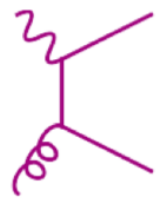

c

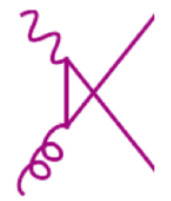

d

FIG. 39: $O\left(\alpha \alpha_{s}\right)$ contributions to $e p \rightarrow e X$.

One of the experimentally observable consequences of these diagrams may be illustrated by considering a frame, in which the proton and the virtual photon are moving towards each other, say the proton in the $+x$ direction and the virtual photon in the $-x$ direction. In this frame, the struck quark moves along the same axis as the incoming photon. In the parton model, final hadron states are produced in the direction of the virtual photon, with a spread of transverse momentum $p_{T}$ about $300 \mathrm{MeV}$, or inverse hadron size, according to the uncertainty principle. However, if gluons are emitted in DIS, then the jets produced by gluons and the struck quark have $p_{T}$ relative to the direction of the virtual photon.

The $e p$ cross-section can be written in terms of the $e q$ cross-section

$$
\begin{aligned}
\frac{\sigma_{T}^{e p}}{\sigma_{0}}\left(x, Q^{2}\right) & =\sum_{q} \int_{0}^{1} d z \int_{0}^{1} d y f_{q}(y) \delta(x-z y) \frac{\sigma_{T}^{e q}\left(z, Q^{2}\right)}{\sigma_{0}} \\
& =\sum_{q} \int_{x}^{1} \frac{d y}{y} f_{q}(y) \frac{\sigma_{T}^{e q}\left(z, Q^{2}\right)}{\sigma_{0}} .
\end{aligned}
$$


The total cross section of an eq collision for the diagrams $a$ ) and $b$ ) in Fig. 39 contains logarithmic dependence on $Q^{2}$

$$
\sigma^{e q}\left(\gamma^{\star} q \rightarrow q g\right)=e_{q}^{2} \sigma_{0}\left(\frac{\alpha_{s}}{2 \pi} P_{q q}(x) \log \frac{Q^{2}}{m^{2}}\right)
$$

where $P_{q q}(x)$ is the LO quark-quark DGLAP kernel and given by

$$
P_{q q}(x)=\left(\frac{1+x^{2}}{1-x}\right)_{+} .
$$

LO quark-quark DGLAP kernel reflects the fact that a quark with momentum fraction $x$ could have come from a parent quark with a larger momentum fraction $y$. The usual way to write the evolution equation in the DGLAP region is

$$
\frac{d f(x, t)}{d t}=\int_{x}^{1} \frac{d y}{y} P(y) f\left(\frac{x}{y}\right) .
$$

This equation might also be written as

$$
\frac{d f(x, t)}{d t}=\int_{x}^{1} \frac{d y}{y} P\left(\frac{x}{y}\right) f(y) .
$$

There are quite a few ways to solve the DGLAP evolution equations: Mellin transformation [39, 40], Laguerre mehod [41, 42] and "brute-force" iterations [43] are some of the ways to solve the DGLAP equation. There are also various other analytic calculations in the literature [44,47]. Numerical calculations [48, 49] are also widely used. The aim of this chapter is to demonstrate the application of our analytic method to the LO DGLAP evolution equation.

For our method, we use the notation $P(x, y)$ instead of the splitting function as a function of the ratio of $x$ to $y$, namely $P(x / y)$ in Eq. (127) and rewrite the evolution equation as

$$
\frac{d f(x, t)}{d t}=\int_{x}^{1} \frac{d y}{y} P(x, y) f(y) .
$$

The "+"-prescription is also encountered in the DGLAP case which regulates the singularities. Evolution equation can be arranged in such a way that "+"-prescription acts on the second variable, $y$ in this case, as we have done with the ERBL case explained in Chapter 2.

$$
\frac{d f(x, t)}{d t}=\int_{0}^{1} \frac{d y}{y} P(x, y)[f(y)-f(x)]+f(x) \int_{0}^{1}\left[\frac{P(x, z)}{z}-\frac{P(z, x)}{x}\right] d z .
$$

The first term of the expression becomes visibly regular as $f(x)-f(y)$ cancels the singularity of the kernel. The second integral is also regular. In the next section, we present an application of our method in the DGLAP region. 


\subsection{EVOLUTION OF $(1-x)^{3}$ PARTON DISTRIBUTION FUNCTION}

Here we study a regular function $(1-x)^{3}$. The reason for choosing a regular function is that singular PDFs are not encountered in actual models of PDFs. First, we present our results with the singular part of the kernel and later we represent the result with the full kernel as it was done for the ERBL case.

\section{Singular Part}

The singular part of the kernel is given by

$$
P_{\mathrm{s}}(x, y)=\frac{2 x / y}{1-x / y} \theta(x / y<1) .
$$

The second integral in Eq. 129

$$
s(x)=\left(\int_{0}^{1}\left[\frac{P(x, z)}{z}-\frac{P(z, x)}{x}\right] d z\right)
$$

is going to be the same for all the iterations, for that reason it can be taken out as an overall factor. The function $s(x)$ is given by

$$
s(x)=\int_{0}^{1}\left[\frac{P_{\mathrm{s}}((x, z)}{z}-\frac{P_{\mathrm{s}}((z, x)}{x}\right] d z=2+2 \ln \bar{x} .
$$

Therefore, one can propose the ansatz as

$$
f(x, t)=e^{t s(x)} F(x, t) .
$$

where $F(x, t)$ can be written as a Taylor series in $t$,

$$
F(x, t)=\sum_{n=0}^{\infty} \frac{t^{n} \rho_{n}(x)}{n !} .
$$

The recurrence relation for the expansion components $\rho_{n}$ is given by

$$
\rho_{n+1}(x)=\int_{0}^{1} \frac{d y}{y} P(x, y)\left[\sum_{\ell=0}^{n} \frac{n !}{(n-\ell) ! \ell !} \rho_{\ell}(y) L_{y x}^{n-\ell}-\rho_{n}(x)\right] .
$$

the explicit expression for the first expansion component $\rho_{1}(x)$ is given by

$$
\begin{aligned}
\rho_{1}(x) & =\int_{x}^{1} \frac{d y}{y} P(x, y)\left[\rho_{0}(y)-\rho_{0}(x)\right] \\
& =x\left[\bar{x}(5-3 x)+2\left(3-3 x+x^{2}\right) \ln x\right] .
\end{aligned}
$$



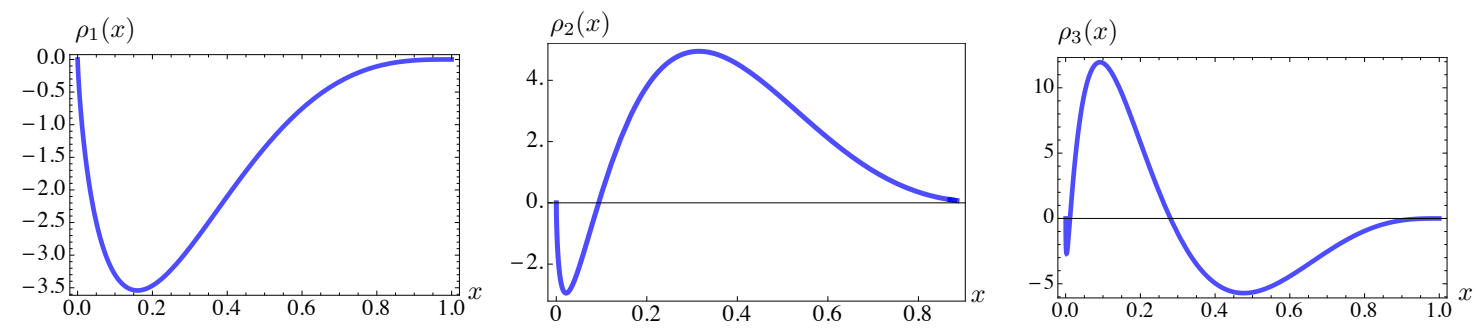

FIG. 40: Expansion components $\rho_{1}^{\text {sing }}(x)$ and $\rho_{2}^{\text {sing }}(x)$

Expressions of expansion components $\rho_{2}(x)$ and $\rho_{3}(x)$ are only graphically presented because of the lengthiness of the expressions. The graphical results for the expansion components are represented, in Fig. 40. The parton distribution function $f(x, t)$ in terms of expansion components is given by

$$
f(x, t)=e^{2 t} \bar{x}^{2 t}\left(\rho_{0}(x)+t \rho_{1}(x)+\frac{t^{2}}{2 !} \rho_{2}(x)+\frac{t^{3}}{3 !} \rho_{3}(x)\right)
$$

Fig. 41 shows the normalization versus $t$, where $t$ runs from zero to 0.5. Similar to the ERBL case, the normalization approaches one as more expansion components are added, as expected.

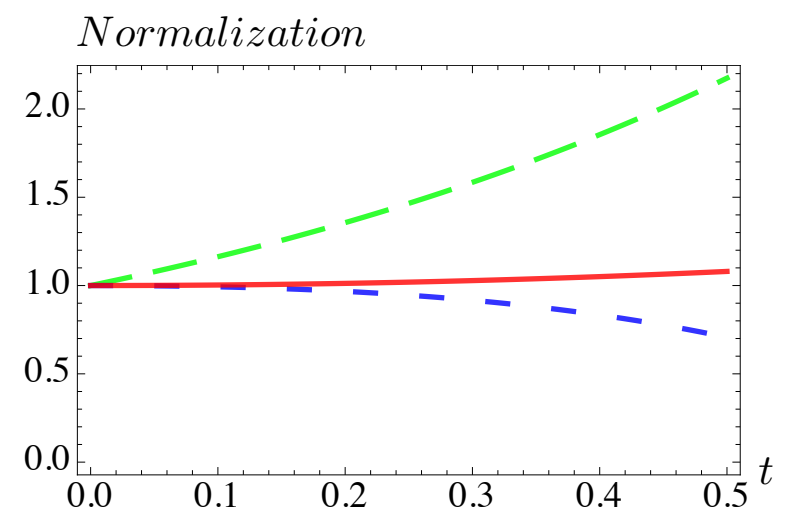

FIG. 41: Blue line is the normalization including $\rho_{0}(x)$ and $\rho_{1}(x)$, green line is the normalization including $\rho_{0}(x), \rho_{1}(x)$ and $\rho_{2}(x)$ and red line is the normalization including $\rho_{0}(x), \rho_{1}(x), \rho_{2}(x)$ and $\rho_{3}(x)$ which was calculated numerically

The evolution of the initial $(1-x)^{3}$ parton density is shown in Fig. 42 . 

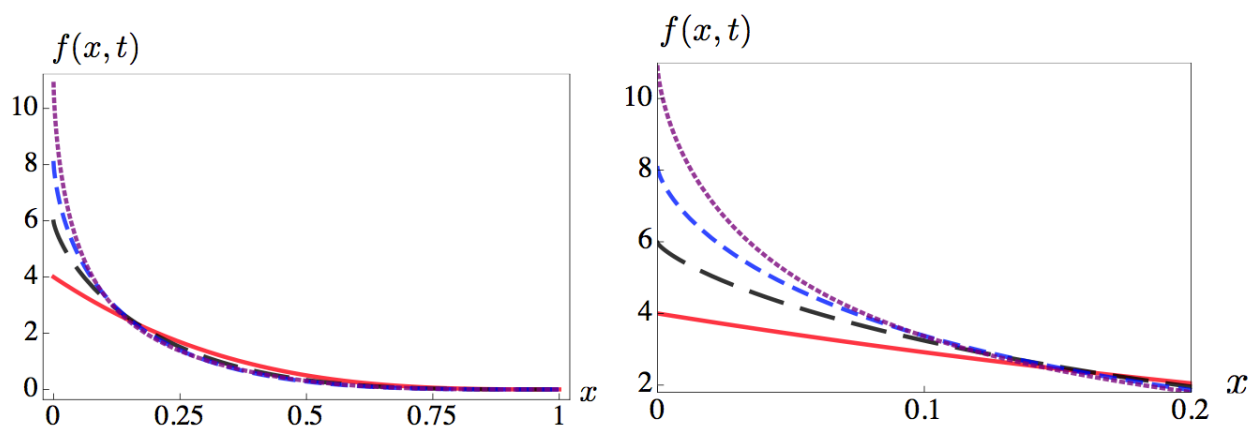

a

FIG. 42: (a) Starting from blue line: $t=0$ (red), $t=0.2$ (black), $t=0.4$ (blue), $t=0.5$ (purple) and (b) Closer look to $0<x<0.2$ region

\section{Adding the Non-Singular Part}

When the full DGLAP kernel is taken into account, $s(x)$ which is the second term in Eq. (129) becomes

$$
s(x)=\int_{0}^{1}\left[\frac{P((x, z)}{z}-\frac{P((z, x)}{x}\right] d z=\frac{1}{2}+x+2 \ln \bar{x}-\ln x
$$

Therefore, Ansatz for full DGLAP kernel might be modified as

$$
f(x, t)=e^{(x+1 / 2) t} \bar{x}^{2 t} x^{-t} F(x, t) .
$$

Evolution of $(1-x)^{3}$ parton distribution function is shown in Fig. 43 .

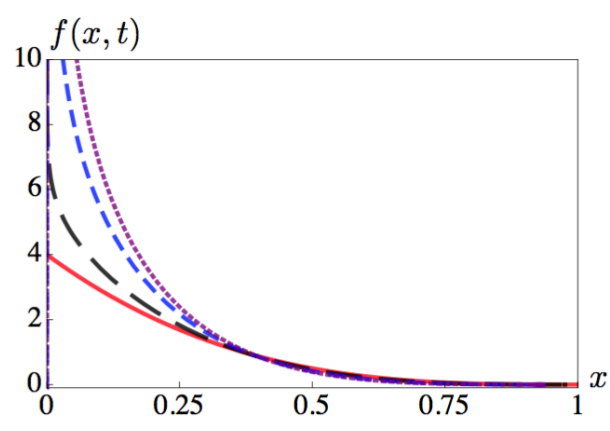

a

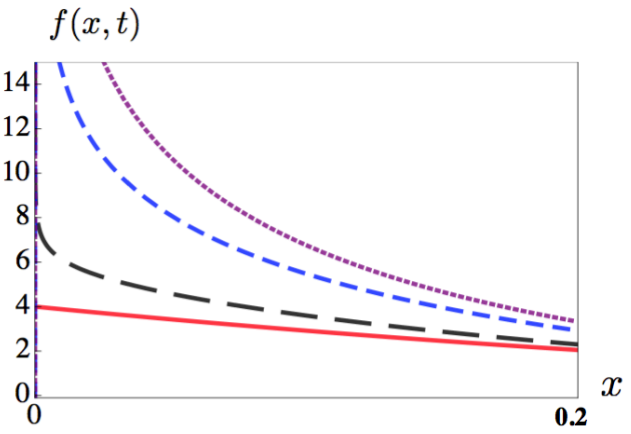

b

FIG. 43: Starting from blue line: $t=0$ (red), $t=0.2$ (black), $t=0.4$ (blue), $t=0.5$ (purple) and (b) Closer look to $0<x<0.2$ region 
The results are consistent with the expected results of of DGLAP evolution. Our method required only two iterations. Similar to the ERBL evolution, there is an upper limit on $t$, In other words, our method is effective up to $t \approx 0.5$. Further calculations can be performed with other methods mentioned in the beginning of this chapter, such as the Mellin transformation. 


\section{CHAPTER 9}

\section{EVOLUTION OF DOUBLE DISTRIBUTIONS}

Virtual Compton Scattering is a process that includes two photons, at least one of them is virtual. The use of deeply virtual Compton scattering (DVCS) was suggested by Ji [10] and Radyushkin [14] as a tool to extract new structure functions of the nucleon. DVCS provides a new ground to investigate the quark and the gluon structure of the nucleon and has led to the introduction of objects called Generalized Parton Distribution [10 15]. These objects are proposed to describe the soft part of the DVCS process. With today's knowledge, GPDs are the only tools provide complete information on the accessible structure of the nucleon. The limiting cases of GPDs are given by from factors, parton densities and distribution amplitudes.

Construction of theoretical models for GPDs is an inherent part of their studies. These models should satisfy several nontrivial requirements that follow from the most general principles of quantum field theory. In this context, one could mention polynomiality [15], positivity [50 52], hermiticity [16], time reversal invariance [15], etc. There are two ways to model GPDs. One way is a direct calculation in specific dynamical models such as the bag model, the chiral soliton model, light cone formalism, etc. Direct calculation has some difficulty in meeting these conditions. The second way is modeling with Double Distributions (DD) [13, 14, 16]. With this approach these complicated conditions are automatically satisfied by the relevant Feynman diagrams in perturbation theory. In particular, analysis of simple one-loop diagrams is the basis of the factorized DD Ansatz [52] (FDDA) that is a standard element of codes generating models for GPDs.

In fact, the commonly used version of the FDDA involves an assumption of universality of the DD profile function, which, though supported by one-loop examples, was not shown to be a mandatory property of double distributions. A possible way to go beyond the one-loop analysis, but still remain within the perturbation theory framework, is to incorporate pQCD evolution equations. Namely, the strategy is to take the expression for some one-loop diagram as the starting function for evolution, and use evolved patterns for modeling GPDs. 
Implementation of such a program faces some technical difficulties. In particular, a well known property of GPDs is that they are non-analytic at border points $x= \pm \xi$. For one-loop diagrams, this non-analyticity may take the form of cusps, jumps, and even delta-functions.

In this section, we will introduce some basic properties of DDs and we will present the evolution of an approximation to the delta function.

\subsection{DOUBLE DISTRIBUTION BASICS}

If the long-distance information is collected in the nonforward matrix element $\left.\langle p-r|\mathcal{O}(0, z)| p\rangle\right|_{z^{2}=0}$ of quark gluon light cone operators, one needs to generalize usual parton distributions while applying pQCD to deeply virtual Compton scattering (DVCS) $\gamma^{*}(q) N(p) \rightarrow \gamma\left(q^{\prime}\right) N\left(p^{\prime}\right)$ and hard exclusive electroproduction $\gamma^{*}(q) N(p) \rightarrow M\left(q^{\prime}\right) N\left(p^{\prime}\right)$ processes [10, 11, 13, 14, 52, 54]. These types of matrix elements can be parametrized by double distributions (DD's).

The kinematic variables of hard electroproduction processes are given by the initial momentum of the nucleon $p$ and momentum transferred $r=p-p^{\prime}$. In order to present the important features of the process, the kinematics can be set to $q^{2}=0$ (mmentum of the final photon or meson), $p^{2}=0, r^{2}=0$ and $p, q^{\prime}$ are light-cone 4 -vectors. The requirement $p^{2} \equiv(p+r)^{2}=p^{2}$ in this limit results in $p \cdot r=0$ which can be satisfied only if the two light-like momenta $p$ and $r$ are proportional to each other: $r=\zeta p$, where $\zeta$ is equal to the Bjorken variable $\zeta=x_{B j} \equiv Q^{2} / 2(p \cdot q)$.

Factorization of the hard electroproduction process $\gamma^{*}(q) N(p) \rightarrow M\left(q^{\prime}\right) N\left(p^{\prime}\right)$, accumulates all the nonperturbative information in the nonforward matrix element $\langle p-r|\psi(0) \psi(z)| p\rangle$. In the forward limit $r=0$, Fourier transformation of the matrix element provides the usual parton distributions. For quark operators, the double distributions are defined by the following representation [52]:

$$
\begin{aligned}
& \left\langle p^{\prime}, s^{\prime}\left|\bar{\psi}_{a}(0) \hat{z} E(0, z ; A) \psi_{a}(z)\right| p, s\right\rangle \mid z^{2}=0 \\
& =\bar{u}\left(p^{\prime}, s^{\prime}\right) \hat{z} u(p, s) \int_{0}^{1} \int_{0}^{1}\left(e^{-i x(p z)-i y(r z)} F_{a}(x, y ; t)-e^{i x(p z)-i \bar{y}(r z)} F_{\bar{a}}(x, y ; t)\right) \\
& \times \theta(x+y \leq 1) d x d y+\frac{1}{4 M} \bar{u}\left(p^{\prime}, s^{\prime}\right)(\hat{z} \hat{r}-\hat{r} \hat{z}) u(p, s) \\
& \int_{0}^{1} \int_{0}^{1}\left(e^{-i x(p z)-i y(r z)} K_{a}(x, y ; t)-e^{i x(p z)-i \bar{y}(r z)} K_{\bar{a}}(x, y ; t)\right) \theta(x+y \leq 1) d x d y
\end{aligned}
$$

Here the relation between the two components of the double distributions and 
the quark and antiquark parton densitiesare

$$
\int_{0}^{1-x} F_{a}(x, y ; t=0) d y=f_{a}(x) \quad ; \quad \int_{0}^{1-x} F_{\bar{a}}(x, y ; t=0) d y=f_{\bar{a}}(x),
$$

Parton picture of the double distribution is depicted in Fig. 44.

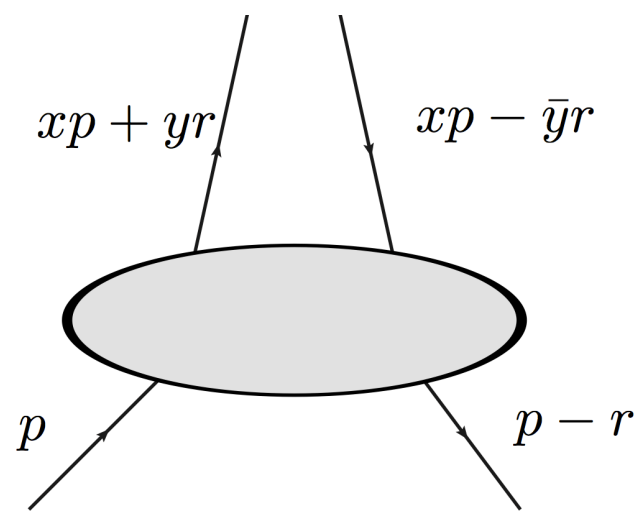

FIG. 44: Parton picture for double distributions

Double distributions $F(x, y ; t)$ are logaritmically divergent. This requires the dependence of the distribution $F(x, y ; t)$ on the renormalization scale $\mu$. This $\mu$ dependence $F(x, y ; t \mid \mu)$ is governed by the following evolution equation,

$$
\begin{aligned}
\mu \frac{d}{d \mu} F_{a}(x, y ; t \mid \mu)= & \int_{0}^{1} \int_{0}^{1} \sum_{b} d \xi d \eta \theta(\xi+\eta \leq 1) \\
& \times R^{a b}(x, y ; \xi, \eta) F_{b}(\xi, \eta ; t \mid \mu),
\end{aligned}
$$

where $a, b$ corresponds to quark or gluon.

The integration of $F(x, y ; t \mid \mu)$ over $y$ corresponds to parton distribution function $f_{a}(x \mid \mu)$ whose evolution is governed by DGLAP equations

$$
\mu \frac{d}{d \mu} f_{a}(x \mid \mu)=\int_{x}^{1} P_{a b}(x / \xi) f_{b}(\xi \mid \mu) \frac{d \xi}{\xi} .
$$

This relation suggests that the kernel $R^{a b}(x, y ; \xi, \eta)$ should satisfy the following relation,

$$
\int_{0}^{1-x} R^{a b}(x, y ; \xi, \eta) d y=\frac{1}{\xi} P^{a b}(x / \xi)
$$


Moreover, integration of $F(x, y ; t \mid \mu)$ over $x$ corresponds to the distribution amplitude, which implies that the kernel $R^{Q Q}(x, y ; \xi, \eta)$ should satisfy also the following relation,

$$
\int_{0}^{1-y} R^{Q Q}(x, y ; \xi, \eta) d x=V^{Q Q}(y, \eta)
$$

For the gluon kernel the relation is

$$
\int_{0}^{1-y} \frac{x}{\xi} R^{G G}(x, y ; \xi, \eta ; g) d x=V^{G G}(y, \eta ; g) .
$$

In this thesis we are going to work only with the quark kernel whose explicit form is

$$
\begin{aligned}
R^{Q Q}(x, y ; \xi, \eta) & =\frac{\alpha_{s}}{\pi} C_{F} \frac{1}{\xi}\{\theta(0 \leq x / \xi \leq \min \{y / \eta, \bar{y} / \bar{\eta}\} \\
& +\frac{\theta(0 \leq x / \xi \leq 1) x / \xi}{(1-x / \xi)}\left[\frac{1}{\eta} \delta(x / \xi-y / \eta)+\frac{1}{\bar{\eta}} \delta(x / \xi-\bar{y} / \bar{\eta})\right] \\
& \left.-\delta(1-x / \xi) \delta(y-\eta)\left[\frac{1}{2}+2 \int_{0}^{1} \frac{z}{1-z} d z\right]\right\}
\end{aligned}
$$

The last term corresponds to the "+"-prescription explained in the previous sections. Kernel without the " + "-prescription part is singular at $1 /(x-\xi), 1 /(y-\eta)$ and $1 /(\bar{y}-\bar{\eta})$. The integral variable $z$ in the last term can be selected as $x / \xi, y / \eta$ or $\bar{y} / \bar{\eta}$ depending on the chosen singularity.

\subsubsection{SINGULAR PART}

Once again, it is instructive to study the singular part first. The singular part of the kernel is

$$
\begin{aligned}
R^{q q}(x, y ; \xi, \eta) & =\frac{\alpha_{s}}{\pi} C_{F} \frac{1}{\xi}\left\{\frac{\theta(0 \leq x / \xi \leq 1) x / \xi}{(1-x / \xi)}\left[\frac{1}{\eta} \delta(x / \xi-y / \eta)+\frac{1}{\bar{\eta}} \delta(x / \xi-\bar{y} / \bar{\eta})\right]\right. \\
& \left.-\delta(1-x / \xi) \delta(y-\eta)\left[2 \int_{0}^{1} \frac{z}{1-z} d z\right]\right\} .
\end{aligned}
$$

Lets concentrate on the first term,

$$
\left(\frac{1}{\xi}\left\{\frac{\theta(0 \leq x / \xi \leq 1) x / \xi}{(1-x / \xi)}\left[\frac{1}{\eta} \delta(x / \xi-y / \eta)\right]\right\}\right)
$$


and try to find the "+" term for this term. The delta function can be rearranged as $\xi \delta(x-y \xi / \eta)$

$$
\begin{gathered}
\int_{0}^{1-y} d x\left[\frac{\theta(0 \leq x / \xi \leq 1) x / \xi}{(1-x / \xi)}\left[\frac{1}{\eta} \delta(x / \xi-y / \eta)\right]\right]_{+}=0 \\
\int_{0}^{1-y} d x \frac{\theta(0 \leq x / \xi \leq 1) x / \xi}{\eta(1-x / \xi)} \delta(x-y \xi / \eta) F(\xi, \eta) \\
=\frac{\theta(0 \leq y / \eta \leq 1) y / \eta}{\eta(1-y / \eta)} F(\xi, \eta)
\end{gathered}
$$

It means that for plus-type distribution the subtraction term for the first part of the singular kernel should be

$$
\frac{\theta(0 \leq y / \eta \leq 1) y / \eta}{\eta(1-y / \eta)} F(\xi, \eta)
$$

i.e., $z$ in Eq. 148 is $z=z^{\prime} / \eta$. Now we may study the evolution equation for only this term,

$$
\begin{aligned}
\frac{d F(x, y ; t)}{d t} & =\int_{0}^{1} \int_{0}^{1} \frac{\theta(0 \leq x / \xi \leq 1) x / \xi}{\xi \eta(1-x / \xi)} F(\xi, \eta ; t) \delta(x / \xi-y / \eta) \theta(\xi+\eta \leq 1) d \xi d \eta \\
& -\int_{0}^{1} \int_{0}^{1}[\delta(1-x / \xi) \delta(y-\eta) \theta(\xi+\eta \leq 1) \\
& \left.\times \frac{F(\xi, \eta ; t)}{\xi} d \xi d \eta \int_{0}^{\eta} \frac{z^{\prime} / \eta}{1-z^{\prime} / \eta} \frac{d z^{\prime}}{\eta}\right] .
\end{aligned}
$$

After some simple algebra, the following expression is achieved

$$
\begin{aligned}
\frac{d F(x, y ; t)}{d t} & =\int_{0}^{1} \frac{\theta(0 \leq y / \eta \leq 1) y / \eta}{y(1-y / \eta)} F\left(\frac{x \eta}{y}, \eta ; t\right) \theta(\eta(x / y+1) \leq 1) d \eta \\
& -F(x, y ; t) \theta(x+y \leq 1) \int_{0}^{y} \frac{z^{\prime}}{y-z^{\prime}} \frac{d z^{\prime}}{y} .
\end{aligned}
$$

In the first term of the expression above, we have two step functions which indicate the upper and lower limits of $\eta$ integration. The integration line is demonstrated in Fig. 45

At this point of the calculation, one can change $z^{\prime}$ to $\eta$ and add and subtract $y$ to the nominator of the second term.

$$
\begin{aligned}
\frac{\partial F(x, y ; t)}{\partial t} & =\int_{y}^{\frac{y}{y+x}} \frac{d \eta}{\eta-y} F\left(\frac{x \eta}{y}, \eta ; t\right)+F(x, y ; t) \theta(x+y \leq 1) \int_{0}^{y} \frac{d \eta}{\eta-y} \\
& +F(x, y ; t) \theta(x+y \leq 1)
\end{aligned}
$$



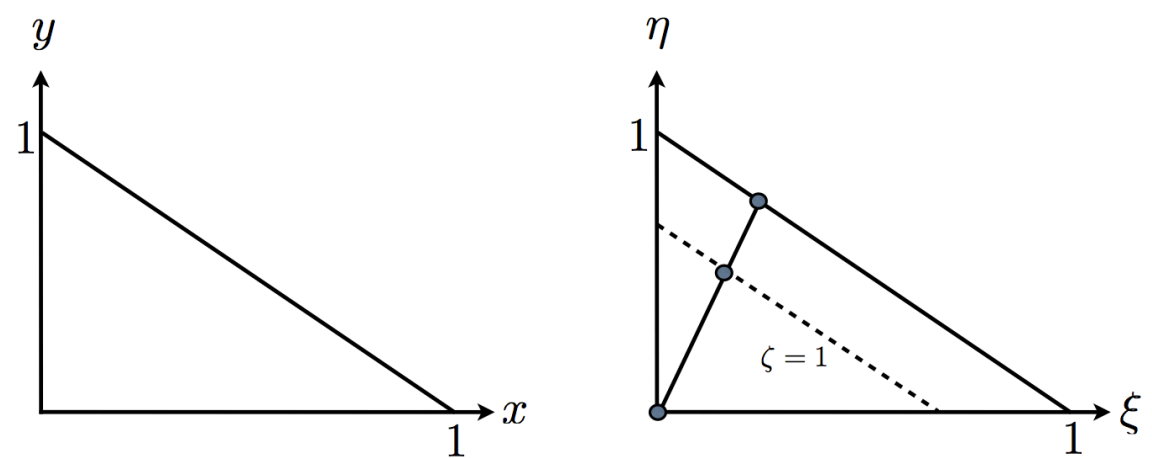

FIG. 45: The triangle on the left is the integral domain before the change of variable $\left(z^{\prime} \rightarrow\right.$ eta $)$ and the right triangle is the integral line and the limits after the change of variables. The integral is divergent when $x=\xi$ and $y=\eta$ (point on $\zeta=1$ line) which is regularized by the " + " prescription. The line from 0 to $\zeta=1$ represents the line of integration for the second part of the singular kernel.

The integral can be arranged as it was done in previous chapters, to modify the plus-prescription with respect to second variable,

$$
\frac{\partial \varphi(x, t)}{\partial t}=\int_{0}^{1} V(x, y)[\varphi(y, t)-\varphi(x, t)] d y-\varphi(x, t) \int_{0}^{1}[V(y, x)-V(x, y)] d y
$$

So we add and subtract $\int_{y}^{y /(x+y)} \frac{d \eta}{\eta-y} F(x, y ; t)$ which results in

$$
\begin{aligned}
\frac{\partial F(x, y ; t)}{\partial t} & =\int_{y}^{\frac{y}{y+x}} \frac{d \eta}{\eta-y}\left[F\left(\frac{x \eta}{y}, \eta ; t\right)-F(x, y ; t)\right] \\
& +\underbrace{F(x, y ; t) \int_{y}^{\frac{y}{x+y}} \frac{d \eta}{\eta-y}+F(x, y ; t) \int_{0}^{y} \frac{d \eta}{\eta-y}}_{F(x, y ; t) \int_{0}^{\frac{y}{x+y}} \frac{d \eta}{\eta-y}} \\
& +F(x, y ; t) \theta(x+y \leq 1) .
\end{aligned}
$$

Here, we have combined the two integrals under one integration, and the result of the integration is

$$
F(x, y ; t) \int_{y}^{\frac{y}{x+y}} \frac{d \eta}{\eta-y}=F(x, y ; t) \ln \left(\frac{x+y-1}{x+y}\right)
$$

For the second term in Eq. 148, we follow the same procedure. This time the term 
for the plus-type distribution is $\int_{\eta}^{1} \frac{\bar{z}^{\prime} / \bar{\eta}}{1-\bar{z}^{\prime} / \bar{\eta}}$. In this case, we have

$$
\begin{aligned}
\frac{\partial F(x, y ; t)}{\partial t} & =\int_{0}^{y} \frac{d \eta}{y-\eta}\left[F\left(\frac{x \bar{\eta}}{\bar{y}}, \eta ; t\right)-F(x, y ; t)\right] \\
& +\underbrace{F(x, y ; t) \int_{0}^{y} \frac{d \eta}{y-\eta}+F(x, y ; t) \int_{y}^{1} \frac{d \eta}{y-\eta}}_{F(x, y ; t) \int_{0}^{1} \frac{d \eta}{y-\eta}} \\
& +F(x, y ; t) \theta(x+y \leq 1),
\end{aligned}
$$

where the combined integral is equal to

$$
F(x, y ; t) \int_{0}^{1} \frac{d \eta}{y-\eta}=\ln \frac{y}{y-1} .
$$

So the final result, which does not include any divergent integrals is

$$
\begin{aligned}
\frac{\partial F(x, y ; t)}{\partial t} & =\int_{y}^{\frac{y}{y+x}} \frac{d \eta}{\eta-y}\left[F\left(\frac{x \eta}{y}, \eta ; t\right)-F(x, y ; t)\right] \\
& +\int_{0}^{y} \frac{d \eta}{y-\eta}\left[F\left(\frac{x \bar{\eta}}{\bar{y}}, \eta ; t\right)-F(x, y ; t)\right] \\
& +F(x, y ; t)\left[2+\ln \left(\frac{y(1-x-y)}{\bar{y}(x+y)}\right)\right) .
\end{aligned}
$$




\subsubsection{EVOLUTION OF $(y(1-x-y))^{10}$ DOUBLE DISTRIBUTION}

As an application of this rearranged evolution equation, we can study an approximation to the delta function. The importance of studying the evolution of the delta function is because of the appearance of delta functions in D-terms appearing in GPDs. As an illustration, we studied the function $F(x, y, t=0)=(y(1-x-y))^{a}$. In this work, we are using $a=10$. The first correction term is calculated analytically. The graphs below show the $x$-profiles and y-profiles of the $F(x, y, t)=(y(1-x-y))^{10}$ . The $y$-profiles are normalized in order to make the change in the shape of the function visible when the evolution is turned on. The figure below shows the $x$-profile and $y$-profile at different $x$ values of the function $F(x, y, t=0)(y(1-x-y))^{10}$. To

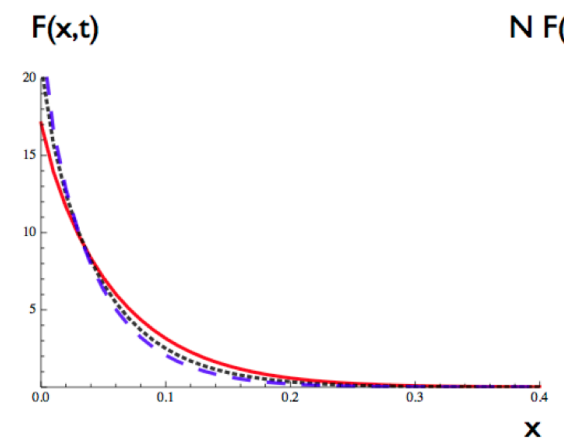

$N F(x,(I-x) y ; t=0.2)$

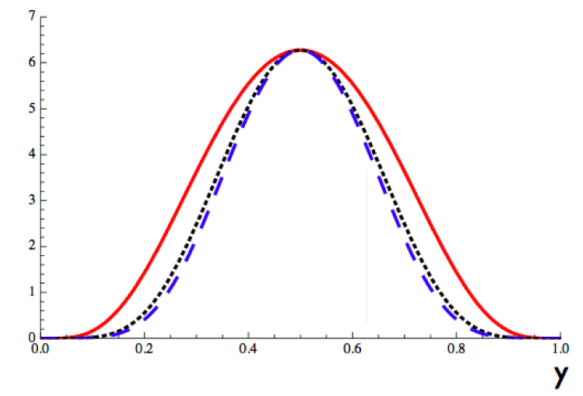

FIG. 46: $x$-profile at $t=0$ (red), $t=0.2$ (black) and $t=0.4$ (blue) and $y$ profiles at $x=0$ (red), $x=0.3$ (black) and $x=0.5$ (blue) for evolution of $(y(1-x-y))^{10}$.

summarize, in this chapter we gave and introduction to double distributions and their evolution. We presented an example for evolution of DDs by studying the evolution of $F(x, y, t=0)=(y(1-x-y))^{10}$. 


\section{CHAPTER 10}

\section{CONCLUSION AND FUTURE APPLICATIONS}

The introduction of the factorization theorem has broadened the spectrum of applications of perturbative QCD. Factorization provides the absorbtion of the nonperturbative (long-distance) part of an event into the objects which can then be measured experimentally, leaving the perturbative part (short-distance) to be calculated. The objects which contain the non-perturbative information are the distribution amplitudes and parton distribution functions. The evolution of distribution amplitudes and parton distribution functions provides information about these nonperturbative objects at different momentum transfer $Q^{2}$. In other words, if the DA (or PDF) is known at an energy level $Q^{2}$, one can evolve this function to different energies. The evolution of DAs and PDFs are governed by ERBL and DGLAP evolution equations.

The standard method of calculating the ERBL evolution is the Gegenbauer expansion. This method is a very effective way to calculate the evolution of regular DAs, i.e. DAs which are zero at end points $x=0$ and $x=1$ and continuous within that interval. In the case of a singular DA, the method of expansion in Gegenbauer polynomials requires an infinite number of terms in order to eliminate singularities in the initial distributions. We present a new approach, which is very efficient in application to functions that do not vanish at the end points or have jumps and cusps inside the support region $0<x<1$. While the Gegenbauer expansion produces logarithmically divergent terms at each iteration, in our method the logarithmic singularities are summed from the start, which immediately produces a continuous curve, with only one or two iterations needed afterwards in order to get precise results.

First, we applied our method to an initial DA that is constant in the whole $0 \leq x \leq 1$ interval. The evolution kernel is studied in two parts, singular and whole. The reason for this separation is to investigate the singularities corresponding to the singular part of the kernel. The evolution equation was arranged in such a way that the first term in the evolution equation has a structure of a "+"-prescription with respect to the integration variable. The second term in the arranged evolution equation, which is also finite can be absorbed in the exponential as an overall factor, 
i.e. $\varphi(x, t)=e^{t v(x)} \Phi(x, t)$. Then, $\Phi(x, t)$ can be written as a series in $t$. The leading term gives $x \bar{x}$ evolution with the change of the evolution parameter $t$. For the accompanying factor, two further terms in the $t^{N}$ expansion were found. We have compared our results with the ones from Gegenbauer expansion. Fifty terms of Gegenbauer expansion could still not provide the desired asymptotic form while only two iterations were sufficient with our method.

We also applied the method to an initial antisymmetric DA which is constant in each of its two parts $0 \leq x \leq 1 / 2$ and $1 / 2 \leq x \leq 1$. In this case, there is an extra factor $|1-2 x|^{2 t}$ that takes care of the jump in the middle at $x=1 / 2$. Two correction terms were also calculated. The results show good convergence for $t \leq 1 / 2$. Comparison of our method and the Gegenbauer expansion is presented. It should be noted that for $t \geq 1 / 2$, the evolved DA is rather close to the asymptotic form, and one can use the standard method of the Gegenbauer expansion which is well convergent for such functions.

Then we applied our method for studying the evolution of the (logarithmic $Q^{2}$ derivative of the) two-photon GDA. The initial DA of the two-photon GDA is zero at end points $x=0$ and $x=1$, butr it has jumps and cusps at $x=\zeta$ and $x=\bar{\zeta}$. We separated the initial DA into two parts, the jump part and the cusp part.

Jump part includes antisymmetric jumps at $x=\zeta$ and $x=\bar{\zeta}$. We introduced an Ansatz in the form of

$$
\varphi(x, \zeta, t)=\Phi(x, \zeta, t)+\Psi(x, \zeta, t)
$$

where $\Phi(x, \zeta, t)=e^{t[v(x)+w(x, \zeta)]} \Phi_{0}(x, \zeta)$ with $w(x)$ absorbing major features of the evolution of the starting distribution in the vicinity of the jump points. $\Psi(x, \zeta, t)$ is a regular function vanishing at $t=0$. The first expansion component of the jump part has logarithmic singularities as expected. These terms are absorbed in the Ansatz. However, resulting function of the first correction has still jumps after subtraction of singularities at $x=\zeta$ and $x=\bar{\zeta}$. To avoid these singularities, we adjusted the Ansatz in such a way to make $\partial \Psi(x, \zeta, t) / \partial t$ at $t=0(\chi(x, \zeta))$ a continuous function. For small $t$, the deviation of the Ansatz function $\Phi(x, \zeta, t)$ from its $t=0$ shape, called $\delta \Phi(x, \zeta, t)$, has a rather sharp behavior at $x=\zeta$ and $x=\bar{\zeta}$. This results in a rather sharp behavior of $\delta \Psi_{n}(x, \zeta, t)$, which is the correction function to $\Psi(x, \zeta, t)$. For this reason we split $\Psi(x, \zeta, t)$ into smooth part $\Psi_{\chi}(x, \zeta, t)$ and the remainder $\delta \Psi(x, \zeta, t)$. 
The final structure of our Ansatz is

$$
\varphi(x, \zeta, t)=\Phi(x, \zeta, t)+\delta \Psi(x, \zeta, t)+\Psi_{\chi}(x, \zeta, t)
$$

We have shown that the amplitudes of $\delta \Psi_{1}$ and $\delta \Psi_{2}$ are very small compared to the amplitude of $\Phi(x, \zeta, t)$. This result shows that $\Psi(x, \zeta, t) \approx t \chi(x, \zeta)$ is a good approximation and is calculable analytically.

In cusp part of the calculation, we split the function into a linearized and a curvy part. The linearized part is calculated with straightforward $t^{n}$ expansion. We presented the first iteration for the linearized part. The evolution of the curvy part showed that evolution spreads the support region of the function into the whole interval $0<x<1$. The initial support region for the curvy part is $0<x<\zeta$ and $\bar{\zeta}<x<1$, while after evolution turns on the function spreads into the $\zeta<x<\bar{\zeta}$ region.

We have also presented the application of our method to the DGLAP evolution equation and the evolution of double distributions. For the DGLAP case, we have shown the evolution of a simple non-singular PDF, $(1-x)^{3}$. Our results show consistency with the expected results up to $t \approx 0.5$. For the evolution of double distributions, we have shown results for evolution of function $[y(1-x-y)]^{10}$ as an approximation to a Delta function.

The results presented in this thesis were published in Refs. [55]57]. The methods developed may be extended to generalized parton distributions. In that case, two strategies are possible. The first strategy is to use a direct evolution equation for GPD. In that case, both the GPD and the evolution kernel depend on the skewness parameter $\zeta$, which is analogous to the parameter $\zeta$ encountered in the two-photon GDA studies. Another strategy is to use the evolution equation for the double distribution $F(\beta, \alpha ; t)$. In this case, no skewness parameter is present in the evolution equation, and dependence on $\xi$ appears after one performs the conversion of the double distribution into a GPD. In both cases, various aspects of our methods of analytic evolution may be used. In particular, GPDs are nonanalytic at the border points $x \pm \xi$, having there cusps, while model DDs may have a singular structure (jumps, delta functions) present in their initial shape. 


\section{BIBLIOGRAPHY}

[1] A. V. Efremov and A. V. Radyushkin, Phys. Lett. B 94, 245 (1980).

[2] G. P. Lepage and S. J. Brodsky, Phys. Rev. D 22, 2157 (1980).

[3] H. Fritzsch, M. Gell-Mann and H. Leutwyler, Phys. Lett. B 47, 365 (1973).

[4] M. Gell-Mann, Phys. Lett. 8, 214 (1964).

[5] G. Zweig, CERN-TH-401.

[6] G. Zweig, Developments in The Quark Theory of Hadrons, edited by D. Lichtenberg and S. Rosen Vol 2, pp. 22-101.

[7] V. N. Gribov and L. N. Lipatov, Sov. J. Nucl. Phys. 15, 438 (1972) [Yad. Fiz. 15, $781(1972)]$.

[8] G. Altarelli and G. Parisi, Nucl. Phys. B 126, 298 (1977).

[9] Y. L. Dokshitzer, Sov. Phys. JETP 46, 641 (1977) [Zh. Eksp. Teor. Fiz. 73, 1216 (1977)].

[10] X. -D. Ji, Phys. Rev. Lett. 78, 610 (1997).

[11] X. -D. Ji, Phys. Rev. D 55, 7114 (1997).

[12] A. V. Radyushkin, Phys. Lett. B 385, 333 (1996).

[13] A. V. Radyushkin, Phys. Rev. D 56, 5524 (1997).

[14] A. V. Radyushkin, Phys. Lett. B 380, 417 (1996).

[15] X. -D. Ji, J. Phys. G 24, 1181 (1998).

[16] D. Müller, D. Robaschik, B. Geyer, F. M. Dittes and J.Horejsi, Fortsch. Phys. 42, 101 (1994).

[17] M. Diehl, T. Gousset, B. Pire and O. Teryaev, Phys. Rev. Lett. 81, 1782 (1998).

[18] B. Pire and L. Szymanowski, Phys. Lett. B 556, 129 (2003).

[19] G. 't Hooft and M. J. G. Veltman, Nucl. Phys. B 50, 318 (1972). 
[20] G. 't Hooft and M. J. G. Veltman, NATO Adv. Study Inst. Ser. B Phys. 4, 177 (1974).

[21] G. 't Hooft and M. J. G. Veltman, Nucl. Phys. B 44, 189 (1972).

[22] D. J. Gross and F. Wilczek, Phys. Rev. Lett. 30, 1343 (1973).

[23] H. D. Politzer, Phys. Rept. 14, 129 (1974).

[24] J. Beringer et al. [Particle Data Group Collaboration], Phys. Rev. D 86, 010001 (2012).

[25] J. D. Bjorken and E. A. Paschos, Phys. Rev. 185, 1975 (1969).

[26] A. H. Mueller, in Advanced Series on Directions in High Energy Physics, 5 (World Scientific, Singapore, 1989), p 614.

[27] J. C. Collins, D. E. Soper and G. F. Sterman, Adv. Ser. Direct. High Energy Phys. 5, 1 (1988).

[28] C. G. Bollini and J. J. Giambiagi, Nuovo Cim. B 12, 20 (1972).

[29] V. N. Baier, E. A. Kuraev, V. S. Fadin and V. A. Khoze, Phys. Rept. 78, 293 (1981).

[30] B. Aubert et al. [BaBar Collaboration], Phys. Rev. D 80, 052002 (2009).

[31] A. V. Radyushkin, Phys. Rev. D 80, 094009 (2009).

[32] M. V. Polyakov, JETP Lett. 90, 228 (2009).

[33] E. Witten, Nucl. Phys. B 120, 189 (1977).

[34] S. Friot, B. Pire and L. Szymanowski, Phys. Lett. B 645, 153 (2007).

[35] M. Diehl, T. Gousset and B. Pire, Phys. Rev. D 62, 073014 (2000).

[36] I. V. Anikin, B. Pire and O. V. Teryaev, Phys. Rev. D 69, 014018 (2004).

[37] B. Pire, Annales Henri Poincare 4, S243 (2003).

[38] M. El Beiyad, B. Pire, L. Szymanowski and S. Wallon, Phys. Rev. D 78, 034009 (2008). 
[39] M. Gluck, E. Reya and A. Vogt, Z. Phys. C 48, 471 (1990).

[40] J. Blumlein and S. Kurth, Phys. Rev. D 60, 014018 (1999).

[41] W. Furmanski and R. Petronzio, Nucl. Phys. B 195, 237 (1982).

[42] R. Toldra, Comput. Phys. Commun. 143, 287 (2002).

[43] N. Cabibbo and R. Petronzio, Nucl. Phys. B 137, 395 (1978).

[44] R. D. Ball and S. Forte, Phys. Lett. B 335, 77 (1994).

[45] R. D. Ball and S. Forte, Phys. Lett. B 336, 77 (1994).

[46] A. V. Kotikov and G. Parente, Nucl. Phys. B 549, 242 (1999).

[47] L. Mankiewicz, A. Saalfeld and T. Weigl, Phys. Lett. B 393, 175 (1997).

[48] S. Forte, L. Garrido, J. I. Latorre and A. Piccione, JHEP 0205, 062 (2002).

[49] L. Del Debbio et al. [NNPDF Collaboration], JHEP 0503, 080 (2005).

[50] A. D. Martin and M. G. Ryskin, Phys. Rev. D 57, 6692 (1998).

[51] B. Pire, J. Soffer and O. Teryaev, Eur. Phys. J. C 8, 103 (1999).

[52] A. V. Radyushkin, Phys. Rev. D 59, 014030 (1999).

[53] J. C. Collins, L. Frankfurt and M. Strikman, Phys. Rev. D 56, 2982 (1997).

[54] S. J. Brodsky, L. Frankfurt, J. F. Gunion, A. H. Mueller and M. Strikman, Phys. Rev. D 50, 3134 (1994).

[55] A. V. Radyushkin and A. Tandogan, Phys. Rev. D 89, 074003 (2014).

[56] A. Tandogan and A. V. Radyushkin, Int. J. Mod. Phys. Conf. Ser. 25, 1460037 (2014).

[57] A. Tandogan and A. V. Radyushkin, Int. J. Mod. Phys. Conf. Ser. 04, 227 (2011). 


\section{APPENDIX A}

\section{ANALYTIC EXPRESSIONS}

A.1 $\Phi_{1,1}(x, \zeta)$

$$
\begin{aligned}
\Phi_{1,1}(x, \zeta) & \equiv \int_{0}^{1} V(x, y)\left[\Phi_{1,0}(y, \zeta)-\Phi_{1,0}(x, \zeta)\right] d y \\
& =\theta(0<x<\zeta<1 / 2)\left\{(3-4 \zeta) \frac{x}{2 \zeta}+\frac{1}{2 \zeta}[x \bar{x} \ln x+(1-x \bar{x}) \ln \bar{x}]\right. \\
& -\frac{\zeta-x}{\zeta}[\ln (\zeta-x)+\ln (\bar{\zeta}-x)]+\frac{\ln \zeta+\ln \bar{\zeta}(1-3 \zeta) / \zeta}{2(1-2 \zeta)} \bar{x} \\
& \left.-\frac{1-4 \zeta}{\zeta(1-2 \zeta)}[(x-\zeta) \ln (\zeta-x)+(\bar{\zeta}-x) \ln (\bar{\zeta}-x)]\right\}-\{x \rightarrow \bar{x}\} \\
& +\theta(\zeta<x<\bar{\zeta})\left\{\frac{1-2 x}{2(1-2 \zeta)}[-(1+4 \zeta)+\ln \bar{\zeta}(1-3 \zeta) / \zeta]\right. \\
& +\frac{1-4 \zeta}{2 \zeta(1-2 \zeta)}[(x-\zeta) \ln (x-\zeta)-(\bar{\zeta}-x) \ln (\bar{\zeta}-x)+\bar{x} \ln \bar{x}-x \ln x] \\
& \left.+\frac{2(x-\zeta)}{1-2 \zeta} \ln (x-\zeta)-\frac{2(\bar{\zeta}-x)}{1-2 \zeta} \ln (\bar{\zeta}-x)+\frac{x^{2}+\bar{x}^{2}}{2(1-2 \zeta)} \ln \left(\frac{\bar{x}}{x}\right)\right\}
\end{aligned}
$$


A.2 $\chi(x, \zeta)$

$$
\begin{aligned}
\chi(x, \zeta) \equiv & \left.\Psi_{1,1}(x, \zeta)=\int_{0}^{1} V(x, y)\left[\Phi_{1,0}(y, \zeta)-\Phi_{1,0}(x, \zeta)\right] d y-w(x, \zeta) \Phi_{0}(x, \zeta)\right] \\
& =\theta(0<x<\zeta<1 / 2)\left\{(1-4 \zeta) \frac{x}{2 \zeta}+\frac{1}{2 \zeta}[x \bar{x} \ln x+(1-x \bar{x}) \ln \bar{x}]\right. \\
& -\frac{\zeta-x}{\zeta}[\ln (\zeta-x)+\ln (\bar{\zeta}-x)]+\frac{\ln \zeta+\ln \bar{\zeta}(1-3 \zeta) / \zeta}{2(1-2 \zeta)} \bar{x} \\
& -\frac{1-4 \zeta}{\zeta(1-2 \zeta)}[(x-\zeta) \ln (\zeta-x)+(\bar{\zeta}-x) \ln (\bar{\zeta}-x)]-\frac{x}{\zeta} \ln (1-2 \zeta) \\
& \left.-\frac{x}{2 \zeta}[(1+\zeta) \ln \bar{\zeta}+(2-\zeta) \ln \zeta]\right\}-\{x \rightarrow \bar{x}\} \\
& +\theta(\zeta<x<\bar{\zeta})\left\{\frac{1-2 x}{2(1-2 \zeta)}[(1-4 \zeta)+\ln \bar{\zeta}(1-3 \zeta) / \zeta]\right. \\
& +\frac{1-4 \zeta}{2 \zeta(1-2 \zeta)}[(x-\zeta) \ln (x-\zeta)-(\bar{\zeta}-x) \ln (\bar{\zeta}-x)+\bar{x} \ln \bar{x}-x \ln x] \\
& +\frac{2(x-\zeta)}{1-2 \zeta} \ln (x-\zeta)-\frac{2(\bar{\zeta}-x)}{1-2 \zeta} \ln (\bar{\zeta}-x)+\frac{x^{2}+\bar{x}^{2}}{2(1-2 \zeta)} \ln \left(\frac{\bar{x}}{x}\right) \\
& \left.+\frac{1-2 x}{2(1-2 \zeta)}[2 \ln (1-2 \zeta)+(1+\zeta) \ln \bar{\zeta}+(2-\zeta) \ln \zeta]\right\}
\end{aligned}
$$




\title{
VITA
}

\section{PERSONAL DATA}

\author{
Name: Asli Tandogan \\ Address: Department of Physics 4600 Elkhorn Ave \\ Norfolk, VA 23529
}

\section{EDUCATION}

August, 2007-August, 2014: Ph.D. in Physics- Old Dominion University, Norfolk, VA

August, 2007- August, 2008: MS in Physics- Old Dominion University, Norfolk, VA

January, 2006-June, 2007: MS in Physics- Middle East Technical University, Ankara

September, 2002-January, 2006: B.Sc. in Physics- Middle East Technical University, Ankara

\section{PUBLICATIONS AND CONFERENCE NOTES}

- "Analytic evolution of singular distribution amplitudes in QCD", A. V. Radyushkin and A. Tandogan, Phys. Rev. D, 89:074003, Mar 2014

- "Studies of analytic evolution of Two-Photon Generalized Distribution Amplitude in QCD", A. Tandogan and A.V. Radyushkin, Int.J.Mod.Phys.Conf.Ser., $25: 1460037,2014$

- "Method of Analytic Evolution of Flat Distribution Amplitudes in QCD", A. Tandogan and A.V. Radyushkin, Int.J.Mod.Phys.Conf.Ser. 04 227-238, 2011 